\title{
Orthogonal Sulfation Strategy for Synthetic Heparan Sulfate Ligands
}

\author{
Supporting Information
}

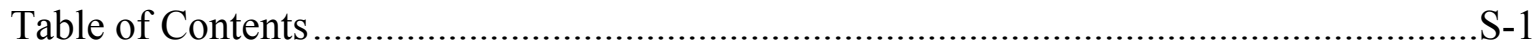

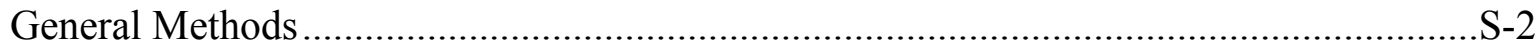

Experimental Procedures .......................................................................................

${ }^{1} \mathrm{H}$ NMR (300 MHz, $\mathrm{CDCl}_{3}$ ) Glucosamine Derivative 1............................................ 19

${ }^{13} \mathrm{C}$ NMR $\left(300 \mathrm{MHz}, \mathrm{CDCl}_{3}\right.$ ) Glucosamine Derivative 1............................................2-20

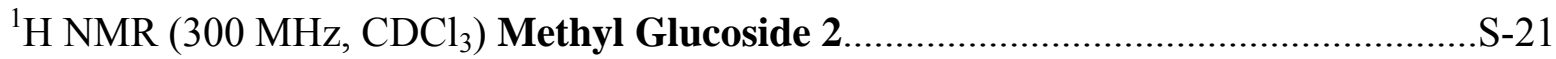

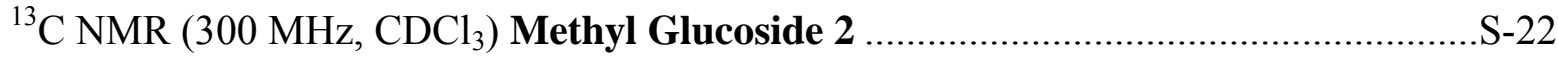

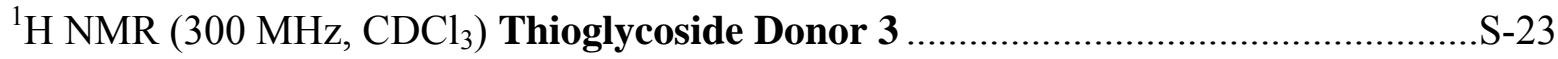

${ }^{13} \mathrm{C}$ NMR (500 MHz, $\mathrm{CDCl}_{3}$ ) Thioglycoside Donor 3 ….................................................2-24

${ }^{1} \mathrm{H}$ NMR $\left(300 \mathrm{MHz}, \mathrm{CDCl}_{3}\right.$ ) Bicyclic Lactone Acceptor 4 ..........................................2-25

${ }^{13} \mathrm{C}$ NMR $\left(500 \mathrm{MHz}, \mathrm{CDCl}_{3}\right)$ Bicyclic Lactone Acceptor 4 ……..................................

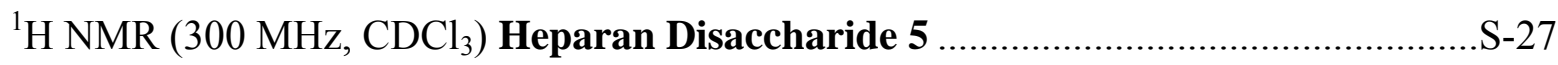

${ }^{13} \mathrm{C}$ NMR $\left(300 \mathrm{MHz}, \mathrm{CDCl}_{3}\right.$ ) Heparan Disaccharide 5 ................................................2-28

${ }^{1} \mathrm{H}$ NMR (300 MHz, $\mathrm{CDCl}_{3}$ ) Methyl Glucuronate 6 .................................................

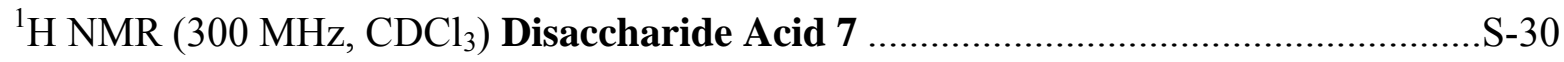

${ }^{1} \mathrm{H}$ NMR (300 MHz, $\mathrm{CDCl}_{3}$ ) Disaccharide Benzyl Ester 8 ............................................

${ }^{13} \mathrm{C}$ NMR $\left(500 \mathrm{MHz}, \mathrm{CDCl}_{3}\right.$ ) Disaccharide Benzyl Ester 8.......................................... -32

${ }^{1} \mathrm{H}$ NMR (300 MHz, 3:1 $\mathrm{CDCl}_{3}: \mathrm{CD}_{3} \mathrm{OD}$ ) Disaccharide 6'-O-Sulfate 9...........................

${ }^{13} \mathrm{C}$ NMR (500 MHz, $\mathrm{CD}_{3} \mathrm{OD}$ ) Disaccharide 6'-O-Sulfate 9 ............................................3

${ }^{1} \mathrm{H}$ NMR (300 MHz, 3:1 CDCl $3: \mathrm{CD}_{3} \mathrm{OD}$ ) Disaccharide 4'-O-Sulfate 10.........................S-35

${ }^{13} \mathrm{C}$ NMR (500 MHz, $\mathrm{CD}_{3} \mathrm{OD}$ ) Disaccharide 4'-O-Sulfate 10 …....................................36

${ }^{1} \mathrm{H}$ NMR (300 MHz, 3:1 $\mathrm{CDCl}_{3}: \mathrm{CD}_{3} \mathrm{OD}$ ) Disaccharide 3'-O-Sulfate 11 .........................

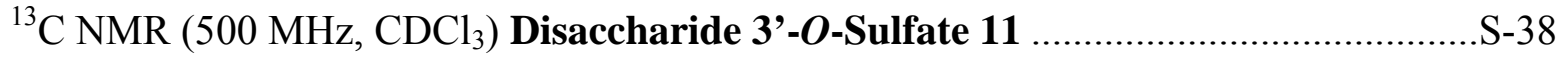

${ }^{1} \mathrm{H}$ NMR (300 MHz, 3:1 CDCl $3: \mathrm{CD}_{3} \mathrm{OD}$ ) Disaccharide 3-O-Sulfate 12 .........................

${ }^{13} \mathrm{C}$ NMR $\left(500 \mathrm{MHz}, \mathrm{CDCl}_{3}\right.$ ) Disaccharide 3-O-Sulfate 12 ...........................................

${ }^{1} \mathrm{H}$ NMR (300 MHz, 3:1 $\mathrm{CDCl}_{3}: \mathrm{CD}_{3} \mathrm{OD}$ ) Disaccharide 2-O-Sulfate 13 ......................... -41 


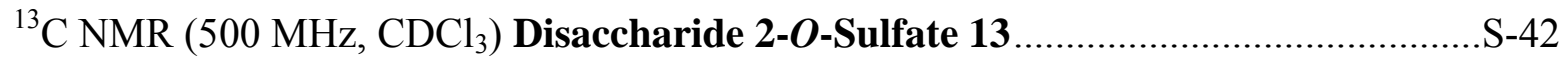

${ }^{1} \mathrm{H}$ NMR (300 MHz, 3:1 CDCl $: \mathrm{CD}_{3} \mathrm{OD}$ ) Disaccharide 6'-O-Sulfate-4'-OH 14 .............S-43

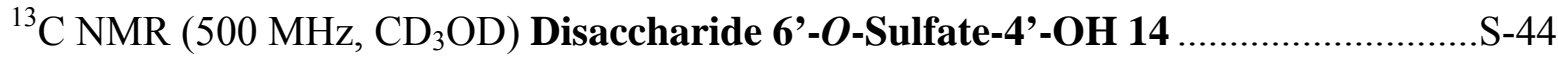

${ }^{1} \mathrm{H}$ NMR (300 MHz, 3:1 CDCl $3: \mathrm{CD}_{3} \mathrm{OD}$ ) Disaccharide 4'-O-Sulfate-3'-OH 15 .............S-45

${ }^{13} \mathrm{C}$ NMR (500 MHz, CD $\mathrm{CD}_{3} \mathrm{OD}$ ) Disaccharide 4'-O-Sulfate-3'-OH 15 ............................ -46

${ }^{1} \mathrm{H}$ NMR (300 MHz, 3:1 CDCl 3 : $\mathrm{CD}_{3} \mathrm{OD}$ ) Disaccharide 3'-O-Sulfate-2'-NHAc 16 .........S-47

${ }^{13} \mathrm{C}$ NMR (500 MHz, $\mathrm{CD}_{3} \mathrm{OD}$ ) Disaccharide 3'-O-Sulfate-2'-NHAc 16.........................

${ }^{1} \mathrm{H}$ NMR (300 MHz, 3:1 $\mathrm{CDCl}_{3}: \mathrm{CD}_{3} \mathrm{OD}$ ) Disaccharide 3-O-Sulfate-2-OH 17 ...............S-49

${ }^{13} \mathrm{C}$ NMR (500 MHz, $\mathrm{CD}_{3} \mathrm{OD}$ ) Disaccharide 3-O-Sulfate-2-OH 17 ..............................5-50

${ }^{1} \mathrm{H}$ NMR (300 MHz, 3:1 CDCl $3: \mathrm{CD}_{3} \mathrm{OD}$ ) Disaccharide 2-O-Sulfate-3-OH 18 ................S-51

${ }^{13} \mathrm{C}$ NMR (500 MHz, $\mathrm{CD}_{3} \mathrm{OD}$ ) Disaccharide 2-O-Sulfate-3-OH 18..............................S-52

${ }^{1} \mathrm{H}$ NMR (300 MHz, 3:1 CDCl 3 : $\mathrm{CD}_{3} \mathrm{OD}$ ) Disaccharide 2'-N-Sulfate-3'-OH 19 …..........S-53

${ }^{13} \mathrm{C}$ NMR (500 MHz, $\mathrm{CD}_{3} \mathrm{OD}$ ) Disaccharide 2'- $\mathrm{N}$-Sulfate-3'-OH 19............................

General Methods. All chemicals were obtained from Aldrich and used as received unless otherwise noted. IR spectra were acquired with a Nicolet Nexus 670 FT-IR spectrometer. Optical rotation were measured at $20-25{ }^{\circ} \mathrm{C}$ with a Rudolph Research AUTOPOL ${ }^{\circledR}$ III polarimeter. ${ }^{1} \mathrm{H}$ and ${ }^{13} \mathrm{C}$ spectra were recorded on a Varian Unity Inova NMR spectrometer operating at $300 \mathrm{MHz}$ and $75 \mathrm{MHz}$ respectively or a Bruker DRX 500 operating at $500 \mathrm{MHz}$ and $125 \mathrm{MHz}$ respectively, and referenced to the solvent used $\left(7.27 \mathrm{ppm}\right.$ and $77.23 \mathrm{ppm}$ for $\mathrm{CDCl}_{3}$, $3.31 \mathrm{ppm}$ and $49.15 \mathrm{ppm}$ for $\mathrm{CD}_{3} \mathrm{OD}$ ). Mass spectra were acquired using either a Hewlett Packard 5989B or a Finnigan 4000 mass spectrometer. The purity of the products was assessed using TLC on Silica Gel $60 \mathrm{~F}_{254}$ (Merck) with either ethanolic $p$-anisaldehyde or ninhydrin staining solutions. Chromatographic separations were performed using silica gel (ICN SiliTech 32-63D). 


\section{Experimental Procedures.}

Thiotolyl 2-azido-6-O-(tert-butyldiphenylsilyl)-2-deoxy-3-levulinoyl-4-O-(pmethoxybenzyl)- $\beta$-D-glucopyranoside (3). Thiotolyl 3-O-acetyl-2-deoxy-4,6-O-( $p$ methoxybenzylidene)-2-phthalimido $\beta$-D-glucopyranoside (1) (6.010 g, $10.400 \mathrm{mmol}$ ) was cooled to $-20{ }^{\circ} \mathrm{C}$, dissolved in $\mathrm{BH}_{3}$-THF complex (32 mL, $1 \mathrm{M}$ solution in THF), and treated with $\mathrm{Bu}_{2} \mathrm{BOTf}\left(11 \mathrm{~mL}, 1 \mathrm{M}\right.$ solution in $\left.\mathrm{CH}_{2} \mathrm{Cl}_{2}\right)$. The reaction mixture was stirred from -20 to $-5{ }^{\circ} \mathrm{C}$ over $1.5 \mathrm{~h}$ under argon, then quenched with $\mathrm{Et}_{3} \mathrm{~N}(5 \mathrm{~mL})$ followed by the dropwise addition of $\mathrm{MeOH}(50 \mathrm{~mL})$. The reaction mixture was warmed to $\mathrm{rt}$ and evaporated to dryness. The residual syrup was coevaporated with $\mathrm{MeOH}(2 \times 50 \mathrm{~mL})$ then toluene $(50 \mathrm{~mL})$. Purification by flash chromatography on silica gel (20-40\% EtOAc gradient in hexanes) afforded the intermediate C-6 alcohol (5.071 g, 84\% yield).

A solution of C-6 alcohol (3.670 g, $6.380 \mathrm{mmol})$ in anhydrous DMF (5 mL) was treated with tert-butylchlorodiphenylsilane $(1.99 \mathrm{~mL}, 7.660 \mathrm{mmol})$ and imidazole $(0.220 \mathrm{~g}, 3.20 \mathrm{mmol})$. The reaction mixture was stirred at $\mathrm{rt}$ for $24 \mathrm{~h}$ under argon, then diluted with $\mathrm{Et}_{2} \mathrm{O}(150 \mathrm{~mL})$ and quenched at $0{ }^{\circ} \mathrm{C}$ with sat. $\mathrm{NaHCO}_{3}$ solution $(100 \mathrm{~mL})$ and separated. The aqueous layer was extracted with additional $\mathrm{Et}_{2} \mathrm{O}(2 \times 75 \mathrm{~mL})$; the combined organic phases were then washed with water $(2 \times 75 \mathrm{~mL})$, brine $(100 \mathrm{~mL})$ and dried over $\mathrm{Na}_{2} \mathrm{SO}_{4}$. Purification by flash chromatography on silica gel (10-40\% EtOAc gradient in hexanes) afforded the intermediate 6-O-TBDPS ether as a white foam $(4.601 \mathrm{~g}, 88 \%$ yield $)$.

The C-6-TBDPS ether $(0.835 \mathrm{~g}, 1.020 \mathrm{mmol})$ was dissolved in $\mathrm{CH}_{2} \mathrm{Cl}_{2}(5 \mathrm{~mL})$ and treated with $\mathrm{NaOMe}(1 \mathrm{~mL}, 1 \mathrm{M}$ in $\mathrm{MeOH})$. The mixture was stirred at $\mathrm{rt}$ for $4 \mathrm{~h}$ under argon, then neutralized with activated Dowex 50X-W- $\left(\mathrm{H}^{+}\right)$ion exchange resin and filtered. The crude alcohol was then concentrated and dried under reduced pressure for $2 \mathrm{~h}$. The crude alcohol was dissolved in $n \mathrm{BuOH}(10 \mathrm{~mL})$ and treated with ethylenediamine $(1.7 \mathrm{~mL}, 25.5 \mathrm{mmol})$. The reaction mixture was stirred at $100{ }^{\circ} \mathrm{C}$ in a pressure vessel for $12 \mathrm{~h}$, then concentrated to dryness, coevaporated with toluene and dried under reduced pressure for $2 \mathrm{~h}$. The free amine was used without further purification.

Preparation of triflyl azide $\left(\mathrm{TfN}_{3}\right.$ ) solution in $\mathrm{CH}_{2} \mathrm{Cl}_{2}$ : (Caution: $\mathrm{TfN}_{3}$ is explosive when not in solution or when contact with acid) a solution of $\mathrm{NaN}_{3}(8.3 \mathrm{~g}, 128 \mathrm{mmol})$ in water $(21 \mathrm{~mL})$ was diluted with $\mathrm{CH}_{2} \mathrm{Cl}_{2}(35 \mathrm{~mL})$, cooled to $0{ }^{\circ} \mathrm{C}$, and treated with triflic anhydride $\left(\mathrm{Tf}_{2} \mathrm{O}, 4.3\right.$ 
$\mathrm{mL}, 25.5 \mathrm{mmol})$. The biphasic solution was stirred at $0{ }^{\circ} \mathrm{C}$ for $2.5 \mathrm{~h}$, diluted with water $(10 \mathrm{~mL})$, and extracted with $\mathrm{CH}_{2} \mathrm{Cl}_{2}(2 \times 10 \mathrm{~mL})$. The combined organic extracts where washed with saturated aq $\mathrm{Na}_{2} \mathrm{CO}_{3}$ solution $(10 \mathrm{~mL})$, dried over $\mathrm{Na}_{2} \mathrm{SO}_{4}$ and used immediately.

A solution of the crude amine, $\mathrm{CuSO}_{4} \cdot 5 \mathrm{H}_{2} \mathrm{O}(25.5 \mathrm{mg}, 0.10 \mathrm{mmol})$, and $\mathrm{K}_{2} \mathrm{CO}_{3}(212 \mathrm{mg}$, $1.5 \mathrm{mmol})$ in 9:1 MeOH- $\mathrm{H}_{2} \mathrm{O}(5 \mathrm{~mL})$ at $0{ }^{\circ} \mathrm{C}$ was treated with excess $\mathrm{TfN}_{3}(55 \mathrm{~mL}, \sim 0.46 \mathrm{M}$ solution in $\left.\mathrm{CH}_{2} \mathrm{Cl}_{2}\right)$. $\mathrm{MeOH}(1 \mathrm{~mL})$ was added to homogenize the mixture. The green reaction mixture was stirred at $\mathrm{rt}$ for $48 \mathrm{~h}$, then quenched at $0{ }^{\circ} \mathrm{C}$ with saturated aq $\mathrm{Na}_{2} \mathrm{CO}_{3}$ solution (50 $\mathrm{mL})$ and separated. The aqueous layer was extracted with additional $\mathrm{CH}_{2} \mathrm{Cl}_{2}(2 \times 100 \mathrm{~mL})$; the combined organic phases were then washed with water $(100 \mathrm{~mL})$, brine $(100 \mathrm{~mL})$ and dried over $\mathrm{Na}_{2} \mathrm{SO}_{4}$. Purification by flash chromatography on silica gel (10-30\% EtOAc gradient in hexanes) afforded the intermediate C-3 alcohol (0.600 g, 88\% yield over 3 steps).

A solution of DCC $(0.439 \mathrm{~g}, 2.130 \mathrm{mmol})$ and DMAP $(0.005 \mathrm{~g}, 0.043 \mathrm{mmol})$ in $\mathrm{CH}_{2} \mathrm{Cl}_{2}(1$ $\mathrm{mL})$ was treated with levulinic acid $(0.218 \mathrm{~mL}, 2.130 \mathrm{mmol})$ at $\mathrm{rt}$ and cooled to $0{ }^{\circ} \mathrm{C}$. After 5 min, a solution of C-3 alcohol $(0.200 \mathrm{~g}, 0.213 \mathrm{mmol})$ in $\mathrm{CH}_{2} \mathrm{Cl}_{2}(1 \mathrm{~mL})$ was added at $0{ }^{\circ} \mathrm{C}$. The reaction mixture was stirred at $\mathrm{rt}$ for $24 \mathrm{~h}$, then quenched at $0{ }^{\circ} \mathrm{C}$ with saturated aqueous $\mathrm{NaHCO}_{3}$ solution $(20 \mathrm{~mL})$ and separated. The aqueous layer was extracted with additional $\mathrm{CH}_{2} \mathrm{Cl}_{2}(2 \mathrm{x}$ $100 \mathrm{~mL})$; the combined organic phases were then washed with brine $(20 \mathrm{~mL})$ and dried over $\mathrm{Na}_{2} \mathrm{SO}_{4}$. Purification by flash chromatography on silica gel (10-30\% EtOAc gradient in hexanes) afforded compound $3(0.192 \mathrm{~g}, 90 \% \text { yield). [ } \alpha]_{\mathrm{D}}-56.0^{\circ}$ (c 2.0, $\left.\mathrm{CHCl}_{3}\right)$; IR (neat): 3071 , 2109, 1751, 1720, $1612 \mathrm{~cm}^{-1} ;{ }^{1} \mathrm{H}$ NMR $\left(\mathrm{CDCl}_{3}, 300 \mathrm{MHz}\right): \delta 7.80(2 \mathrm{H}, \mathrm{dd}, J=1.5,7.8 \mathrm{~Hz}), 7.74$ $(2 \mathrm{H}, \mathrm{dd}, J=1.5,7.8 \mathrm{~Hz}), 7.54(2 \mathrm{H}, \mathrm{d}, J=8.1 \mathrm{~Hz}), 7.36-7.46(6 \mathrm{H}, \mathrm{m}), 7.07(4 \mathrm{H}, \mathrm{d}, J=8.1 \mathrm{~Hz})$, $6.80(2 \mathrm{H}, \mathrm{d}, J=8.4 \mathrm{~Hz}), 5.15(1 \mathrm{H}, \mathrm{t}, J=9.6 \mathrm{~Hz}), 4.55(2 \mathrm{H}, \mathrm{AB}), 4.46(1 \mathrm{H}, \mathrm{d}, J=10.2 \mathrm{~Hz}), 4.01$ $(1 \mathrm{H}, \mathrm{d}, J=10.2 \mathrm{~Hz}), 3.92(1 \mathrm{H}, \mathrm{dd}, J=3.3,11.7 \mathrm{~Hz}), 3.79(3 \mathrm{H}, \mathrm{s}), 3.69(1 \mathrm{H}, \mathrm{t}, J=9.3 \mathrm{~Hz}), 3.34-$ $3.42(1 \mathrm{H}, \mathrm{m}), 3.34(1 \mathrm{H}, \mathrm{t}, J=9.9 \mathrm{~Hz}), 2.71-2.80(2 \mathrm{H}, \mathrm{m}), 2.54-2.59(2 \mathrm{H}, \mathrm{m}), 2.34(3 \mathrm{H}, \mathrm{s}), 2.19$ $(3 \mathrm{H}, \mathrm{s}), 1.14(9 \mathrm{H}, \mathrm{s}) ;{ }^{13} \mathrm{C} \mathrm{NMR}\left(\mathrm{CDCl}_{3}, 125 \mathrm{MHz}\right): \delta 206.47,172.24,159.83,139.09,136.36$, 136.14, 134.50, 133.84, 133.37, 130.45, 130.33, 130.27, 130.06, 128.34, 128.25, 127.94, 114.29, $86.61,80.54,77.25,75.52,74.86,63.81,62.87,55.73,38.25,30.27,28.48,27.45,27.45,21.68$, 19.82; ESIMS: $m / z$ 790.08, $[\mathrm{M}+\mathrm{Na}]^{+}$.

Methyl 2-O-acetyl- $\alpha$-D-3,6-glucuronolactone (4). Compound 2 (4.200 g, 11.864 mmol) was cooled to $0{ }^{\circ} \mathrm{C}$, dissolved in $\mathrm{BH}_{3}-\mathrm{THF}$ complex (36 mL, $1 \mathrm{M}$ solution in $\mathrm{THF}$ ), and treated with 
$\mathrm{Bu}_{2} \mathrm{BOTf}\left(13.050 \mathrm{~mL}, 1 \mathrm{M}\right.$ solution in $\left.\mathrm{CH}_{2} \mathrm{Cl}_{2}\right)$. The reaction mixture was stirred at $0{ }^{\circ} \mathrm{C}$ for $2 \mathrm{~h}$ under argon, then cooled to $-78^{\circ} \mathrm{C}$, quenched with $\mathrm{Et}_{3} \mathrm{~N}(10 \mathrm{~mL})$, followed by the dropwise addition of $\mathrm{MeOH}(100 \mathrm{~mL})$. The reaction mixture was warmed to $\mathrm{rt}$ and evaporated to dryness. The residual syrup was coevaporated with $\mathrm{MeOH}(2 \times 50 \mathrm{~mL})$ and toluene $(50 \mathrm{~mL})$, then purified by flash chromatography on silica gel (20-30\% EtOAc gradient in hexanes) to afford the intermediate C-6 alcohol (3.650 g, 87\% yield).

A solution of C-6 alcohol $(3.440 \mathrm{~g}, 9.663 \mathrm{mmol})$ in $\mathrm{CH}_{2} \mathrm{Cl}_{2}(100 \mathrm{~mL})$ was cooled to $0{ }^{\circ} \mathrm{C}$. TEMPO (0.075 g, $0.483 \mathrm{mmol}), \mathrm{NaOCl}(100 \mathrm{~mL}, 5 \%$ aq solution) and saturated aqueous $\mathrm{NaHCO}_{3}$ solution $(100 \mathrm{~mL})$ were added at $0{ }^{\circ} \mathrm{C}$. After stirring at $0{ }^{\circ} \mathrm{C}$ for $30 \mathrm{~min}$, the reaction mixture was quenched with saturated aqueous $\mathrm{NH}_{4} \mathrm{Cl}(100 \mathrm{~mL})$. The mixture was brought to $\mathrm{pH}$ 3 with $4 \%$ aqueous $\mathrm{HCl}$, and the aqueous layer was extracted with EtOAc $(3 \times 500 \mathrm{~mL})$. The combined organic phases were dried over $\mathrm{Na}_{2} \mathrm{SO}_{4}$ and concentrated to dryness. The free acid $(3.540 \mathrm{~g})$ was used without further purification.

The crude free acid $(0.800 \mathrm{~g}, 2.162 \mathrm{mmol})$ was dissolved in $\mathrm{MeOH}(50 \mathrm{~mL})$ and treated with $\mathrm{K}_{2} \mathrm{CO}_{3}(0.895 \mathrm{~g}, 6.486 \mathrm{mmol})$. The mixture was stirred at $\mathrm{rt}$ for $24 \mathrm{~h}$ then concentrated to dryness, coevaporated with toluene, and dried under reduced pressure for $2 \mathrm{~h}$. The crude product was treated with $\mathrm{Ac}_{2} \mathrm{O}(50 \mathrm{~mL})$ and stirred at $75^{\circ} \mathrm{C}$ for $4 \mathrm{~h}$, then concentrated to dryness. Purification by flash chromatography on silica gel (10-30\% EtOAc gradient in hexanes) afforded the bicyclic [3.2.1] glucuronolactone ( $0.457 \mathrm{~g}, 60 \%$ yield over 3 steps).

A solution of methyl 2-O-acetyl-4-( $p$-methoxybenzyl)- $\alpha$-D-3,6-glucuronolactone $(0.050 \mathrm{~g}$, $0.142 \mathrm{mmol})$ in $90 \%$ aqueous $\mathrm{CH}_{3} \mathrm{CN}(2 \mathrm{~mL})$ was treated with $\mathrm{CAN}(0.234 \mathrm{~g}, 0.426 \mathrm{mmol})$ at 0 ${ }^{\circ} \mathrm{C}$. The reaction mixture was stirred at $0{ }^{\circ} \mathrm{C}$ for $12 \mathrm{~h}$, then quenched with saturated aqueous $\mathrm{Na}_{2} \mathrm{~S}_{2} \mathrm{O}_{3}(5 \mathrm{~mL})$, extracted with $\mathrm{CH}_{2} \mathrm{Cl}_{2}(3 \times 20 \mathrm{~mL})$, dried over $\mathrm{Na}_{2} \mathrm{SO}_{4}$, and concentrated to dryness. Purification by flash chromatography on silica gel (20-30\% EtOAc gradient in hexanes) afforded compound $4(0.023 \mathrm{~g}, 65 \%$ yield $)$. $[\alpha]_{\mathrm{D}}+39.5^{\circ}$ (c 1.2, $\left.\mathrm{CHCl}_{3}\right)$; IR (neat): 3544,1806 , $1748 \mathrm{~cm}^{-1} ;{ }^{1} \mathrm{H} \mathrm{NMR}\left(\mathrm{CDCl}_{3}, 300 \mathrm{MHz}\right): \delta 5.40(1 \mathrm{H}, \mathrm{t}, J=3.0 \mathrm{~Hz}), 5.04(1 \mathrm{H}, \mathrm{d}, J=3.6 \mathrm{~Hz})$, $4.84(1 \mathrm{H}, \mathrm{t}, J=3.0 \mathrm{~Hz}), 4.34(1 \mathrm{H}, \mathrm{s}), 4.30(1 \mathrm{H}, \mathrm{dt}, J=3.0,12.0 \mathrm{~Hz}), 3.99(1 \mathrm{H}, \mathrm{d}, J=12.3 \mathrm{~Hz})$, $3.65(3 \mathrm{H}, \mathrm{s}), 2.25(3 \mathrm{H}, \mathrm{s}) ;{ }^{13} \mathrm{C} \mathrm{NMR}\left(\mathrm{CDCl}_{3}, 125 \mathrm{MHz}\right): \delta 169.97,169.36,97.27,75.01,72.25$, 69.66, 67.80, 58.23, 20.99; ESIMS: $m / z$ 249.12, [M+H] ${ }^{+}$. 


\section{Methyl (methyl 4-O-[2-azido-6-O-(tert-butyldiphenylsilyl)-2-deoxy-3-O-levulinoyl-4-O-(p-} methoxybenzyl)- $\alpha$-D-glucopyranosyl]-2-O-acetyl-3-O-[2-(trimethylsilyl)ethoxymethyl]- $\alpha$-Dgluco-pyranosyluronate) (5). Benzenesulfinyl piperidine (BSP) was prepared according to the method described by Crich and Smith. ${ }^{19}$ A reaction mixture containing freshly prepared BSP (0.078 g, $0.372 \mathrm{mmol})$, DTBMP (0.077 g, $0.372 \mathrm{mmol})$, and activated 4A powdered sieves in dry $\mathrm{CH}_{2} \mathrm{Cl}_{2}(1 \mathrm{~mL})$ was stirred at $\mathrm{rt}$ under an argon atmosphere for $1 \mathrm{~h}$. The solution was cooled to $-60{ }^{\circ} \mathrm{C}$ and treated with $\mathrm{Tf}_{2} \mathrm{O}(0.063 \mathrm{~mL}, 0.372 \mathrm{mmol})$, then stirred for $30 \mathrm{~min}$. A precooled solution of glycosyl donor $3(0.220 \mathrm{~g}, 0.286 \mathrm{mmol})$ in dry $\mathrm{CH}_{2} \mathrm{Cl}_{2}(1 \mathrm{~mL})$ was added at $-60{ }^{\circ} \mathrm{C}$ and the reaction mixture was stirred for $30 \mathrm{~min}$. A precooled solution of glycosyl acceptor 4 $(0.133 \mathrm{~g}, 0.572 \mathrm{mmol})$ in dry $\mathrm{CH}_{2} \mathrm{Cl}_{2}(1 \mathrm{~mL})$ was transferred by cannula to the reaction mixture which was stirred at $-60{ }^{\circ} \mathrm{C}$ for an additional $18 \mathrm{~h}$, then slowly warmed to rt over a 2-h period. The reaction mixture was then treated with methanol $(10 \mathrm{~mL})$, filtered, and concentrated under reduced pressure.

The crude reaction mixture was redissolved in methanol $(10 \mathrm{~mL})$ and treated with $\mathrm{NaOAc}$ $(0.023 \mathrm{~g}, 0.286 \mathrm{mmol})$, then stirred at $\mathrm{rt}$ for $3 \mathrm{~h}$. The reaction was quenched with saturated aqueous $\mathrm{NH}_{4} \mathrm{Cl}$, extracted with dichloromethane $(3 \times 50 \mathrm{~mL})$, dried over $\mathrm{Na}_{2} \mathrm{SO}_{4}$, then concentrated under reduced pressure. Purification by silica gel chromatography $(30 \%$ EtOAc in hexanes) yielded the 3-OH disaccharide methyl ester as a clear viscous oil $(0.166 \mathrm{~g}, 64 \%)$. $[\alpha]_{\mathrm{D}}$ $+127.2^{\circ}$ (c 2.0, $\mathrm{CHCl}_{3}$ ); IR (neat): 3482.9, 2111.5, 1749.4, $1720.9 \mathrm{~cm}^{-1} ;{ }^{1} \mathrm{H} \mathrm{NMR}\left(\mathrm{CDCl}_{3}, 300\right.$ MHz): $\delta 7.69(4 \mathrm{H}, \mathrm{t}, J=8.1 \mathrm{~Hz}), 7.36-7.45(6 \mathrm{H}, \mathrm{m}), 7.13(2 \mathrm{H}, \mathrm{d}, J=8.7 \mathrm{~Hz}), 6.83(2 \mathrm{H}, \mathrm{d}, J=$ $8.7 \mathrm{~Hz}), 5.52(1 \mathrm{H}, \mathrm{t}, J=9.9 \mathrm{~Hz}), 5.25(1 \mathrm{H}, \mathrm{d}, J=3.3 \mathrm{~Hz}), 4.91(1 \mathrm{H}, \mathrm{d}, J=3.3 \mathrm{~Hz}), 4.89(1 \mathrm{H}, \mathrm{dd}$, $J=3.6,10.2 \mathrm{~Hz}), 4.62(2 \mathrm{H}, \mathrm{s}), 4.00-4.14(3 \mathrm{H}, \mathrm{m}), 3.93$ (1H, t, $J=9.6 \mathrm{~Hz}), 3.77-3.84(2 \mathrm{H}, \mathrm{m})$, $3.80(3 \mathrm{H}, \mathrm{s}), 3.64-3.70(2 \mathrm{H}, \mathrm{m}), 3.51(1 \mathrm{H}, \mathrm{dd}, J=3.3,10.5 \mathrm{~Hz}), 3.37(6 \mathrm{H}, \mathrm{s}), 2.72-2.78(2 \mathrm{H}$, m), 2.54-2.60 (2H, m), $2.20(3 \mathrm{H}, \mathrm{s}), 2.15(3 \mathrm{H}, \mathrm{s}), 1.11(9 \mathrm{H}, \mathrm{s}) ;{ }^{13} \mathrm{C} \mathrm{NMR}\left(\mathrm{CDCl}_{3}, 125 \mathrm{MHz}\right): \delta$ 206.53, 172.31, 171.07, 168.98, 159.68, 136.37, 136.08, 133.87, 133.42, 130.68, 130.24, 129.78, 128.24, 128.10, 114.20, 99.93, 97.73, 81.92, 75.75, 74.89, 73.86, 72.91, 72.48, 71.38, 70.38, 63.52, 62.30, 56.17, 55.72, 52.98, 38.18, 30.30, 28.42, 27.43, 21.35, 19.87; ESIMS: m/z 930.19, $[\mathrm{M}+\mathrm{Na}]^{+}$.

A solution of $3-\mathrm{OH}$ disaccharide methyl ester $(0.167 \mathrm{~g}, 0.184 \mathrm{mmol})$ in dry $\mathrm{CH}_{2} \mathrm{Cl}_{2}(4 \mathrm{~mL})$ was treated with $(i \operatorname{Pr})_{2} \mathrm{NEt}(0.170 \mathrm{~mL}, 1.840 \mathrm{mmol})$, SEM-Cl $(0.228 \mathrm{~mL}, 1.289 \mathrm{mmol})$, and $(n \mathrm{Bu})_{4} \mathrm{NI}(0.102 \mathrm{~g}, 0.276 \mathrm{mmol})$. The reaction mixture was stirred at $\mathrm{rt}$ for $48 \mathrm{~h}$, diluted with 
dichloromethane $(50 \mathrm{~mL})$, quenched at $0{ }^{\circ} \mathrm{C}$ with saturated aqueous $\mathrm{NH}_{4} \mathrm{Cl}$ solution $(20 \mathrm{~mL})$, extracted with $\mathrm{CH}_{2} \mathrm{Cl}_{2}(3 \times 50 \mathrm{~mL})$, dried over $\mathrm{Na}_{2} \mathrm{SO}_{4}$, then concentrated under reduced pressure. Purification by silica gel chromatography (10\% EtOAc in hexanes) yielded compound 5 as a clear viscous oil $(0.141 \mathrm{~g}, 74 \%)$. $[\alpha]_{\mathrm{D}}+131.1^{\circ}$ (c 1.5, $\left.\mathrm{CHCl}_{3}\right)$; IR (neat): 2109.1, 1751.1, 1721.0, 1612.9 $\mathrm{cm}^{-1} ;{ }^{1} \mathrm{H}$ NMR ( $\left.\mathrm{CDCl}_{3}, 300 \mathrm{MHz}\right): \delta 7.67-7.72(4 \mathrm{H}, \mathrm{m}), 7.37-7.44(6 \mathrm{H}, \mathrm{m}), 7.12$ $(2 \mathrm{H}, \mathrm{d}, J=8.7 \mathrm{~Hz}), 6.81(2 \mathrm{H}, \mathrm{d}, J=8.7 \mathrm{~Hz}), 5.56(1 \mathrm{H}, \mathrm{d}, J=3.3 \mathrm{~Hz}), 5.46(1 \mathrm{H}, \mathrm{t}, J=9.6 \mathrm{~Hz})$, 4.81-4.92 (4H, m), $4.61(2 \mathrm{H}, \mathrm{AB}), 4.23(1 \mathrm{H}, \mathrm{d}, J=8.7 \mathrm{~Hz}), 3.84-4.11(5 \mathrm{H}, \mathrm{m}), 3.78(3 \mathrm{H}, \mathrm{s})$, 3.66-3.76 (1H, m), 3.49-3.61 (2H, m), $3.53(3 \mathrm{H}, \mathrm{s}), 3.38(3 \mathrm{H}, \mathrm{s}), 3.16(1 \mathrm{H}, \mathrm{dd}, J=3.6,11.1$ Hz), 2.72-2.77 (2H, m), 2.56-2.61 (2H, m), 2.18 (3H, s), 2.14 (3H, s), 1.10 (9H, s), 0.94 (2H, t, J $=8.4 \mathrm{~Hz}), 0.03(9 \mathrm{H}, \mathrm{s}) ;{ }^{13} \mathrm{C} \mathrm{NMR}\left(\mathrm{CDCl}_{3}, 125 \mathrm{MHz}\right): \delta 206.10,171.88,170.29,169.41,159.24$, $135.95,135.67,133.65,133.07,130.38,129.78,129.30,127.80,127.68,113.77,98.14,97.20$, 96.93, 78.68, 75.45, 75.15, 74.42, 72.40, 72.18, 71.93, 70.01, 66.63, 61.86, 55.77, 55.28, 52.66, $37.87,29.85,28.05,27.02,21.21,19.46,18.10,-1.34$; ESIMS: $m / z$ 1060.15, $\left[\mathrm{M}+\mathrm{Na}^{+}\right.$.

\section{Benzyl (methyl 4-O-[2-azido-6-O-(tert-butyldiphenylsilyl)-2-deoxy-3-O-levulinoyl-4-O-(p- methoxybenzyl)- $\alpha$-D-glucopyranosyl]-2-O-acetyl-3-O-[2-(trimethylsilyl)ethoxymethyl]- $\alpha$-D-} glucopyranosyluronate) (8). A reaction mixture containing BSP (0.036 g, $0.170 \mathrm{mmol})$, DTBMP (0.035 g, $0.170 \mathrm{mmol})$, and activated 4A powdered sieves in dry $\mathrm{CH}_{2} \mathrm{Cl}_{2}(1 \mathrm{~mL})$ was stirred at $\mathrm{rt}$ under an argon atmosphere for $1 \mathrm{~h}$. The solution was cooled to $-60{ }^{\circ} \mathrm{C}$ and treated with $\mathrm{Tf}_{2} \mathrm{O}(0.029 \mathrm{~mL}, 0.170 \mathrm{mmol})$, then stirred for $30 \mathrm{~min}$. A precooled solution of glycosyl donor $3(0.104 \mathrm{~g}, 0.131 \mathrm{mmol})$ in dry $\mathrm{CH}_{2} \mathrm{Cl}_{2}(1 \mathrm{~mL})$ was added at $-60{ }^{\circ} \mathrm{C}$, then stirred for 30 min. A precooled solution of glycosyl acceptor $4(0.046 \mathrm{~g}, 0.191 \mathrm{mmol})$ in dry $\mathrm{CH}_{2} \mathrm{Cl}_{2}(1 \mathrm{~mL})$ was transferred by cannula to the reaction mixture, which was stirred at $-60{ }^{\circ} \mathrm{C}$ for an additional $18 \mathrm{~h}$, then slowly warmed to rt over a $2-\mathrm{h}$ period. The reaction was quenched at $\mathrm{rt}$ with saturated aqueous $\mathrm{NaHCO}_{3}$ solution $(20 \mathrm{~mL})$, extracted with $\mathrm{CH}_{2} \mathrm{Cl}_{2}(3 \times 100 \mathrm{~mL})$, dried over $\mathrm{Na}_{2} \mathrm{SO}_{4}$, then concentrated under reduced pressure.

The crude reaction mixture was dissolved in $\mathrm{CH}_{2} \mathrm{Cl}_{2}(5 \mathrm{~mL})$ and hexanes $(5 \mathrm{~mL})$, treated with silica gel $(5.0 \mathrm{~g})$, then stirred at $\mathrm{rt}$ for $1 \mathrm{~h}$. The mixture was concentrated to dryness under reduced pressure, then redissolved in $\mathrm{CH}_{2} \mathrm{Cl}_{2}(5 \mathrm{~mL})$ and hexanes $(5 \mathrm{~mL})$, stirred at $\mathrm{rt}$ for $30 \mathrm{~min}$, and concentrated again under reduced pressure. This was repeated several times until TLC 
indicated that the lactone was no longer present. Purification by silica gel chromatography [3\% $\mathrm{MeOH}$ in $\mathrm{CH}_{2} \mathrm{Cl}_{2}$ ] yielded disaccharide acid 7 as a clear viscous oil $(0.074 \mathrm{~g})$.

A solution of disaccharide acid $7(0.074 \mathrm{~g}, 0.083 \mathrm{mmol})$ in $\mathrm{CH}_{2} \mathrm{Cl}_{2}(1 \mathrm{~mL})$ was treated with Oct $4 \mathrm{NBr}(0.045 \mathrm{~g}, 0.083 \mathrm{mmol}), \mathrm{BnBr}(0.079 \mathrm{~mL}, 0.664 \mathrm{mmol})$, and saturated aqueous $\mathrm{NaHCO}_{3}$ solution $(0.25 \mathrm{~mL})$. The reaction mixture was stirred at $\mathrm{rt}$ for $20 \mathrm{~h}$, diluted with $\mathrm{CH}_{2} \mathrm{Cl}_{2}(20 \mathrm{~mL})$ and water $(10 \mathrm{~mL})$, extracted with $\mathrm{CH}_{2} \mathrm{Cl}_{2}(3 \times 50 \mathrm{~mL})$ and dried over $\mathrm{Na}_{2} \mathrm{SO}_{4}$, then concentrated under reduced pressure. Purification by silica gel chromatography (25\% EtOAc in hexanes) yielded benzyl ester 8 as a clear viscous oil $\left(0.059 \mathrm{~g}, 42 \%\right.$ over three steps). $[\alpha]_{\mathrm{D}}+50.6^{\circ}$ (c 2.5 , $\left.\mathrm{CHCl}_{3}\right)$; IR (neat): $3480.1,2111.5,1748.5,1721.6 \mathrm{~cm}^{-1} ;{ }^{1} \mathrm{H} \mathrm{NMR}\left(\mathrm{CDCl}_{3}, 300 \mathrm{MHz}\right): \delta 7.69$ (4H, m), 7.36-7.45 (6H, m), 7.20-7.27 (3H, m), 7.07-7.13 (4H, m), $6.81(2 \mathrm{H}, \mathrm{d}, J=8.7 \mathrm{~Hz})$, $5.53(1 \mathrm{H}, \mathrm{t}, J=9.9 \mathrm{~Hz}), 5.25(1 \mathrm{H}, \mathrm{d}, J=3.3 \mathrm{~Hz}), 4.91(1 \mathrm{H}, \mathrm{d}, J=3.3 \mathrm{~Hz}), 4.75-4.81(2 \mathrm{H}, \mathrm{m})$, $4.60(1 \mathrm{H}, \mathrm{d}, J=10.5 \mathrm{~Hz}), 4.56(1 \mathrm{H}, \mathrm{d}, J=10.5 \mathrm{~Hz}), 4.09-4.17(2 \mathrm{H}, \mathrm{m}), 3.80-3.97(4 \mathrm{H}, \mathrm{m}), 3.79$ $(3 \mathrm{H}, \mathrm{s}), 3.68-3.78(2 \mathrm{H}, \mathrm{m}), 3.54(1 \mathrm{H}, \mathrm{dd}, J=3.3,10.5 \mathrm{~Hz}), 3.36(3 \mathrm{H}, \mathrm{s}), 2.72-2.80(2 \mathrm{H}, \mathrm{m})$, 2.55-2.60 (2H, m), $2.20(3 \mathrm{H}, \mathrm{s}), 2.14(3 \mathrm{H}, \mathrm{s}), 1.10(9 \mathrm{H}, \mathrm{s}) ;{ }^{13} \mathrm{C} \mathrm{NMR}\left(\mathrm{CDCl}_{3}, 125 \mathrm{MHz}\right)$ : $\delta 206.55,172.28,171.08,168.42,159.67,136.33,136.11,135.31,133.81,133.42,130.68$, $130.25,129.79,128.95,128.70,128.48,128.25,128.16,114.19,99.91,97.71,81.84,75.73$, $74.85,73.95,72.92,72.51,71.26,70.54,67.72,63.57,62.34,56.14,55.73,38.19,30.30,28.45$, 27.44, 21.35, 19.87; ESIMS: $m / z$ 1006.16, $[\mathrm{M}+\mathrm{Na}]^{+}$.

\section{Methyl (methyl 4-O-[2-azido-2-deoxy-3-O-levulinoyl-4-O-(p-methoxybenzyl)- 6-O-} sulfonato- $\alpha$-D-glucopyranosyl]-2- $O$-acetyl-3- $O$-[2-(trimethylsilyl)ethoxymethyl]- $\alpha-D-$ glucopyranosyluronate) sodium salt (9). A $1 \mathrm{M}$ solution of TBAF in THF ( $5 \mathrm{~mL})$ was brought to $\mathrm{pH} 9$ with HF.pyridine $(0.25 \mathrm{~mL})$ in a FEP tube. The resulting TBAF/HF.pyridine solution was stored at $-5^{\circ} \mathrm{C}$ until used. A solution of $5(0.051 \mathrm{~g}, 0.049 \mathrm{mmol})$ in THF $(2 \mathrm{~mL})$ was treated with the TBAF/HF.Pyridine solution $(1.470 \mathrm{~mL}, 1.470 \mathrm{mmol})$ at $0{ }^{\circ} \mathrm{C}$ in a FEP tube. The reaction mixture was vigorously stirred at $\mathrm{rt}$ for $40 \mathrm{~h}$, quenched at $0{ }^{\circ} \mathrm{C}$ with saturated aqueous $\mathrm{NaHCO}_{3}$ solution $(20 \mathrm{~mL})$, extracted with $\mathrm{CH}_{2} \mathrm{Cl}_{2}(3 \times 50 \mathrm{~mL})$, dried over $\mathrm{Na}_{2} \mathrm{SO}_{4}$, then concentrated under reduced pressure. Purification by silica gel chromatography (50\% EtOAc in hexanes) yielded the 6'-OH disaccharide as a clear viscous oil $(0.031 \mathrm{~g}, 80 \%)$. $[\alpha]_{\mathrm{D}}+123.9^{\circ}(\mathrm{c}$ 2.0, $\mathrm{CHCl}_{3}$ ); IR (neat): 2108.3, 1747.0, $1721.1 \mathrm{~cm}^{-1} ;{ }^{1} \mathrm{H}$ NMR $\left(\mathrm{CDCl}_{3}, 300 \mathrm{MHz}\right): \delta 7.22(2 \mathrm{H}, \mathrm{d}$, $J=8.7 \mathrm{~Hz}), 6.86(2 \mathrm{H}, \mathrm{d}, J=8.4 \mathrm{~Hz}), 5.55(1 \mathrm{H}, \mathrm{d}, J=3.6 \mathrm{~Hz}), 5.41(1 \mathrm{H}, \mathrm{t}, J=9.3 \mathrm{~Hz}), 4.76-$ 
$4.88(4 \mathrm{H}, \mathrm{m}), 4.57(2 \mathrm{H}, \mathrm{AB}), 4.19-4.22(1 \mathrm{H}, \mathrm{m}), 4.06-4.10(2 \mathrm{H}, \mathrm{m}), 3.74-3.80(1 \mathrm{H}, \mathrm{m}), 3.79$ $(3 \mathrm{H}, \mathrm{s}), 3.76(3 \mathrm{H}, \mathrm{s}), 3.46-3.70(6 \mathrm{H}, \mathrm{m}), 3.39(3 \mathrm{H}, \mathrm{s}), 3.13\left(1 \mathrm{H}, \mathrm{dd}, J=3.3,10.5 \mathrm{H}_{\mathrm{Z}}\right), 2.70-2.79$ $(2 \mathrm{H}, \mathrm{m}), 2.54-2.59(2 \mathrm{H}, \mathrm{m}), 2.17(3 \mathrm{H}, \mathrm{s}), 2.11(3 \mathrm{H}, \mathrm{s}), 0.89(2 \mathrm{H}, \mathrm{t}, J=8.4 \mathrm{~Hz}),-0.01(9 \mathrm{H}, \mathrm{s})$; ${ }^{13} \mathrm{C} \mathrm{NMR}\left(\mathrm{CDCl}_{3}, 125 \mathrm{MHz}\right): \delta 206.56,172.27,170.69,170.07,159.85,130.32,130.13,114.28$, 98.50, 97.75, 97.37, 79.16, 75.88, 75.51, 74.73, 72.70, 72.40, 72.18, 70.07, 66.99, 62.04, 61.58, 56.19, 55.71, 53.35, 38.23, 30.26, 28.41, 21.61, 18.47, -0.98; ESIMS: m/z 822.05, [M+ Na] ${ }^{+}$

A solution of 6'-OH disaccharide $(0.034 \mathrm{~g}, 0.042 \mathrm{mmol})$ in anhydrous pyridine $(1 \mathrm{~mL})$ was treated with $\mathrm{SO}_{3} \cdot \mathrm{NMe}_{3}$ complex $(0.112 \mathrm{~g}, 0.840 \mathrm{mmol})$. The reaction mixture was stirred at 55 ${ }^{\circ} \mathrm{C}$ in a pressure tube for $20 \mathrm{~h}$, then cooled to $0{ }^{\circ} \mathrm{C}$ and quenched with anhydrous methanol $(5$ $\mathrm{mL}$ ). The mixture was stirred at $\mathrm{rt}$ for $1 \mathrm{~h}$, and treated with a 1:1 mixture of saturated aqueous $\mathrm{NaHCO}_{3}$ solution and brine $(20 \mathrm{~mL})$, stirred for $1 \mathrm{~h}$, extracted with $\mathrm{CH}_{2} \mathrm{Cl}_{2}(4 \times 50 \mathrm{~mL})$, dried over $\mathrm{Na}_{2} \mathrm{SO}_{4}$, then concentrated under reduced pressure. Purification by silica gel chromatography $\left(5 \% \mathrm{MeOH}\right.$ in $\left.\mathrm{CH}_{2} \mathrm{Cl}_{2}\right)$ yielded disaccharide 6'-O-sulfate 9 as an amorphous white solid $(0.037 \mathrm{~g}, 96 \%)$. [ $\alpha]_{\mathrm{D}}+112.2^{\circ}\left(\mathrm{c} 2.0, \mathrm{CHCl}_{3}\right)$; IR (neat): 2109.5, 1748.2, 1722.1, 1515.2, $1248.4 \mathrm{~cm}^{-1} ;{ }^{1} \mathrm{H}$ NMR $\left(25 \% \mathrm{CD}_{3} \mathrm{OD} / \mathrm{CDCl}_{3}, 300 \mathrm{MHz}\right): \delta 7.17(2 \mathrm{H}, \mathrm{d}, J=8.7 \mathrm{~Hz}), 6.76$ $(2 \mathrm{H}, \mathrm{d}, J=8.4 \mathrm{~Hz}), 5.40(1 \mathrm{H}, \mathrm{d}, J=3.6 \mathrm{~Hz}), 5.29(1 \mathrm{H}, \mathrm{dd}, J=8.7,9.0 \mathrm{~Hz}), 4.68-4.81(4 \mathrm{H}, \mathrm{m})$, $4.58(1 \mathrm{H}, \mathrm{d}, J=10.5 \mathrm{~Hz}), 4.47(1 \mathrm{H}, \mathrm{d}, J=10.8 \mathrm{~Hz}), 4.21-4.24(1 \mathrm{H}, \mathrm{m}), 4.10-4.15(1 \mathrm{H}, \mathrm{m})$, 3.94-4.01 (2H, m), $3.70(3 \mathrm{H}, \mathrm{s}), 3.68(3 \mathrm{H}, \mathrm{s}), 3.36-3.64(5 \mathrm{H}, \mathrm{m}), 3.31(3 \mathrm{H}, \mathrm{s}), 3.13(1 \mathrm{H}, \mathrm{dd}, J=$ 3.6, $10.8 \mathrm{~Hz}), 2.63-2.69(2 \mathrm{H}, \mathrm{m}), 2.43-2.48(2 \mathrm{H}, \mathrm{m}), 2.11(3 \mathrm{H}, \mathrm{s}), 2.05(3 \mathrm{H}, \mathrm{s}), 0.82(2 \mathrm{H}, \mathrm{t}, J=$

$8.7 \mathrm{~Hz}),-0.08(9 \mathrm{H}, \mathrm{s}) ;{ }^{13} \mathrm{C} \mathrm{NMR}\left(\mathrm{CD}_{3} \mathrm{OD}, 125 \mathrm{MHz}\right): \delta 207.97,172.62,170.92,169.92,159.91$, $130.67,130.34,113.68,98.75,97.45,96.94,77.65,76.86,75.16,74.50,72.54,71.81,70.75$, 66.56, 66.52, 65.41, 61.91, 55.27, 54.71, 54.66, 52.52, 52.47, 37.46, 28.75, 28.03, 20.20, 17.93, -2.27; ESIMS: $m / z$ 924.84, [M+ Na $]^{+}, 878.04,[\mathrm{M}-\mathrm{Na}]^{-}$.

\section{Methyl (methyl 4-O-[2-azido-6-O-(tert-butyldiphenylsilyl)-2-deoxy-3-O-levulinoyl-4-O-} sulfonato- $\alpha$-D-glucopyranosyl]-2-O-acetyl-3-O-[2-(trimethylsilyl)ethoxymethyl]- $\alpha$-Dglucopyranosyluronate) sodium salt (10). A solution of $5(0.050 \mathrm{~g}, 0.048 \mathrm{mmol})$ in $90 \%$ aqueous $\mathrm{CH}_{3} \mathrm{CN}(3 \mathrm{~mL})$ was treated with $\mathrm{CAN}(0.079 \mathrm{~g}, 0.144 \mathrm{mmol})$ at $0{ }^{\circ} \mathrm{C}$, then stirred at 0 ${ }^{\circ} \mathrm{C}$ for $7 \mathrm{~h}$. The reaction was quenched with saturated aqueous $\mathrm{NaHSO}_{3}$ solution $(10 \mathrm{~mL})$, extracted with $\mathrm{CH}_{2} \mathrm{Cl}_{2}(3 \times 50 \mathrm{~mL})$, dried over $\mathrm{Na}_{2} \mathrm{SO}_{4}$, then concentrated under reduced pressure. Purification by silica gel chromatography (20\% EtOAc in hexanes] yielded the 4'-OH 
disaccharide as a clear viscous oil $(0.037 \mathrm{~g}, 84 \%)$. $[\alpha]_{\mathrm{D}}+96.6^{\circ}$ (c 1.8, $\left.\mathrm{CHCl}_{3}\right)$; IR (neat): 3509.0, 2109.5, 1750.2, $1721.4 \mathrm{~cm}^{-1} ;{ }^{1} \mathrm{H}$ NMR $\left(\mathrm{CDCl}_{3}, 300 \mathrm{MHz}\right): \delta 7.67-7.71(4 \mathrm{H}, \mathrm{m}), 7.37-7.46(6 \mathrm{H}$, m), $5.54(1 \mathrm{H}, \mathrm{d}, J=3.3 \mathrm{~Hz}), 5.32(1 \mathrm{H}, \mathrm{d}, J=9.6 \mathrm{~Hz}), 4.79-4.90(4 \mathrm{H}, \mathrm{m}), 4.22-4.26(1 \mathrm{H}, \mathrm{m})$, 3.95-4.08 (3H, m), 3.65-3.88 (3H, m), $3.63(3 \mathrm{H}, \mathrm{s}), 3.50-3.56(2 \mathrm{H}, \mathrm{m}), 3.39(3 \mathrm{H}, \mathrm{s}), 3.20(1 \mathrm{H}$, $\mathrm{dd}, J=3.3,10.5 \mathrm{~Hz}), 3.10-3.12(1 \mathrm{H}, \mathrm{m}), 2.82-2.87$ (2H, m), 2.62-2.69 (2H, m), 2.19 (3H, s), $2.13(3 \mathrm{H}, \mathrm{s}), 1.06(9 \mathrm{H}, \mathrm{s}), 0.91(2 \mathrm{H}, \mathrm{t}, J=8.4 \mathrm{~Hz}), 0.00(9 \mathrm{H}, \mathrm{s}) ;{ }^{13} \mathrm{C} \mathrm{NMR}\left(\mathrm{CDCl}_{3}, 125 \mathrm{MHz}\right)$ : $\delta 207.75,173.16,170.72,169.83,136.13,136.08,133.46,133.42,130.28,128.25,128.21,98.42$, 97.65, 97.34, 79.04, 75.62, 73.52, 72.42, 72.08, 70.38, 70.33, 67.00, 63.60, 61.41, 56.20, 53.11, 38.80, 30.24, 28.65, 27.32, 21.62, 19.77, 18.48, -0.97; ESIMS: $m / z$ 940.15, [M+ Na $]^{+}$.

A solution of 4'-OH disaccharide $(0.034 \mathrm{~g}, 0.037 \mathrm{mmol})$ in anhydrous pyridine $(1 \mathrm{~mL})$ was treated with $\mathrm{SO}_{3} \cdot \mathrm{NMe}_{3}$ complex $(0.112 \mathrm{~g}, 0.806 \mathrm{mmol})$. The reaction mixture was stirred at 65 ${ }^{\circ} \mathrm{C}$ in a pressure tube for $20 \mathrm{~h}$, then cooled to $0{ }^{\circ} \mathrm{C}$ and quenched with anhydrous methanol (5 $\mathrm{mL}$ ). The mixture was stirred at $\mathrm{rt}$ for $1 \mathrm{~h}$, and treated with a 1:1 mixture of saturated aqueous $\mathrm{NaHCO}_{3}$ solution and brine $(20 \mathrm{~mL})$, stirred for $1 \mathrm{~h}$, extracted with $\mathrm{CH}_{2} \mathrm{Cl}_{2}(4 \times 100 \mathrm{~mL})$, dried over $\mathrm{Na}_{2} \mathrm{SO}_{4}$, then concentrated under reduced pressure. Purification by silica gel chromatography $\left(5 \% \mathrm{MeOH}\right.$ in $\left.\mathrm{CH}_{2} \mathrm{Cl}_{2}\right)$ yielded disaccharide 4'-O-sulfate $\mathbf{1 0}$ as an amorphous white solid $(0.030 \mathrm{~g}, 85 \%)$. [ $\alpha]_{\mathrm{D}}+103.1^{\mathrm{o}}\left(\mathrm{c} 1.6, \mathrm{CHCl}_{3}\right)$; IR (neat): 2110.9, 1747.5, 1721.5, $1249.9 \mathrm{~cm}^{-1}$; ${ }^{1} \mathrm{H}$ NMR $\left(25 \% \mathrm{CD}_{3} \mathrm{OD} / \mathrm{CDCl}_{3}, 300 \mathrm{MHz}\right): \delta 7.46-7.49(4 \mathrm{H}, \mathrm{m}), 7.04-7.14(6 \mathrm{H}$, m), $5.33(1 \mathrm{H}, \mathrm{d}, J=3.6 \mathrm{~Hz}), 5.20(1 \mathrm{H}, \mathrm{t}, J=9.3 \mathrm{~Hz}), 4.67(1 \mathrm{H}, \mathrm{d}, J=3.0 \mathrm{~Hz}), 4.62(1 \mathrm{H}, \mathrm{d}, J=$ $6.6 \mathrm{~Hz}), 4.52-4.57(2 \mathrm{H}, \mathrm{m}), 4.22-4.30(1 \mathrm{H}, \mathrm{m}), 4.11(1 \mathrm{H}, \mathrm{d}, J=8.1 \mathrm{~Hz}), 3.79-3.85(2 \mathrm{H}, \mathrm{m})$, 3.65-3.77 (2H, m), 3.26-3.45 (3H, m), $3.39(3 \mathrm{H}, \mathrm{s}), 3.16(3 \mathrm{H}, \mathrm{s}), 2.99$ (1H, dd, J = 3.0, 10.8 Hz), 2.50-2.62 (2H, m), 2.36-2.44 (2H, m), $1.98(3 \mathrm{H}, \mathrm{s}), 1.92(3 \mathrm{H}, \mathrm{s}), 0.82(9 \mathrm{H}, \mathrm{s}), 0.72(2 \mathrm{H}, \mathrm{t}, J$ $=8.7 \mathrm{~Hz}),-0.76(9 \mathrm{H}, \mathrm{s}) ;{ }^{13} \mathrm{C}$ NMR (CD $\left.\mathrm{OD}, 125 \mathrm{MHz}\right): \delta 209.06,172.90,171.08,170.01$, 136.03, 134.04, 133.98, 129.63, 127.67, 97.56, 96.95, 96.40, 77.81, 74.59, 73.93, 72.31, 71.58, $71.15,70.58,66.70,63.17,61.54,55.43,52.39,52.35,37.78,28.76,28.33,26.54,20.24,19.28$, 18.02, -2.25; ESIMS: $m / z$ 1042.18, $[\mathrm{M}+\mathrm{Na}]^{+}, 996.24,[\mathrm{M}-\mathrm{Na}]^{-}$.

Methyl (methyl-4-O-[2-azido-6-O-(tert-butyldiphenylsilyl)-2-deoxy-4-O-(p-methoxybenzyl)3-Osulfonato- $\alpha$-D-glucopyranosyl]-2-O-acetyl-3-O-[2-(trimethylsilyl)ethoxymethyl]- $\alpha$-Dglucopyranosyluronate) sodium salt (11). A solution of $5(0.045 \mathrm{~g}, 0.043 \mathrm{mmol})$ in pyridine (1 $\mathrm{mL}$ ) was treated with a $1 \mathrm{M}$ hydrazine hydrate solution in a 3:2 mixture of pyridine and acetic 
acid $(0.430 \mathrm{~mL}, 0.430 \mathrm{mmol})$, then stirred at $\mathrm{rt}$ for $6 \mathrm{~h}$. The reaction mixture was concentrated under reduced pressure, and then dissolved in $\mathrm{CH}_{2} \mathrm{Cl}_{2}(200 \mathrm{~mL})$. The mixture was washed with saturated aqueous $\mathrm{NaHCO}_{3}$ solution $(20 \mathrm{~mL})$, brine $(20 \mathrm{~mL})$, dried over $\mathrm{Na}_{2} \mathrm{SO}_{4}$, then concentrated under reduced pressure. Purification by silica gel chromatography $(20 \%$ EtOAc in hexanes) yielded 3'-OH disaccharide as a clear viscous oil $(0.036 \mathrm{~g}, 93 \%)$. $[\alpha]_{\mathrm{D}}+119.5^{\circ}$ (c 2.1, $\mathrm{CHCl}_{3}$ ); IR (neat): 3484.7, 2109.6, 1750.1, $1613.2 \mathrm{~cm}^{-1} ;{ }^{1} \mathrm{H} \mathrm{NMR}\left(\mathrm{CDCl}_{3}, 300 \mathrm{MHz}\right): \delta 7.70$ $7.76(4 \mathrm{H}, \mathrm{m}), 7.32-7.48(6 \mathrm{H}, \mathrm{m}), 7.20(2 \mathrm{H}, \mathrm{d}, J=8.4 \mathrm{~Hz}), 6.86(2 \mathrm{H}, \mathrm{d}, J=8.4 \mathrm{~Hz}), 5.46(1 \mathrm{H}, \mathrm{d}$, $J=3.3 \mathrm{~Hz}), 4.96(1 \mathrm{H}, \mathrm{d}, J=6.6 \mathrm{~Hz}), 4.84-4.90(3 \mathrm{H}, \mathrm{m}), 4.74(2 \mathrm{H}, \mathrm{s}), 4.15-4.20$ (1H, m), 3.99$4.07(4 \mathrm{H}, \mathrm{m}), 3.89(1 \mathrm{H}, \mathrm{d}, J=11.1 \mathrm{~Hz}), 3.80(3 \mathrm{H}, \mathrm{s}), 3.71-3.79(2 \mathrm{H}, \mathrm{m}), 3.59(1 \mathrm{H}, \mathrm{t}, J=8.4$ Hz), $3.50(3 \mathrm{H}, \mathrm{s}), 3.45-3.53(1 \mathrm{H}, \mathrm{m}), 3.39(3 \mathrm{H}, \mathrm{s}), 3.23(1 \mathrm{H}, \mathrm{dd}, J=3.6,10.5 \mathrm{~Hz}), 2.71(1 \mathrm{H}, \mathrm{d}, J$ $=2.4 \mathrm{~Hz}), 2.16(3 \mathrm{H}, \mathrm{s}), 1.12(9 \mathrm{H}, \mathrm{s}), 0.97(2 \mathrm{H}, \mathrm{t}, J=8.4 \mathrm{~Hz}), 0.05(9 \mathrm{H}, \mathrm{s}) ;{ }^{13} \mathrm{C} \mathrm{NMR}\left(\mathrm{CDCl}_{3}\right.$, $125 \mathrm{MHz}): \delta 170.36,169.17,159.43,135.99,135.70,133.69,133.12,130.59,129.79,129.37$, 127.81. 127.69, 114.07, 98.23, 97.29, 97.09, 78.60, 77.78, 75.79, 74.62, 72.11, 71.99, 71.58, 70.28, 66.59, 63.37, 62.09, 55.77, 55.30, 52.53, 27.02, 21.22, 19.48, 18.13, -1.33; ESIMS: $\mathrm{m} / \mathrm{z}$ 961.97, $[\mathrm{M}+\mathrm{Na}]^{+}$.

A solution of 3'-OH disaccharide $(0.036 \mathrm{~g}, 0.038 \mathrm{mmol})$ in anhydrous pyridine $(1 \mathrm{~mL})$ was treated with $\mathrm{SO}_{3} \cdot \mathrm{NMe}_{3}$ complex $(0.106 \mathrm{~g}, 0.764 \mathrm{mmol})$. The reaction mixture was stirred at 55 ${ }^{\circ} \mathrm{C}$ in a pressure tube for $20 \mathrm{~h}$, then cooled to $0{ }^{\circ} \mathrm{C}$ and quenched with anhydrous methanol $(5$ $\mathrm{mL}$ ). The mixture was stirred at $\mathrm{rt}$ for $1 \mathrm{~h}$, treated with a 1:1 mixture of saturated aqueous $\mathrm{NaHCO}_{3}$ solution and brine $(20 \mathrm{~mL})$ and stirred for $1 \mathrm{~h}$, then extracted with dichloromethane (4 x $100 \mathrm{~mL}$ ), dried over $\mathrm{Na}_{2} \mathrm{SO}_{4}$, and concentrated under reduced pressure. Purification by silica gel chromatography (5\% $\mathrm{MeOH}$ in $\left.\mathrm{CH}_{2} \mathrm{Cl}_{2}\right)$ yielded disaccharide 3'-O-sulfate 11 as an amorphous white solid $(0.039 \mathrm{~g}, 95 \%)$. $[\alpha]_{\mathrm{D}}+92.6^{\circ}$ (c 0.9, $\left.\mathrm{CHCl}_{3}\right)$; IR (neat): 2115.1, 1749.2, 1514.1, $1248.3 \mathrm{~cm}^{-1} ;{ }^{1} \mathrm{H}$ NMR $\left(25 \% \mathrm{CD}_{3} \mathrm{OD} / \mathrm{CDCl}_{3}, 300 \mathrm{MHz}\right): \delta 7.59-7.64(4 \mathrm{H}, \mathrm{m}), 7.27-7.38$ $(6 \mathrm{H}, \mathrm{m}), 7.15(2 \mathrm{H}, \mathrm{d}, J=8.4 \mathrm{~Hz}), 6.69(2 \mathrm{H}, \mathrm{d}, J=8.4 \mathrm{~Hz}), 5.44(1 \mathrm{H}, \mathrm{d}, J=3.3 \mathrm{~Hz}), 5.07(1 \mathrm{H}, \mathrm{d}$, $J=9.9 \mathrm{~Hz}), 4.87(1 \mathrm{H}, \mathrm{d}, J=6.3 \mathrm{~Hz}), 4.63-4.81(4 \mathrm{H}, \mathrm{m}), 4.45(1 \mathrm{H}, \mathrm{d}, J=10.2 \mathrm{~Hz}), 4.11(1 \mathrm{H}, \mathrm{d}$, $J=8.7 \mathrm{~Hz}), 3.95-4.00(2 \mathrm{H}, \mathrm{m}), 3.70-3.88(3 \mathrm{H}, \mathrm{m}), 3.70(3 \mathrm{H}, \mathrm{s}), 3.64(1 \mathrm{H}, \mathrm{t}, J=9.0 \mathrm{~Hz}), 3.48-$ $3.55(1 \mathrm{H}, \mathrm{m}), 3.44(3 \mathrm{H}, \mathrm{s}), 3.38-3.45(1 \mathrm{H}, \mathrm{m}), 3.31(3 \mathrm{H}, \mathrm{s}), 3.17(1 \mathrm{H}, \mathrm{dd}, J=3.3,10.2 \mathrm{~Hz}), 2.05$ $(3 \mathrm{H}, \mathrm{s}), 0.99(9 \mathrm{H}, \mathrm{s}), 0.84(2 \mathrm{H}, \mathrm{t}, J=8.4 \mathrm{~Hz}), 0.03(9 \mathrm{H}, \mathrm{s}) ;{ }^{13} \mathrm{C} \mathrm{NMR}\left(\mathrm{CDCl}_{3}, 125 \mathrm{MHz}\right)$ : $\delta 170.80,169.66,159.64,136.33,136.09,134.01,133.57,130.66,130.56,130.11,128.16$, $128.05,114.18,97.84,97.13,96.62,79.19,76.19,75.09,73.56,71.75,71.18,66.95,63.10$, 
62.55, 56.48, 55.55, 53.07, 46.15, 27.32, 21.55, 19.75, 18.42, -0.97; ESIMS: m/z 1064.11, [M+ $\mathrm{Na}]^{+}, 1018.30,[\mathrm{M}-\mathrm{Na}]^{-}$.

Methyl (methyl 4-O-[2-azido-6-O-(tert-butyldiphenylsilyl)-2-deoxy-3-O-levulinoyl4-O-(p-methoxybenzyl)- $\alpha$-D-glucopyranosyl]-2-O-acetyl-3-O-sulfonato- $\alpha$-D-

glucopyranosyluronate) sodium salt (12). A suspension of $\mathrm{MgBr}_{2} \cdot \mathrm{Et}_{2} \mathrm{O}(0.099 \mathrm{~g}, 0.385 \mathrm{mmol})$ in anhydrous ethyl ether $(1 \mathrm{~mL})$ was stirred at $\mathrm{rt}$ until the solution became clear, then treated with dry nitromethane $(0.042 \mathrm{~mL}, 0.770 \mathrm{mmol})$, followed by a solution of $5(0.040 \mathrm{~g}, 0.038 \mathrm{mmol})$ in anhydrous ethyl ether $(1 \mathrm{~mL})$. The reaction mixture was stirred at $\mathrm{rt}$ for $12 \mathrm{~h}$, diluted with ethyl ether $(20 \mathrm{~mL})$, quenched at $0{ }^{\circ} \mathrm{C}$ with saturated aqueous $\mathrm{NaHCO}_{3}$ solution $(20 \mathrm{~mL})$, extracted with $\mathrm{CH}_{2} \mathrm{Cl}_{2}(3 \times 50 \mathrm{~mL})$, dried over $\mathrm{Na}_{2} \mathrm{SO}_{4}$, then concentrated under reduced pressure.

Purification by silica gel chromatography (30\% EtOAc in hexanes) yielded 3-OH disaccharide as a clear viscous oil $(0.026 \mathrm{~g}, 78 \%)$.

A solution of $3-\mathrm{OH}$ disaccharide $(0.026 \mathrm{~g}, 0.029 \mathrm{mmol})$ in anhydrous pyridine $(1 \mathrm{~mL})$ was treated with $\mathrm{SO}_{3} \cdot \mathrm{NMe}_{3}$ complex $(0.080 \mathrm{~g}, 0.573 \mathrm{mmol})$. The reaction mixture was stirred at 55 ${ }^{\circ} \mathrm{C}$ in a pressure tube for $20 \mathrm{~h}$, then cooled to $0{ }^{\circ} \mathrm{C}$ and quenched with anhydrous methanol $(5$ $\mathrm{mL}$ ). The mixture was stirred at $\mathrm{rt}$ for $1 \mathrm{~h}$, and treated with a 1:1 mixture of saturated aqueous $\mathrm{NaHCO}_{3}$ solution and brine $(20 \mathrm{~mL})$, stirred for $1 \mathrm{~h}$, extracted with $\mathrm{CH}_{2} \mathrm{Cl}_{2}(4 \times 50 \mathrm{~mL})$, dried over $\mathrm{Na}_{2} \mathrm{SO}_{4}$, then concentrated under reduced pressure. Purification by silica gel chromatography $\left(5 \% \mathrm{MeOH}\right.$ in $\left.\mathrm{CH}_{2} \mathrm{Cl}_{2}\right)$ yielded disaccharide 3-O-sulfate 12 as an amorphous white solid (0.029 g, 95\%). [ $\alpha]_{\mathrm{D}}+100.5^{\circ}$ (c 1.2, $\left.\mathrm{CHCl}_{3}\right)$; IR (neat): 2112.8, 1747.8. 1613.5, $1588.1 \mathrm{~cm}^{-1} ;{ }^{1} \mathrm{H} \mathrm{NMR}\left(25 \% \mathrm{CD}_{3} \mathrm{OD} / \mathrm{CDCl}_{3}, 300 \mathrm{MHz}\right): \delta 7.52-7.57(4 \mathrm{H}, \mathrm{m}), 7.20-7.30(6 \mathrm{H}$, $\mathrm{m}), 6.93(2 \mathrm{H}, \mathrm{d}, J=8.4 \mathrm{~Hz}), 6.65(2 \mathrm{H}, \mathrm{d}, J=8.4 \mathrm{~Hz}), 5.50(1 \mathrm{H}, \mathrm{d}, J=3.6 \mathrm{~Hz}), 5.27(1 \mathrm{H}, \mathrm{dd}, J$ $=9.0,9.3 \mathrm{~Hz}), 4.84-4.91(2 \mathrm{H}, \mathrm{m}), 4.73(1 \mathrm{H}, \mathrm{t}, J=6.9 \mathrm{~Hz}), 4.35-4.47(3 \mathrm{H}, \mathrm{m}), 4.11(1 \mathrm{H}, \mathrm{t}, J=$ $6.6 \mathrm{~Hz}), 3.73(2 \mathrm{H}, \mathrm{s}), 3.60-3.67(1 \mathrm{H}, \mathrm{m}), 3.64(3 \mathrm{H}, \mathrm{s}), 3.52-3.58(1 \mathrm{H}, \mathrm{m}), 3.53(3 \mathrm{H}, \mathrm{s}), 3.30(3 \mathrm{H}$, s), $3.00(1 \mathrm{H}, \mathrm{dd}, J=3.6,10.8 \mathrm{~Hz}), 2.60-2.65(2 \mathrm{H}, \mathrm{m}), 2.34-2.50(2 \mathrm{H}, \mathrm{m}), 2.05(3 \mathrm{H}, \mathrm{s}), 2.03$ $(3 \mathrm{H}, \mathrm{s}), 0.93(9 \mathrm{H}, \mathrm{s}) ;{ }^{13} \mathrm{C} \mathrm{NMR}\left(\mathrm{CDCl}_{3}, 125 \mathrm{MHz}\right): \delta 207.59,172.90,172.33,170.29,159.62$, 136.33, 136.08, 133.87, 133.34, 130.60, 130.22, 130.20, 129.76, 128.22, 128.14, 114.17, 99.35, 96.85, 76.02, 75.97, 75.72, 74.72, 72.85, 72.57, 69.01, 62.74, 61.91, 56.64, 55.70, 53.22, 38.28, 30.20, 28.50, 27.35, 21.56, 19.71; ESIMS: m/z 1032.11, [M+Na $]^{+}, 986.27,[\mathrm{M}-\mathrm{Na}]^{-}$. 


\section{Methyl (methyl 4-O-[2-azido-6-O-(tert-butyldiphenylsilyl)-2-deoxy-3-O-levulinoyl-4-O-(p-} methoxybenzyl)- $\alpha$-D-glucopyranosyl]-3-O-[2-(trimethylsilyl)ethoxymethyl]-2-O-sulfonatoQ-D-glucopyranosyluronate) sodium salt (13). A solution of 5 (0.045 g, $0.043 \mathrm{mmol})$ in dry $\mathrm{CH}_{2} \mathrm{Cl}_{2}(2 \mathrm{~mL})$ was treated with $\mathrm{Mg}(\mathrm{OMe})_{2}(1.1 \mathrm{~mL}, 0.6 \mathrm{M}$ solution in $\mathrm{MeOH})$. The reaction mixture was stirred at $\mathrm{rt}$ for $12 \mathrm{~h}$, quenched with saturated aqueous $\mathrm{NH}_{4} \mathrm{Cl}$ solution $(20 \mathrm{~mL})$, extracted with $\mathrm{CH}_{2} \mathrm{Cl}_{2}(3 \times 50 \mathrm{~mL})$, dried over $\mathrm{Na}_{2} \mathrm{SO}_{4}$, then concentrated under reduced pressure. Purification by silica gel chromatography (25\% EtOAc in hexanes) yielded 2-OH disaccharide as a clear viscous oil $(0.038 \mathrm{~g}, 88 \%)$. $[\alpha]_{\mathrm{D}}+108.8^{\circ}\left(\mathrm{c} 0.4, \mathrm{CHCl}_{3}\right)$; IR (neat): 3324.4, 2108.1, 1751.8, $1720.2 \mathrm{~cm}^{-1} ;{ }^{1} \mathrm{H} \mathrm{NMR}\left(\mathrm{CDCl}_{3}, 300 \mathrm{MHz}\right): \delta 7.68(4 \mathrm{H}, \mathrm{t}, J=8.1 \mathrm{~Hz})$, 7.34-7.44 (6H, m), $7.12(2 \mathrm{H}, \mathrm{d}, J=8.7 \mathrm{~Hz}), 6.80(2 \mathrm{H}, \mathrm{d}, J=8.7 \mathrm{~Hz}), 5.39-5.46(2 \mathrm{H}, \mathrm{m}), 4.93$ $(1 \mathrm{H}, \mathrm{d}, J=7.2 \mathrm{~Hz}), 4.77-4.86(3 \mathrm{H}, \mathrm{m}), 4.58(2 \mathrm{H}, \mathrm{AB}), 4.16(1 \mathrm{H}, \mathrm{d}, J=9.6 \mathrm{~Hz}), 3.81-3.98(6 \mathrm{H}$, m), $3.77(3 \mathrm{H}, \mathrm{s}), 3.56-3.62(2 \mathrm{H}, \mathrm{m}), 3.35-3.50(1 \mathrm{H}, \mathrm{m}), 3.56(3 \mathrm{H}, \mathrm{s}), 3.46(3 \mathrm{H}, \mathrm{s}), 3.15(1 \mathrm{H}, \mathrm{dd}$, $J=3.6,10.8 \mathrm{~Hz}), 2.70-2.76(2 \mathrm{H}, \mathrm{m}), 2.53-2.59$ (2H, m), 2.17 (3H, s), 1.08 (9H, s), 0.94-0.99 $(2 \mathrm{H}, \mathrm{m}), 0.02(9 \mathrm{H}, \mathrm{s}) ;{ }^{13} \mathrm{C} \mathrm{NMR}\left(\mathrm{CDCl}_{3}, 125 \mathrm{MHz}\right): \delta 206.61,172.30,169.95,159.63,136.36$, 136.07, 134.04, 133.53, 130.80, 130.19, 130.14, 129.68, 128.21, 128.06, 114.17, 100.13, 98.96, $96.95,86.42,75.87,75.73,74.81,72.76,72.46,71.22 .70 .21,66.95,62.36,62.22,56.19,55.71$, 53.02, 38.28, 30.28, 28.44, 27.41, 19.89, 18.53, -1.02; ESIMS: $m / z$ 1018.17, $[\mathrm{M}+\mathrm{Na}]^{+}$.

A solution of $2-\mathrm{OH}$ disaccharide $(0.038 \mathrm{~g}, 0.038 \mathrm{mmol})$ in anhydrous pyridine $(1 \mathrm{~mL})$ was treated with $\mathrm{SO}_{3} \cdot \mathrm{NMe}_{3}$ complex $(0.106 \mathrm{~g}, 0.764 \mathrm{mmol})$. The reaction mixture was stirred at 55 ${ }^{\circ} \mathrm{C}$ in a pressure tube for $20 \mathrm{~h}$, then cooled to $0{ }^{\circ} \mathrm{C}$ and quenched with anhydrous methanol $(5$ $\mathrm{mL}$ ). The mixture was stirred at $\mathrm{rt}$ for $1 \mathrm{~h}$, treated with a 1:1 mixture of saturated aqueous $\mathrm{NaHCO}_{3}$ solution and brine $(20 \mathrm{~mL})$ and stirred for $1 \mathrm{~h}$, extracted with $\mathrm{CH}_{2} \mathrm{Cl}_{2}(4 \times 50 \mathrm{~mL})$, dried over $\mathrm{Na}_{2} \mathrm{SO}_{4}$, then concentrated under reduced pressure. Purification by silica gel chromatography $\left(5 \% \mathrm{MeOH}\right.$ in $\left.\mathrm{CH}_{2} \mathrm{Cl}_{2}\right)$ yielded disaccharide 2-O-sulfate 13 as an amorphous white solid $(0.040 \mathrm{~g}, 96 \%)$. [ $\alpha]_{\mathrm{D}}+79.3^{\circ}$ (c 1.8, $\left.\mathrm{CHCl}_{3}\right)$; IR (neat): 2109.6, 1751.9, 1720.7, 1613.1, $1249.6 \mathrm{~cm}^{-1} ;{ }^{1} \mathrm{H}$ NMR $\left(25 \% \mathrm{CD}_{3} \mathrm{OD} / \mathrm{CDCl}_{3}, 300 \mathrm{MHz}\right): \delta 7.61-7.67$ (4H, m), 7.31-7.39 $(6 \mathrm{H}, \mathrm{m}), 7.05(2 \mathrm{H}, \mathrm{d}, J=8.4 \mathrm{~Hz}), 6.76(2 \mathrm{H}, \mathrm{d}, J=8.4 \mathrm{~Hz}), 5.71(1 \mathrm{H}, \mathrm{d}, J=3.3 \mathrm{~Hz}), 5.37(1 \mathrm{H}, \mathrm{t}$, $J=10.2 \mathrm{~Hz}), 5.08(1 \mathrm{H}, \mathrm{d}, J=3.0 \mathrm{~Hz}), 4.95(1 \mathrm{H}, \mathrm{d}, J=6.0 \mathrm{~Hz}), 4.90(1 \mathrm{H}, \mathrm{d}, J=6.0 \mathrm{~Hz}), 4.57$ $(1 \mathrm{H}, \mathrm{d}, J=10.8 \mathrm{~Hz}), 4.50(1 \mathrm{H}, \mathrm{d}, J=10.8 \mathrm{~Hz}), 4.24-4.27(1 \mathrm{H}, \mathrm{m}), 4.16(1 \mathrm{H}, \mathrm{t}, J=9.0 \mathrm{~Hz})$, 3.75-4.02 (5H, m), $3.74(3 \mathrm{H}, \mathrm{s}), 3.68-3.73(2 \mathrm{H}, \mathrm{m}), 3.52(3 \mathrm{H}, \mathrm{s}), 3.36(3 \mathrm{H}, \mathrm{s}), 3.34-3.37(1 \mathrm{H}$, m), 3.11(1H, dd, $J=3.3,10.8 \mathrm{~Hz}), 2.69-2.73(2 \mathrm{H}, \mathrm{m}), 2.51-2.57(2 \mathrm{H}, \mathrm{m}), 2.15(3 \mathrm{H}, \mathrm{s}), 1.05$ 
(9H, s), 0.89-0.96 (2H, m), -0.03 (9H, s); $\left.{ }^{13} \mathrm{C} \mathrm{NMR} \mathrm{(} \mathrm{CDCl}_{3}, 125 \mathrm{MHz}\right): \delta 206.92,172.63$, 170.23 , 159.64, 136.33, 136.07, 134.04, 133.35, 130.67, 130.27, 130.20, 129.85, 128.22, 128.16, 114.16, 98.01, 97.86, 96.93, 75.69, 75.50, 74.79, 73.04, 72.71, 70.92, 67.24, 62.52, 62.26, 56.44, 55.69, 53.03, 46.24, 38.27, 30.26, 28.45, 27.44, 19.86, 18.33, -0.98; ESIMS: m/z 1120.14, [M + $\mathrm{Na}]^{+}, 1074.30,[\mathrm{M}-\mathrm{Na}]^{-}$.

Methyl (methyl 4-O-[2-azido-2-deoxy-3-O-levulinoyl-6-O-sulfonato- $\alpha$-D-glucopyranosyl]-2-$O$-acetyl-3-O-[2-(trimethylsilyl)ethoxymethyl]- $\alpha$-D-glucopyranosyluronate) sodium salt

(14). A solution of disaccharide monosulfate 9 (0.031 g, $0.034 \mathrm{mmol})$ in $90 \%$ aqueous $\mathrm{CH}_{3} \mathrm{CN}$ (4 $\mathrm{mL})$ was treated with $\mathrm{CAN}(0.057 \mathrm{~g}, 0.102 \mathrm{mmol})$ at $0{ }^{\circ} \mathrm{C}$ and stirred for $7 \mathrm{~h}$. The reaction mixture was quenched at $0{ }^{\circ} \mathrm{C}$ with saturated aqueous $\mathrm{NaHSO}_{3}$ solution $(5 \mathrm{~mL})$. A 1:1 mixture of saturated aqueous $\mathrm{NaHCO}_{3}$ solution and brine $(20 \mathrm{~mL})$ was added and stirred at $\mathrm{rt}$ for $1 \mathrm{~h}$. The product was extracted with $\mathrm{CH}_{2} \mathrm{Cl}_{2}(3 \times 100 \mathrm{~mL})$, dried over $\mathrm{Na}_{2} \mathrm{SO}_{4}$, then concentrated under reduced pressure. Purification by silica gel chromatography $\left(5 \% \mathrm{MeOH}\right.$ in $\left.\mathrm{CH}_{2} \mathrm{Cl}_{2}\right)$ yielded disaccharide 6'-O-sulfate 14 as an amorphous white solid $(0.020 \mathrm{~g}, 77 \%)$. $[\alpha]_{\mathrm{D}}+271.7^{\circ}(\mathrm{c} 0.3$, $\mathrm{CHCl}_{3}$ ); IR (neat): 3468.7, 2110.1, 1747.1, $1239.1 \mathrm{~cm}^{-1} ;{ }^{1} \mathrm{H} \mathrm{NMR}\left(25 \% \mathrm{CD}_{3} \mathrm{OD} / \mathrm{CDCl}_{3}, 300\right.$ $\mathrm{MHz}): \delta 5.30(1 \mathrm{H}, \mathrm{d}, J=3.6 \mathrm{~Hz}), 5.10(1 \mathrm{H}, \mathrm{d}, J=9.3 \mathrm{~Hz}), 4.62-4.75(4 \mathrm{H}, \mathrm{m}), 4.19-4.24(1 \mathrm{H}$, m), 3.90-4.15 (4H, m), 3.66 (3H, s), 3.48-3.61 (2H, m), 3.30-3.44 (2H, m), 3.26 (3H, s), 3.18$3.20(1 \mathrm{H}, \mathrm{m}), 3.11(1 \mathrm{H}, \mathrm{dd}, J=3.6,10.8 \mathrm{~Hz}), 2.64-2.92(2 \mathrm{H}, \mathrm{m}), 2.46-2.54(2 \mathrm{H}, \mathrm{m}), 2.11(3 \mathrm{H}$, s), $2.03(3 \mathrm{H}, \mathrm{s}), 0.80(2 \mathrm{H}, \mathrm{t}, J=8.7 \mathrm{~Hz}),-0.10(9 \mathrm{H}, \mathrm{s}) ;{ }^{13} \mathrm{C} \mathrm{NMR}\left(\mathrm{CD}_{3} \mathrm{OD}, 125 \mathrm{MHz}\right): \delta 207.97$, 172.62, 170.92, 169.92, 98.75, 97.45, 96.94, 77.65, 76.86, 72.54, 71.81, 70.75, 66.56, 66.52, 65.41, 61.91, 55.27, 54.71, 54.66, 52.52, 52.47, 37.46, 28.75, 28.03, 20.20, 17.93, -2.27; ESIMS: $m / z$ 804.91, [M+Na $]^{+}, 758.19,[\mathrm{M}-\mathrm{Na}]^{-}$.

Methyl (methyl 4-O-[2-azido-6-O-(tert-butyldiphenylsilyl)-2-deoxy-4-O-sulfonato- $\alpha$-Dglucopyranosyl]-2-O-acetyl-3-O-[2-(trimethylsilyl)ethoxymethyl]- $\alpha$-D-

glucopyranosyluronate) sodium salt (15). A solution of disaccharide monosulfate 10 (0.025 g, $0.024 \mathrm{mmol})$ in pyridine $(1 \mathrm{~mL})$ was treated with a $1 \mathrm{M}$ hydrazine hydrate solution in a 3:2 mixture of pyridine and acetic acid $(0.244 \mathrm{~mL}, 0.244 \mathrm{mmol})$ at $\mathrm{rt}$. The reaction mixture was stirred for $6 \mathrm{~h}$, then concentrated under reduced pressure and redissolved in $\mathrm{CH}_{2} \mathrm{Cl}_{2}(20 \mathrm{~mL})$. A 1:1 mixture of saturated aqueous $\mathrm{NaHCO}_{3}$ solution and brine $(20 \mathrm{~mL})$ was added and stirred at $\mathrm{rt}$ 
for $1 \mathrm{~h}$. The product was extracted with $\mathrm{CH}_{2} \mathrm{Cl}_{2}(3 \times 100 \mathrm{~mL})$, dried over $\mathrm{Na}_{2} \mathrm{SO}_{4}$, then concentrated under reduced pressure. Purification by silica gel chromatography $(5 \% \mathrm{MeOH}$ in $\mathrm{CH}_{2} \mathrm{Cl}_{2}$ ) yielded disaccharide 4'-O-sulfate 15 as an amorphous white solid $(0.019 \mathrm{~g}, 81 \%)$. $[\alpha]_{\mathrm{D}}$ $+107.7^{\circ}\left(\mathrm{c} 0.8, \mathrm{CHCl}_{3}\right)$; IR (neat): 3350.2, 2110.5, 1747.8, $1236.5 \mathrm{~cm}^{-1} ;{ }^{1} \mathrm{H}$ NMR $(25 \%$ $\left.\mathrm{CD}_{3} \mathrm{OD} / \mathrm{CDCl}_{3}, 300 \mathrm{MHz}\right): \delta 7.60-764(4 \mathrm{H}, \mathrm{m}), 7.20-7.30(6 \mathrm{H}, \mathrm{m}), 5.35(1 \mathrm{H}, \mathrm{d}, J=3.6 \mathrm{~Hz})$, 4.78-4.81 (2H, m), 4.68-4.71 (2H, m), 4.32 (1H, t, $J=9.6 \mathrm{~Hz}), 4.12-4.16$ (1H, m), 4.00 (1H, t, $J$ $=10.5 \mathrm{~Hz}), 3.92-3.96(2 \mathrm{H}, \mathrm{m}), 3.76-3.85(3 \mathrm{H}, \mathrm{m}), 3.53-3.60(1 \mathrm{H}, \mathrm{m}), 3.44(3 \mathrm{H}, \mathrm{s}), 3.29(3 \mathrm{H}, \mathrm{s})$, 3.23-3.25 (1H, m), 3.07 (1H, dd, $J=3.6,10.5 \mathrm{~Hz}), 2.02(3 \mathrm{H}, \mathrm{s}), 1.89(1 \mathrm{H}, \mathrm{s}), 0.93(9 \mathrm{H}, \mathrm{s}), 0.81$ $(2 \mathrm{H}, \mathrm{t}, J=8.7 \mathrm{~Hz}),-0.11(9 \mathrm{H}, \mathrm{s}) ;{ }^{13} \mathrm{C} \mathrm{NMR}\left(\mathrm{CD}_{3} \mathrm{OD}, 125 \mathrm{MHz}\right): \delta 171.08,170.01,136.03$, 134.04, 133.99, 129.63, 127.62, 97.56, 96.95, 96.40, 77.81, 74.59, 73.93, 72.31, 71.58, 71.15, $70.58,66.70,63.17,61.54,55.43,52.39,52.36,28.76,28.34,20.24,19.28,18.02,-2.25$; ESIMS: $m / z$ 944.92, $[\mathrm{M}+\mathrm{Na}]^{+}, 898.44,[\mathrm{M}-\mathrm{Na}]^{-}$.

\section{Methyl (methyl 4-O-[2-acetamido-6-O-(tert-butyldiphenylsilyl)-2-deoxy-4-O-(p- methoxybenzyl)-3-O-sulfonato- $\alpha$-D-glucopyranosyl]-2-O-acetyl-3-O-[2-} (trimethylsilyl)ethoxymethyl]- $\alpha$-D-glucopyranosyluronate) sodium salt (16). A solution of disaccharide monosulfate $11(0.028 \mathrm{~g}, 0.027 \mathrm{mmol})$ in anhydrous pyridine $(1 \mathrm{~mL})$ was treated with $\mathrm{AcSH}(0.077 \mathrm{~mL}, 1.076 \mathrm{mmol})$ at $\mathrm{rt}$, then stirred for $44 \mathrm{~h}$. The reaction mixture was concentrated under reduced pressure, redissolved in $\mathrm{CH}_{2} \mathrm{Cl}_{2}(20 \mathrm{~mL})$, treated with a 1:1 mixture of saturated aqueous $\mathrm{NaHCO}_{3}$ solution and brine $(20 \mathrm{~mL})$, stirred for $1 \mathrm{~h}$, extracted with $\mathrm{CH}_{2} \mathrm{Cl}_{2}$ ( 3 x $100 \mathrm{~mL}$ ), dried over $\mathrm{Na}_{2} \mathrm{SO}_{4}$, then concentrated under reduced pressure. Purification by silica gel chromatography $\left(5 \% \mathrm{MeOH}\right.$ in $\left.\mathrm{CH}_{2} \mathrm{Cl}_{2}\right)$ yielded disaccharide 3'-O-sulfate 16 as an amorphous white solid $(0.025 \mathrm{~g}, 89 \%)$. $[\alpha]_{\mathrm{D}}+66.4^{\circ}$ (c 1.5, $\left.\mathrm{CHCl}_{3}\right)$; IR (neat): 3472.9, 1748.6, $1248.2 \mathrm{~cm}^{-1}$; ${ }^{1} \mathrm{H}$ NMR $\left(25 \% \mathrm{CD}_{3} \mathrm{OD} / \mathrm{CDCl}_{3}, 300 \mathrm{MHz}\right): \delta 7.42-7.49(4 \mathrm{H}, \mathrm{m}), 7.10-7.20(6 \mathrm{H}$, m), $6.98(2 \mathrm{H}, \mathrm{d}, J=8.7 \mathrm{~Hz}), 6.53(2 \mathrm{H}, \mathrm{d}, J=8.7 \mathrm{~Hz}), 5.14(1 \mathrm{H}, \mathrm{d}, J=3.3 \mathrm{~Hz}), 4.89(1 \mathrm{H}, \mathrm{d}, J=$ $9.9 \mathrm{~Hz}), 4.43-4.63(5 \mathrm{H}, \mathrm{m}), 4.21-4.28(2 \mathrm{H}, \mathrm{m}), 3.90(1 \mathrm{H}, \mathrm{d}, J=8.7 \mathrm{~Hz}), 3.63-3.81(5 \mathrm{H}, \mathrm{m})$, 3.42-3.56 (2H, m), $3.54(3 \mathrm{H}, \mathrm{s}), 3.24-3.33(2 \mathrm{H}, \mathrm{m}), 3.24(3 \mathrm{H}, \mathrm{s}), 3.15(3 \mathrm{H}, \mathrm{s}), 1.91(3 \mathrm{H}, \mathrm{s}), 1.80$ $(3 \mathrm{H}, \mathrm{s}), 0.84(9 \mathrm{H}, \mathrm{s}), 0.69(2 \mathrm{H}, \mathrm{t}, J=8.7 \mathrm{~Hz}),-0.22(9 \mathrm{H}, \mathrm{s}) ;{ }^{13} \mathrm{C} \mathrm{NMR}\left(\mathrm{CD}_{3} \mathrm{OD}, 125 \mathrm{MHz}\right)$ : $\delta 172.90,171.01,169.93,159.66,136.13,135.86,133.86,133.46,131.10,130.13,129.90$, $129.87,127.88,127.76,113.52,98.09,97.43,96.77,78.31,76.12,75.82,74.62,73.05,71.89$, 
$70.83,66.44,62.59,55.13,54.66,54.37,52.29,26.51,22.18,20.27,19.20,17.92,-2.27$;

ESIMS: $m / z$ 1080.00, $[\mathrm{M}+\mathrm{Na}]^{+}, 1034.43,[\mathrm{M}-\mathrm{Na}]^{-}$.

Methyl (methyl 4-O-[2-azido-6-O-(tert-butyldiphenylsilyl)-2-deoxy-3-O-levulinoyl-4-O-(pmethoxybenzyl)- $\alpha$-D-glucopyranosyl]-3-O-sulfonato- $\alpha$-D-glucopyranosyluronate) sodium salt (17). A solution of disaccharide monosulfate $12(0.022 \mathrm{~g}, 0.022 \mathrm{mmol})$ in dry $\mathrm{CH}_{2} \mathrm{Cl}_{2}(2$ $\mathrm{mL})$ was treated with $\mathrm{Mg}(\mathrm{OMe})_{2}(0.54 \mathrm{~mL}, 0.6 \mathrm{M}$ solution in $\mathrm{MeOH})$ and stirred at $\mathrm{rt}$ for $12 \mathrm{~h}$, then quenched with saturated aqueous $\mathrm{NH}_{4} \mathrm{Cl}$ solution $(2 \mathrm{~mL})$. A 1:1 mixture of saturated aqueous $\mathrm{NaHCO}_{3}$ solution and brine $(20 \mathrm{~mL})$ was added, stirred for $1 \mathrm{~h}$, extracted with dichloromethane $(3 \times 100 \mathrm{~mL})$, dried over $\mathrm{Na}_{2} \mathrm{SO}_{4}$, then concentrated under reduced pressure. Purification by silica gel chromatography $\left(5 \% \mathrm{MeOH}\right.$ in $\mathrm{CH}_{2} \mathrm{Cl}_{2}$ ] yielded disaccharide 3-Osulfate 17 as an amorphous white solid $(0.018 \mathrm{~g}, 86 \%)$. $[\alpha]_{\mathrm{D}}+110.8^{\circ}$ (c $\left.0.9, \mathrm{CHCl}_{3}\right)$; IR (neat): 3424.0, 2114.0, 1750.7, 1719.9, $1249.5 \mathrm{~cm}^{-1}$; ${ }^{1} \mathrm{H}$ NMR $\left(25 \% \mathrm{CD}_{3} \mathrm{OD} / \mathrm{CDCl}_{3}, 300 \mathrm{MHz}\right)$ : $\delta 7.53-7.58(4 \mathrm{H}, \mathrm{m}), 7.23-7.30(6 \mathrm{H}, \mathrm{m}), 6.95(2 \mathrm{H}, \mathrm{d}, J=8.7 \mathrm{~Hz}), 6.66(2 \mathrm{H}, \mathrm{d}, J=8.7 \mathrm{~Hz}), 5.73$ $(1 \mathrm{H}, \mathrm{d}, J=3.6 \mathrm{~Hz}), 5.23(1 \mathrm{H}, \mathrm{t}, J=9.3 \mathrm{~Hz}), 4.66(1 \mathrm{H}, \mathrm{d}, J=3.3 \mathrm{~Hz}), 4.62(1 \mathrm{H}, \mathrm{t}, J=9.3 \mathrm{~Hz})$, $4.66(1 \mathrm{H}, \mathrm{d}, J=11.1 \mathrm{~Hz}), 4.60(1 \mathrm{H}, \mathrm{d}, J=11.2 \mathrm{~Hz}), 4.09(1 \mathrm{H}, \mathrm{d}, J=10.2 \mathrm{~Hz}), 3.85-3.93(2 \mathrm{H}$, m), 3.67-3.75 (4H, m), $3.65(3 \mathrm{H}, \mathrm{s}), 3.50(3 \mathrm{H}, \mathrm{s}), 3.29(3 \mathrm{H}, \mathrm{s}), 3.20-3.23(1 \mathrm{H}, \mathrm{m}), 3.02(1 \mathrm{H}, \mathrm{dd}$, $J=3.6,11.1 \mathrm{~Hz}), 2.63(2 \mathrm{H}, \mathrm{t}, J=6.6 \mathrm{~Hz}), 2.36-2.46(2 \mathrm{H}, \mathrm{m}), 2.06(3 \mathrm{H}, \mathrm{s}), 0.95(9 \mathrm{H}, \mathrm{s}) ;{ }^{13} \mathrm{C}$ NMR (CD 3 OD, $125 \mathrm{MHz}): \delta 209.68,174.33,172.25,161.43,137.73,137.43,135.44,134.74$, 132.18, 131.49, 131.25, 129.52, 118.98, 101.54, 98.16, 98.14, 82.84, 77.46, 76.07, 74.17, 74.09, $73.14,73.03$, 71.85, 64.11, 63.24, 56.74, 56.29, 54.07, 39.10, 30.33, 30.30, 29.67, 28.10, 20.78; ESIMS: $m / z$ 990.93, $[\mathrm{M}+\mathrm{Na}]^{+}, 944.33,[\mathrm{M}-\mathrm{Na}]^{-}$.

Methyl (methyl 4-O-[6-O-(tert-butyldiphenylsilyl)-2-azido-4-O-(p-methoxybenzyl)-2-deoxy3-O-levulinoyl- $\alpha$-D-glucopyranosyl]-2-O-sulfonato- $\alpha$-D-glucopyranosyluronate) sodium salt (18). A suspension of $\mathrm{MgBr}_{2} \cdot \mathrm{Et}_{2} \mathrm{O}(0.071 \mathrm{~g}, 0.273 \mathrm{mmol})$ in anhydrous ethyl ether $(1 \mathrm{~mL})$ was stirred at rt until the solution became clear, then treated with nitromethane $(0.030 \mathrm{~mL}, 0.546$ $\mathrm{mmol})$, followed by a solution of $13(0.030 \mathrm{~g}, 0.027 \mathrm{mmol})$ in anhydrous ethyl ether $(1 \mathrm{~mL})$. The reaction mixture was stirred at $\mathrm{rt}$ for $12 \mathrm{~h}$, diluted with ethyl ether $(20 \mathrm{~mL})$, quenched at $0{ }^{\circ} \mathrm{C}$ with a 1:1 mixture of saturated aqueous $\mathrm{NaHCO}_{3}$ solution and brine $(20 \mathrm{~mL})$, stirred for $1 \mathrm{~h}$, extracted with dichloromethane $(3 \times 100 \mathrm{~mL})$, dried over $\mathrm{Na}_{2} \mathrm{SO}_{4}$, then concentrated under 
reduced pressure. Purification by silica gel chromatography $\left(5 \% \mathrm{MeOH}\right.$ in $\left.\mathrm{CH}_{2} \mathrm{Cl}_{2}\right)$ yielded disaccharide 2-O-sulfate 18 as an amorphous white solid $(0.021 \mathrm{~g}, 80 \%)$. $[\alpha]_{\mathrm{D}}+48.5^{\circ}$ (c 1.0, $\left.\mathrm{CHCl}_{3}\right)$; IR (neat): $3451.3,2113.7,1749.9,1720.4,1249.1 \mathrm{~cm}^{-1} ;{ }^{1} \mathrm{H} \mathrm{NMR}\left(25 \% \mathrm{CD}_{3} \mathrm{OD} / \mathrm{CDCl}_{3}\right.$, $300 \mathrm{MHz}): \delta 7.50-7.56(4 \mathrm{H}, \mathrm{m}), 7.21-7.29(6 \mathrm{H}, \mathrm{m}), 6.95(2 \mathrm{H}, \mathrm{d}, J=8.4 \mathrm{~Hz}), 6.67(2 \mathrm{H}, \mathrm{d}, J=$ $8.4 \mathrm{~Hz}), 5.52(1 \mathrm{H}, \mathrm{d}, J=3.3 \mathrm{~Hz}), 5.28(1 \mathrm{H}, \mathrm{t}, J=9.6 \mathrm{~Hz}), 4.90(1 \mathrm{H}, \mathrm{d}, J=3.6 \mathrm{~Hz}), 4.47(1 \mathrm{H}, \mathrm{d}$, $J=11.1 \mathrm{~Hz}), 4.42(1 \mathrm{H}, \mathrm{d}, J=11.1 \mathrm{~Hz}), 4.10(1 \mathrm{H}, \mathrm{dd}, J=3.6,9.9 \mathrm{~Hz}), 3.92-4.07(3 \mathrm{H}, \mathrm{m})$, 3.70-3.85 (4H, m), $3.65(3 \mathrm{H}, \mathrm{s}), 3.34(3 \mathrm{H}, \mathrm{s}), 3.25(3 \mathrm{H}, \mathrm{s}), 3.20-3.22(1 \mathrm{H}, \mathrm{m}), 3.08(1 \mathrm{H}, \mathrm{dd}, J=$ 3.6, $10.8 \mathrm{~Hz}), 2.60-2.63(2 \mathrm{H}, \mathrm{m}), 2.37-2.50(2 \mathrm{H}, \mathrm{m}), 2.03(3 \mathrm{H}, \mathrm{s}), 0.94(9 \mathrm{H}, \mathrm{s}) ;{ }^{13} \mathrm{C}$ NMR $\left(\mathrm{CD}_{3} \mathrm{OD}, 125 \mathrm{MHz}\right): \delta 208.10,172.74,170.42,159.85,136.11,135.85,133.81,133.26,130.61$, $129.97,129.61,127.92,127.81,113.76,98.87,98.26,77.33,76.06,75.84,74.50,72.56,72.23$, 69.91, 62.30, 61.76, 55.34, 54.73, 54.70, 53.84, 52.21, 52.19, 37.50, 28.74, 28.07, 26.54, 26.51, 19.24; ESIMS: $m / z$ 990.8, [M+ Na $]^{+}, 944.30,[\mathrm{M}-\mathrm{Na}]^{-}$.

\section{Methyl (methyl 4-O-[6-O-(tert-butyldiphenylsilyl)-2-deoxy-4-O-(p-methoxybenzyl)-2-} sulfoamino- $\alpha$-D-glucopyranosyl]-2- $O$-acetyl-3- $O$-[2-(trimethylsilyl)ethoxymethyl]- $\alpha$-Dglucopyranosyluronate) sodium salt (19). A solution of 3'-OH disaccharide ( 0.070 g, 0.074 mmol) in dry $\mathrm{CH}_{2} \mathrm{Cl}_{2}(2 \mathrm{~mL})$ was treated with tributylphosphine $(0.028 \mathrm{~mL}, 0.112 \mathrm{mmol})$ under an argon atmosphere. The reaction mixture was stirred at $\mathrm{rt}$ for $5 \mathrm{~h}$, then quenched with water $(0.5 \mathrm{~mL})$ and stirred at $\mathrm{rt}$ for $14 \mathrm{~h}$. The reaction mixture was then treated with methanol $(3 \mathrm{~mL})$ and concentrated under reduced pressure. Purification by silica gel chromatography ( $3 \% \mathrm{MeOH}$ in $\mathrm{CH}_{2} \mathrm{Cl}_{2}$ ) yielded the disaccharide amine as a clear viscous oil $(0.048 \mathrm{~g}, 71 \%)$. $[\alpha]_{\mathrm{D}}+92.3^{\circ}$ (c 2.0, $\mathrm{CHCl}_{3}$ ); IR (neat): $3380.5,1749.8 \mathrm{~cm}^{-1} ;{ }^{1} \mathrm{H} \mathrm{NMR}\left(\mathrm{CDCl}_{3}, 300 \mathrm{MHz}\right): \delta 7.66-7.74(4 \mathrm{H}, \mathrm{m})$, 7.32-7.44 (6H, m), $7.20(2 \mathrm{H}, \mathrm{d}, J=8.4 \mathrm{~Hz}), 6.83(2 \mathrm{H}, \mathrm{d}, J=8.4 \mathrm{~Hz}), 5.17(1 \mathrm{H}, \mathrm{d}, J=3.3 \mathrm{~Hz})$, $4.88(1 \mathrm{H}, \mathrm{d}, J=3.6 \mathrm{~Hz}), 4.78-4.86(4 \mathrm{H}, \mathrm{m}), 4.66(1 \mathrm{H}, \mathrm{d}, J=10.8 \mathrm{~Hz}), 3.94-4.11(4 \mathrm{H}, \mathrm{m})$, 3.72-3.82 (2H, m), $3.80(3 \mathrm{H}, \mathrm{s}), 3.57-3.65(3 \mathrm{H}, \mathrm{m}), 3.45(1 \mathrm{H}, \mathrm{t}, J=10.2 \mathrm{~Hz}), 3.43(3 \mathrm{H}, \mathrm{s}), 3.37$ $(3 \mathrm{H}, \mathrm{s}), 2.67(1 \mathrm{H}, \mathrm{dd}, J=3.6,10.2 \mathrm{~Hz}), 2.09-2.17(2 \mathrm{H}, \mathrm{m}), 2.15(3 \mathrm{H}, \mathrm{s}), 1.07(9 \mathrm{H}, \mathrm{s}), 0.93(2 \mathrm{H}$, dd, $J=7.2,9.9 \mathrm{~Hz}), 0.02(9 \mathrm{H}, \mathrm{s}) ;{ }^{13} \mathrm{C} \mathrm{NMR}\left(\mathrm{CDCl}_{3}, 125 \mathrm{MHz}\right): \delta 171.52,170.30,160.57$, 137.37, 137.09, 135.24, 134.74, 132.50, 131.04, 130.72, 129.09, 128.99, 115.24, 102.27, 98.55, $98.35,79.58,79.06,77.45,75.51,75.20,73.88,73.77,72.20,68.03,63.80,57.92,57.12,56.69$, 53.90, 28.35, 22.54, 20.85, 19.57, 0.04; ESIMS: $\mathrm{m} / \mathrm{z}$ 914.93, [M+ H] ${ }^{+}, 936.20,[\mathrm{M}+\mathrm{Na}]^{+}$. 
A solution of the disaccharide amine $(0.035 \mathrm{~g}, 0.038 \mathrm{mmol})$ in dry $\mathrm{CH}_{2} \mathrm{Cl}_{2}(2 \mathrm{~mL})$ was treated with phenyl chlorosulfate $(0.021 \mathrm{~mL}, 0.152 \mathrm{mmol})$ and triethylamine $(0.021 \mathrm{~mL}, 0.152$ $\mathrm{mmol}$ ) at $0{ }^{\circ} \mathrm{C}$. After $10 \mathrm{~min}$ the ice bath was removed and stirring continued at $\mathrm{rt}$ for $14 \mathrm{~h}$. The reaction mixture was again cooled to $0{ }^{\circ} \mathrm{C}$ and treated with saturated aqueous $\mathrm{NaHCO}_{3}$ solution $(10 \mathrm{~mL})$. The ice bath was removed after $10 \mathrm{~min}$ and the mixture was stirred vigorously for an additional $1 \mathrm{~h}$ at $\mathrm{rt}$. The mixture was extracted with $\mathrm{CH}_{2} \mathrm{Cl}_{2}(3 \times 100 \mathrm{~mL})$, dried over $\mathrm{Na}_{2} \mathrm{SO}_{4}$, then concentrated under reduced pressure. Purification by silica gel chromatography $(10 \%$ $\mathrm{MeOH}$ in $\left.\mathrm{CH}_{2} \mathrm{Cl}_{2}\right)$ yielded disaccharide $N$-sulfate 19 as an amorphous white solid $(0.029 \mathrm{~g}$, $75 \%$ ). $[\alpha]_{\mathrm{D}}+76.2^{\circ}$ (c 1.5, $\mathrm{CHCl}_{3}$ ); IR (neat): $3443.2,1749.1,1248.3,1046.8 \mathrm{~cm}^{-1} ;{ }^{1} \mathrm{H} \mathrm{NMR}$ $\left(25 \% \mathrm{CD}_{3} \mathrm{OD} / \mathrm{CDCl}_{3}, 300 \mathrm{MHz}\right): \delta 7.54-7.61(4 \mathrm{H}, \mathrm{m}), 7.19-7.33(6 \mathrm{H}, \mathrm{m}), 7.06(2 \mathrm{H}, \mathrm{d}, J=8.4$ $\mathrm{Hz}), 6.70(2 \mathrm{H}, \mathrm{d}, J=8.4 \mathrm{~Hz}), 5.26\left(1 \mathrm{H}, \mathrm{d}, J=3.0 \mathrm{H}_{\mathrm{Z}}\right), 4.69-4.84(5 \mathrm{H}, \mathrm{m}), 4.51(1 \mathrm{H}, \mathrm{d}, J=10.2$ Hz), 3.88-3.99 (4H, m), 3.69 (3H, s), 3.55-3.67 (4H, m), 3.42-3.45 (2H, m), 3.29 (3H, s), 3.25 $(3 \mathrm{H}, \mathrm{s}), 3.16-3.18(1 \mathrm{H}, \mathrm{m}), 2.02(3 \mathrm{H}, \mathrm{s}), 0.95(9 \mathrm{H}, \mathrm{s}), 0.82(2 \mathrm{H}, \mathrm{t}, J=8.7 \mathrm{~Hz}),-0.11(9 \mathrm{H}, \mathrm{s}) ;{ }^{13} \mathrm{C}$ NMR (CD $\left.{ }_{3} \mathrm{OD}, 125 \mathrm{MHz}\right): \delta 170.97,169.65,159.66,136.11,135.87,133.81,133.53,131.25$, 129.90, 129.87, 129.41, 127.87, 127.79, 113.77, 113.68, 99.64, 97.43, 96.63, 78.04, 77.49, 76.83, 74.52, 73.32, 72.53, 72.17, 71.44, 66.25, 62.81, 59.07, 55.17, 54.72, 52.11, 26.52, 20.24, 19.21, 18.00,-2.23; ESIMS: $m / z$ 1038.00, $[\mathrm{M}+\mathrm{Na}]^{+}, 992.35,[\mathrm{M}-\mathrm{Na}]^{-}$. 
Fan, R. H.; Achkar, J.; Hernández-Torres, J. M.; Wei, A.
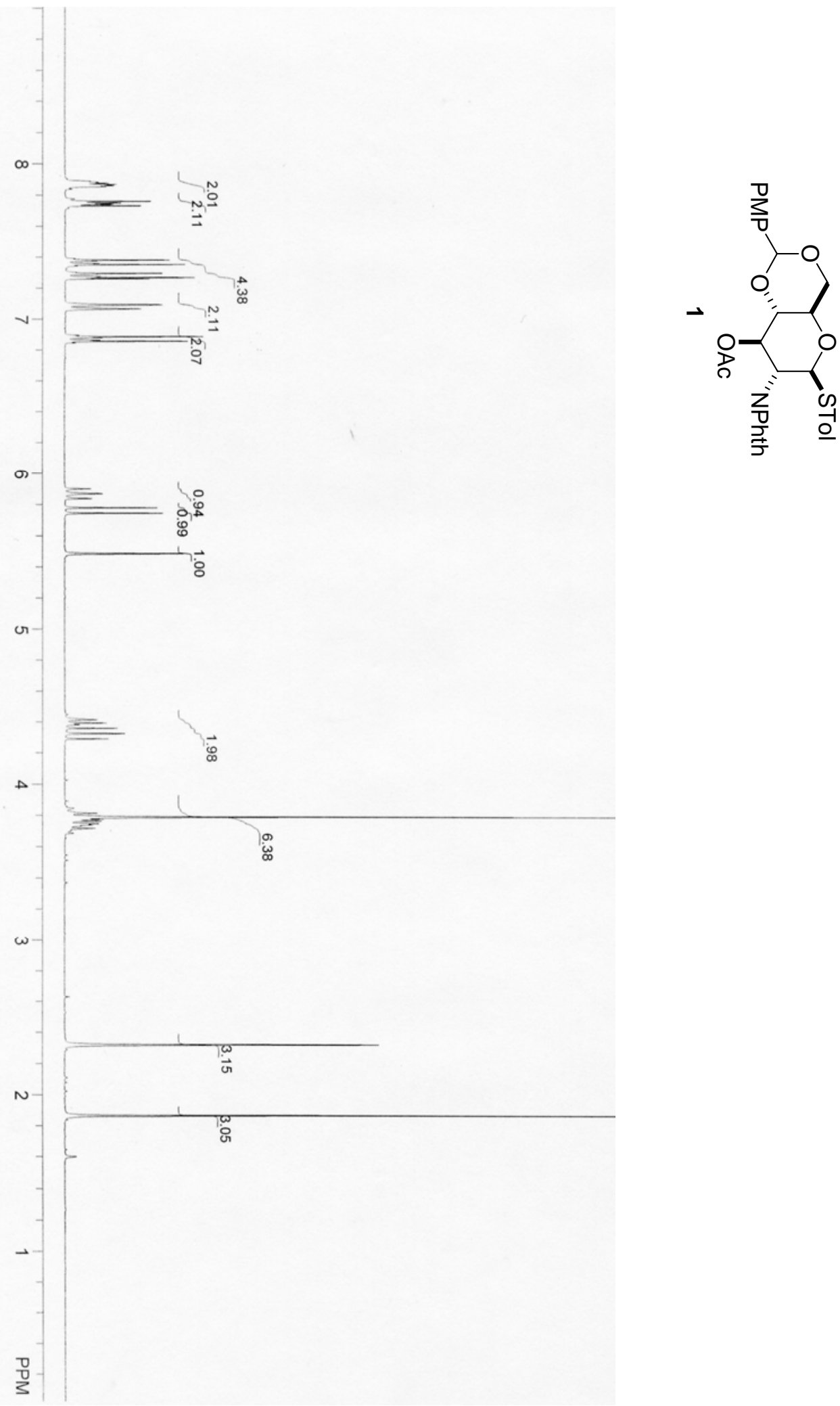

${ }^{1} \mathrm{H}$ NMR (300 MHz, $\mathrm{CDCl}_{3}$ ) Glucosamine Derivative 1 
Fan, R. H.; Achkar, J.; Hernández-Torres, J. M.; Wei, A.

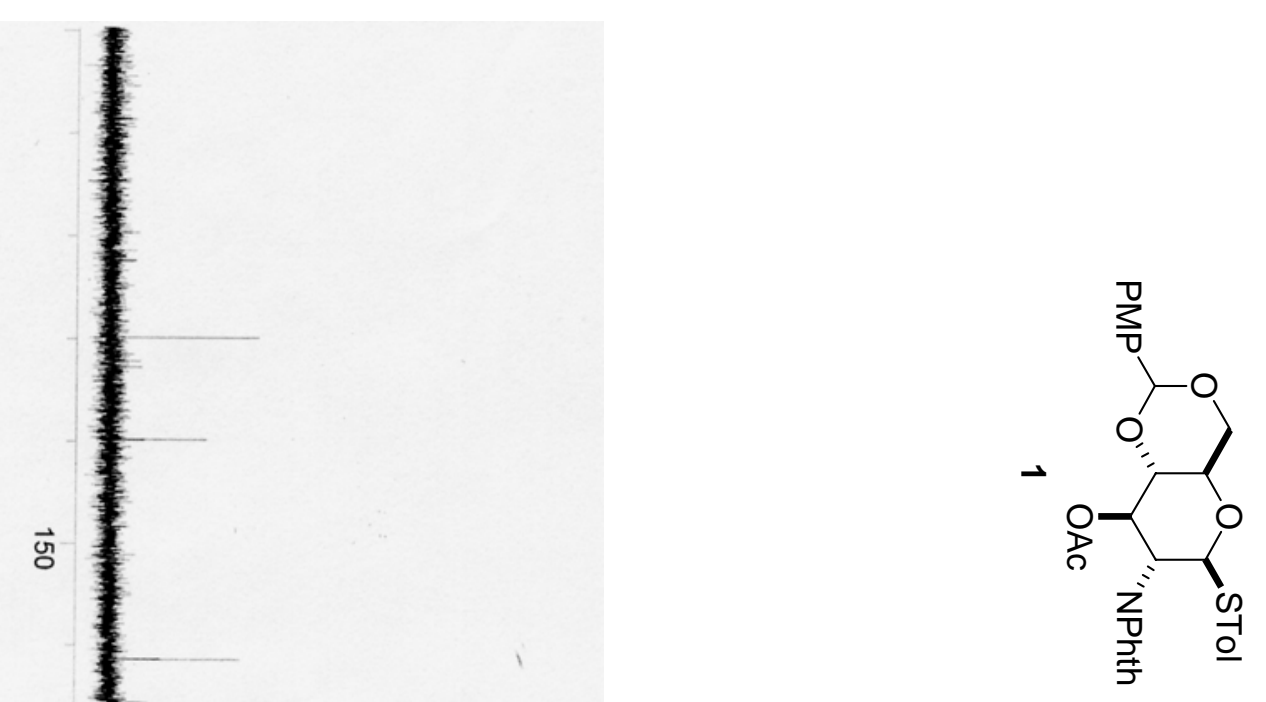

잉

$\vec{\circ}$

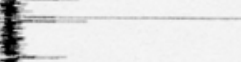

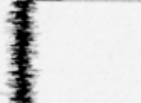

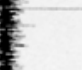

$-1$
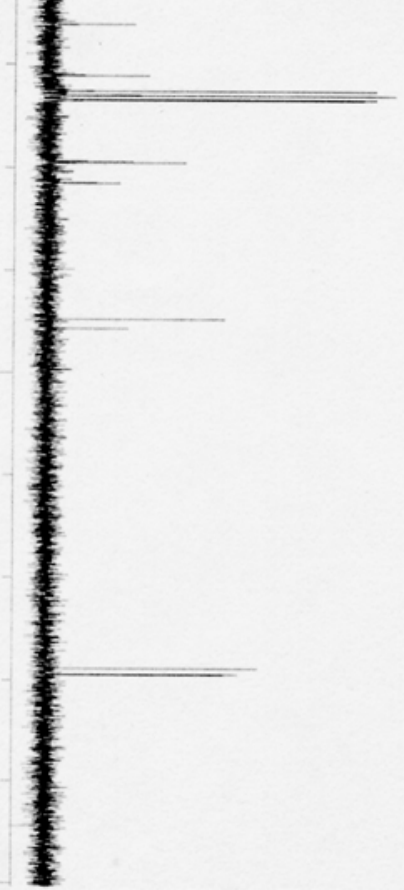

高

${ }^{13} \mathrm{C}$ NMR (300 MHz, $\mathrm{CDCl}_{3}$ ) Glucosamine Derivative 1 
Fan, R. H.; Achkar, J.; Hernández-Torres, J. M.; Wei, A.
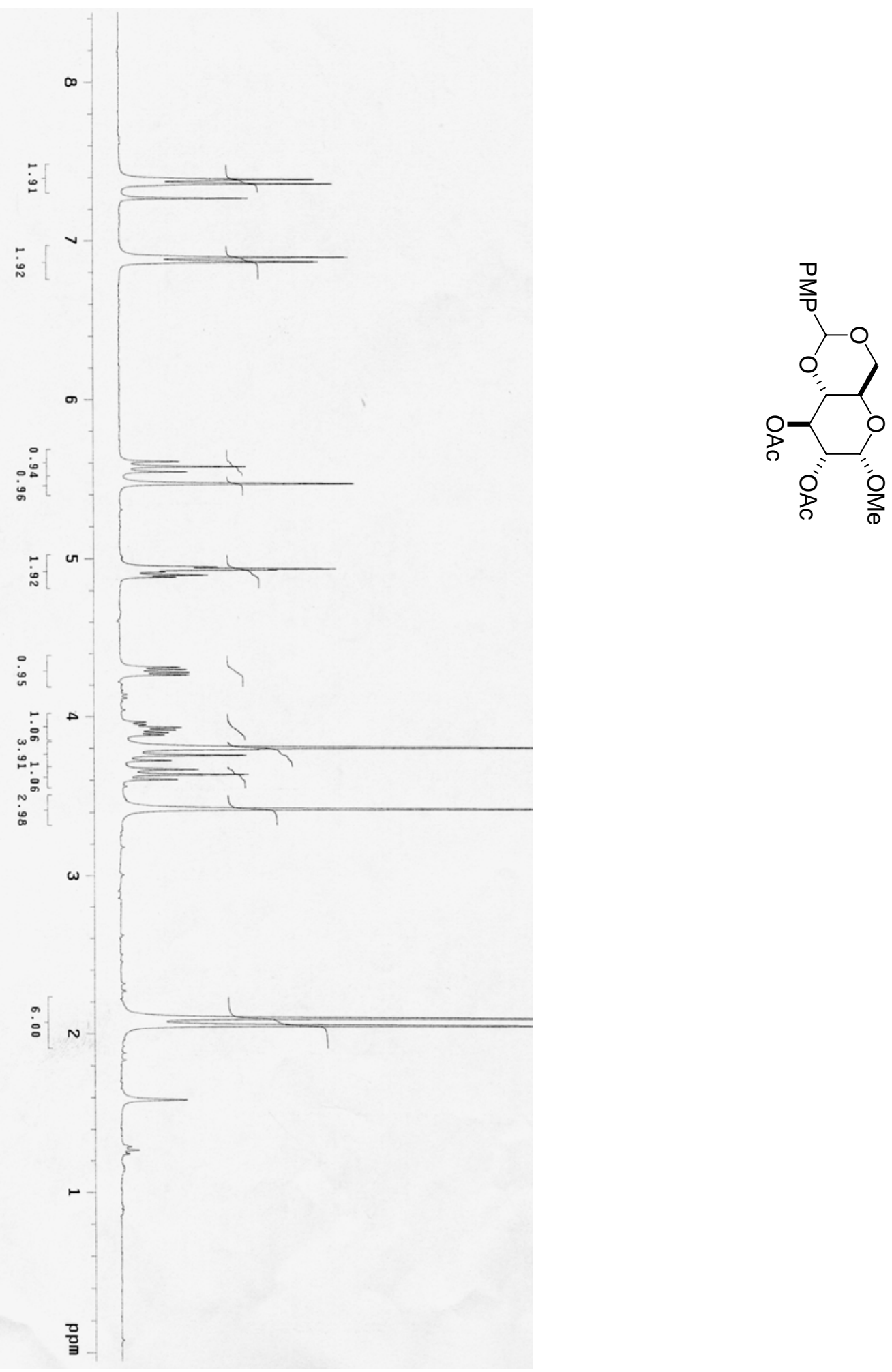

${ }^{1} \mathrm{H}$ NMR (300 MHz, $\mathrm{CDCl}_{3}$ ) Methyl Glucoside 2 
Fan, R. H.; Achkar, J.; Hernández-Torres, J. M.; Wei, A.
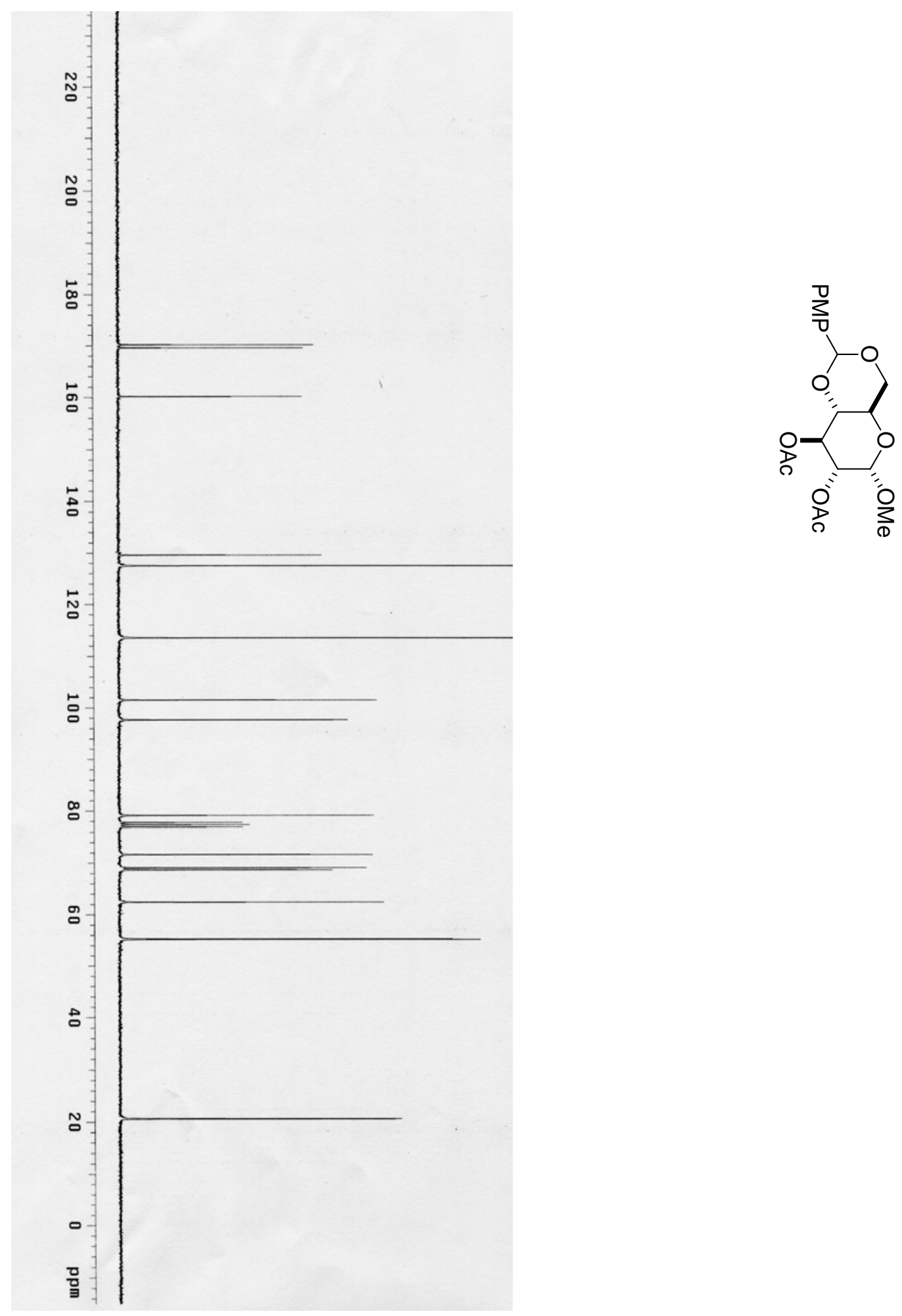

${ }^{13} \mathrm{C}$ NMR (300 MHz, $\mathrm{CDCl}_{3}$ ) Methyl Glucoside 2 
Fan, R. H.; Achkar, J.; Hernández-Torres, J. M.; Wei, A.
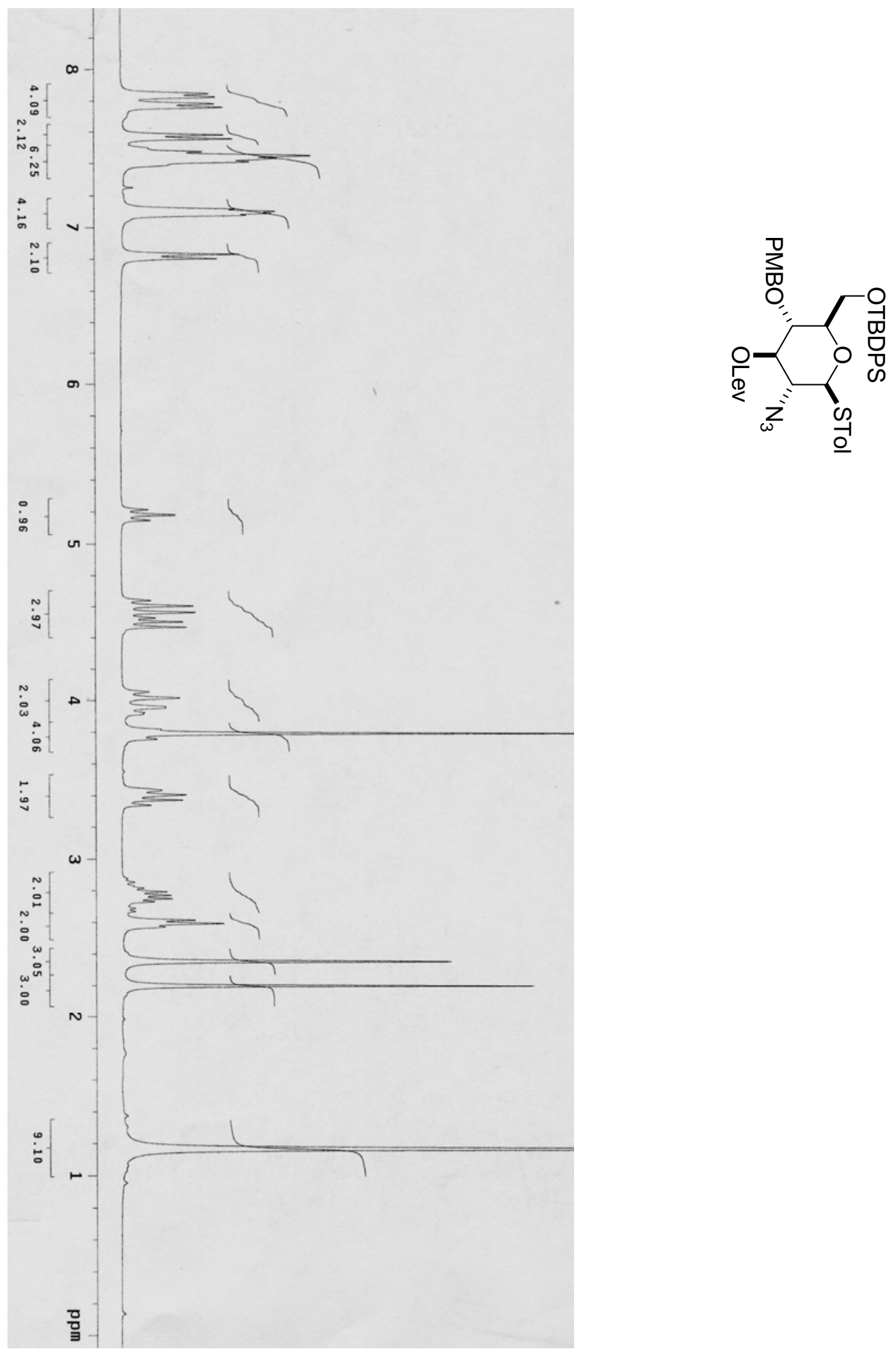

${ }^{1} \mathrm{H}$ NMR (300 MHz, $\left.\mathrm{CDCl}_{3}\right)$ Thioglycoside Donor 3 
Fan, R. H.; Achkar, J.; Hernández-Torres, J. M.; Wei, A.
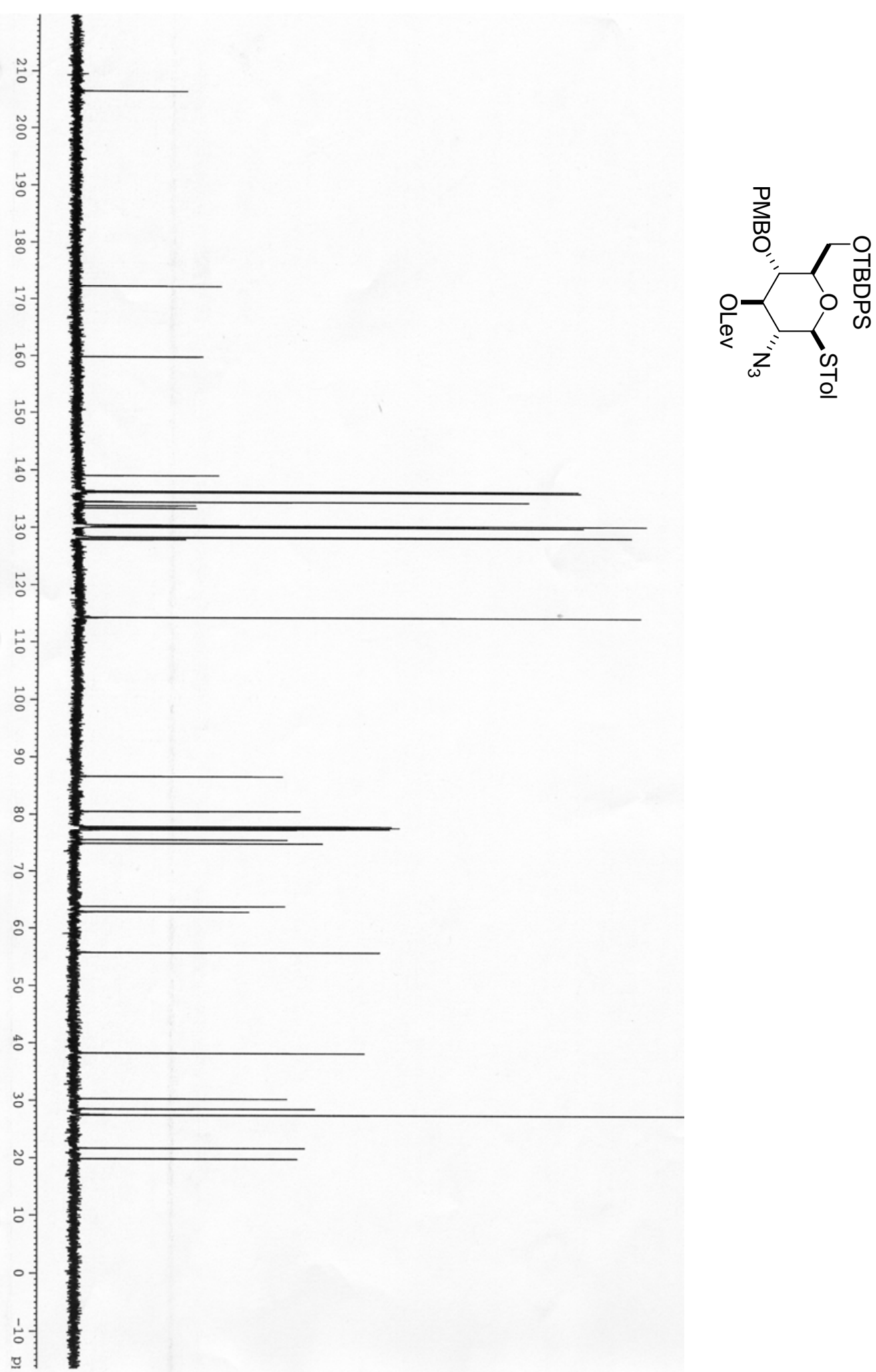

${ }^{13} \mathrm{C}$ NMR $\left(500 \mathrm{MHz}, \mathrm{CDCl}_{3}\right)$ Thioglycoside Donor 3 
Fan, R. H.; Achkar, J.; Hernández-Torres, J. M.; Wei, A.
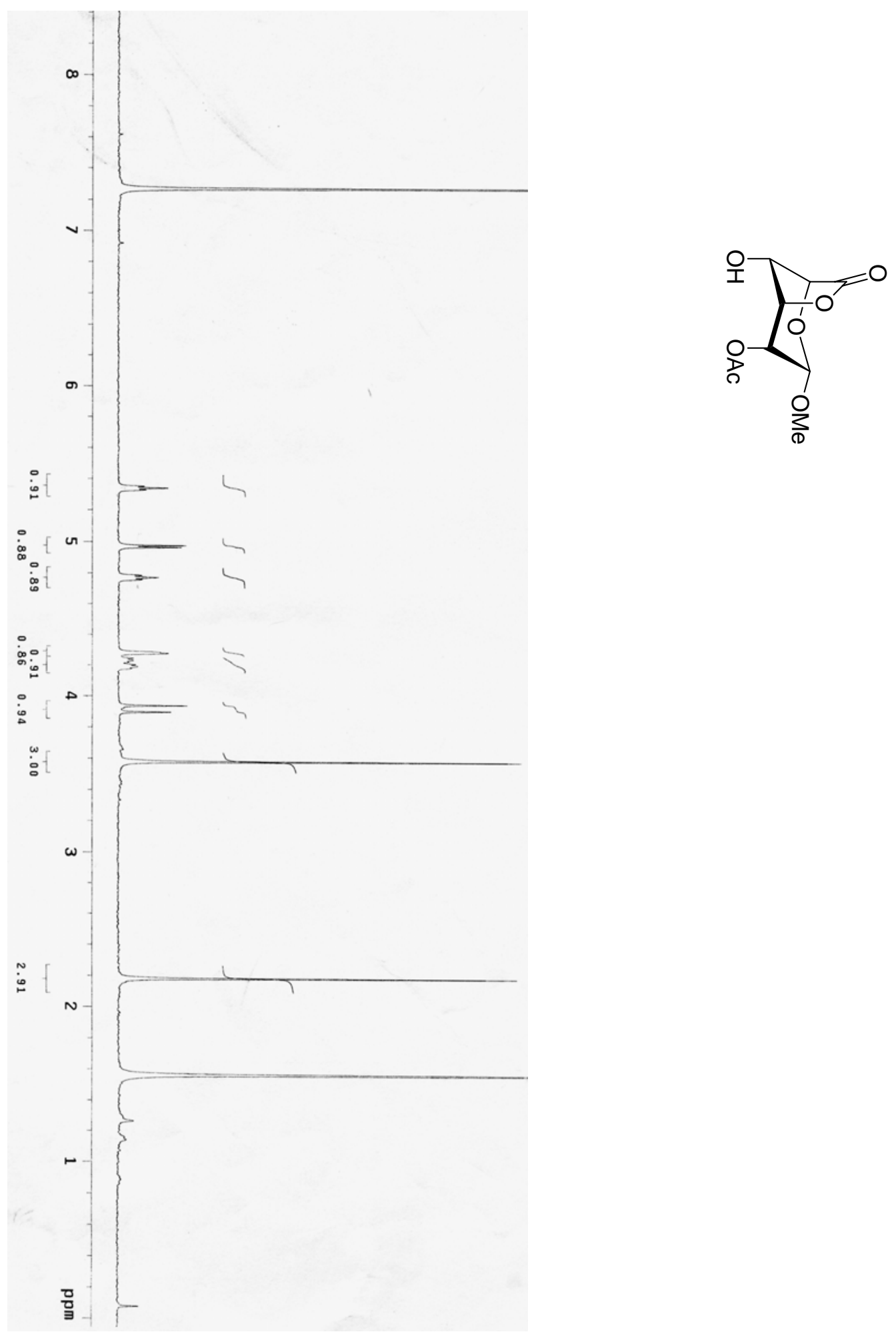

${ }^{1} \mathrm{H}$ NMR (300 MHz, $\mathrm{CDCl}_{3}$ ) Bicyclic Lactone Acceptor 4 
Fan, R. H.; Achkar, J.; Hernández-Torres, J. M.; Wei, A.
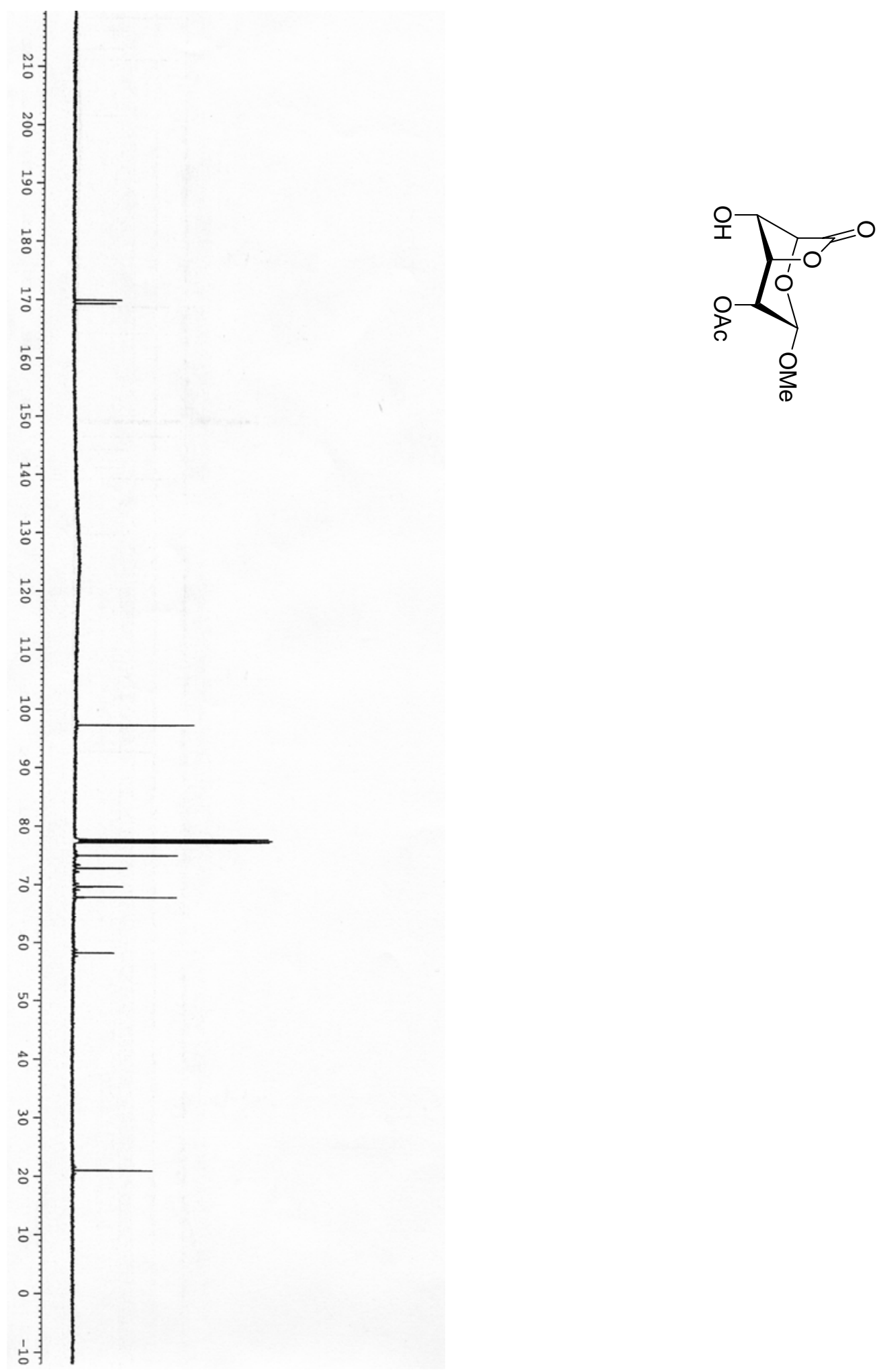

${ }^{13} \mathrm{C}$ NMR $\left(500 \mathrm{MHz}, \mathrm{CDCl}_{3}\right)$ Bicyclic Lactone Acceptor 4 
Fan, R. H.; Achkar, J.; Hernández-Torres, J. M.; Wei, A.
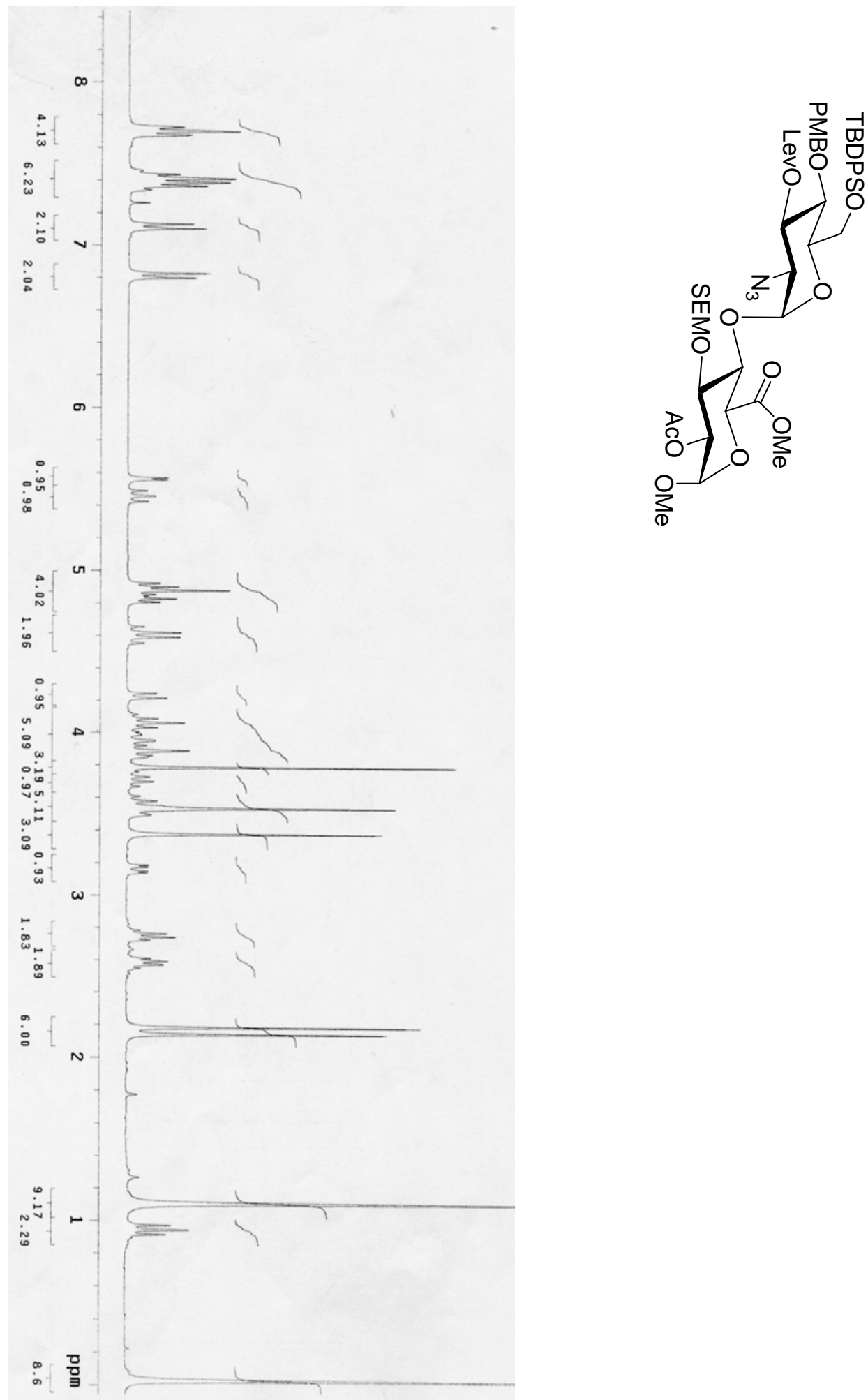

${ }^{1} \mathrm{H}$ NMR (300 MHz, $\left.\mathrm{CDCl}_{3}\right)$ Heparan Disaccharide 5 
Fan, R. H.; Achkar, J.; Hernández-Torres, J. M.; Wei, A.
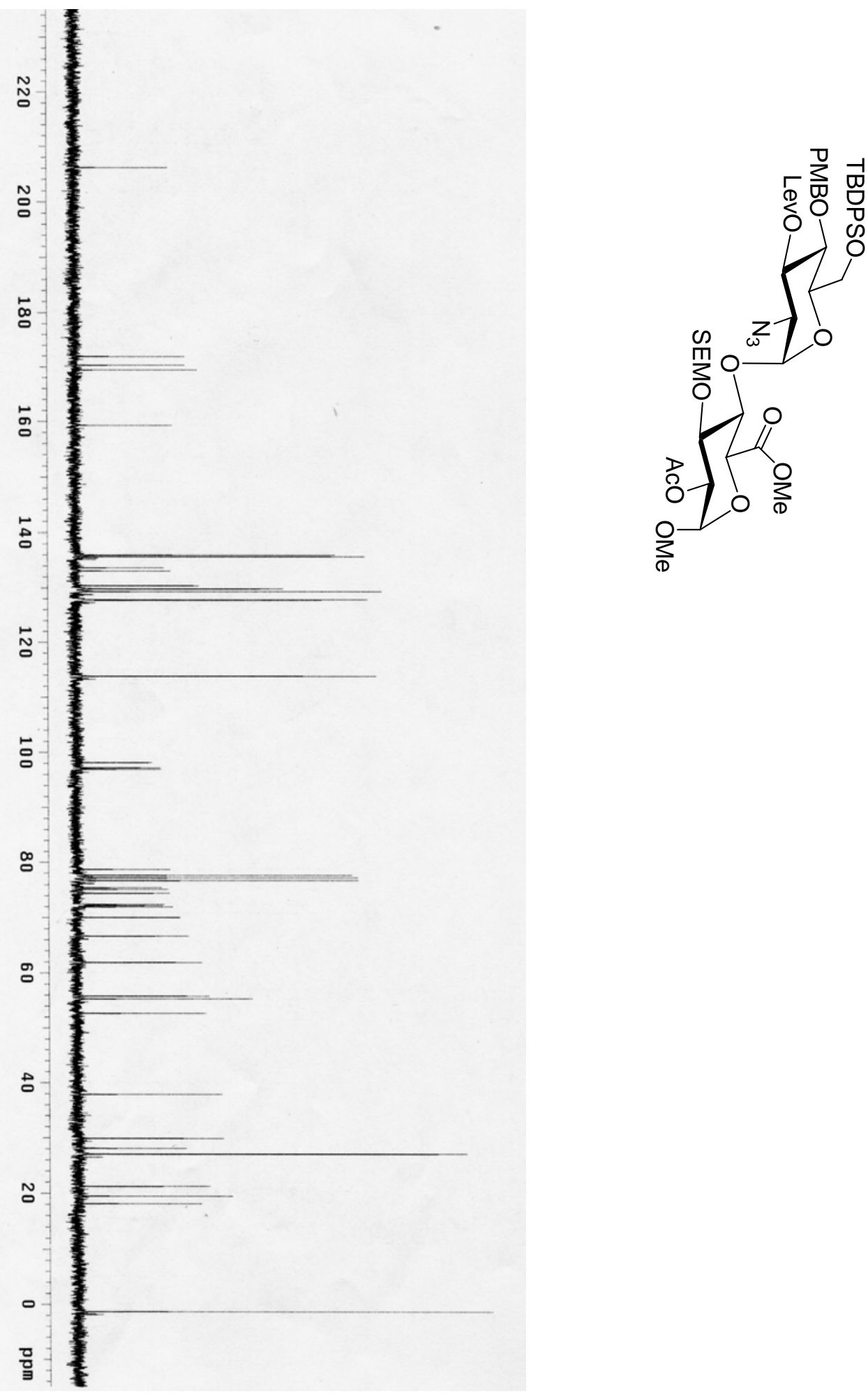

${ }^{13} \mathrm{C}$ NMR $\left(300 \mathrm{MHz}, \mathrm{CDCl}_{3}\right)$ Heparan Disaccharide 5 
Fan, R. H.; Achkar, J.; Hernández-Torres, J. M.; Wei, A.

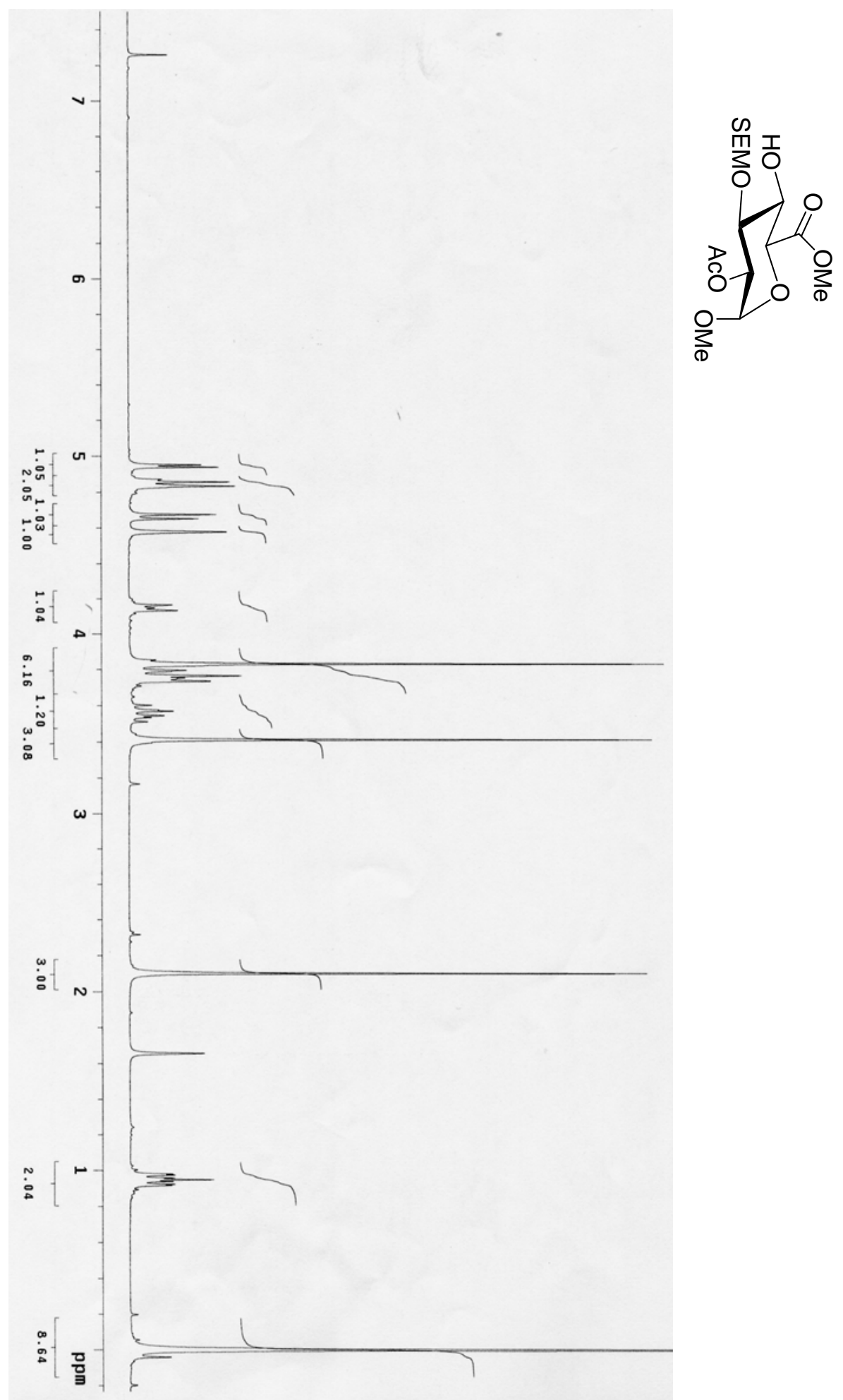

${ }^{1} \mathrm{H}$ NMR (300 MHz, $\left.\mathrm{CDCl}_{3}\right)$ Methyl Glucuronate 6 
Fan, R. H.; Achkar, J.; Hernández-Torres, J. M.; Wei, A.
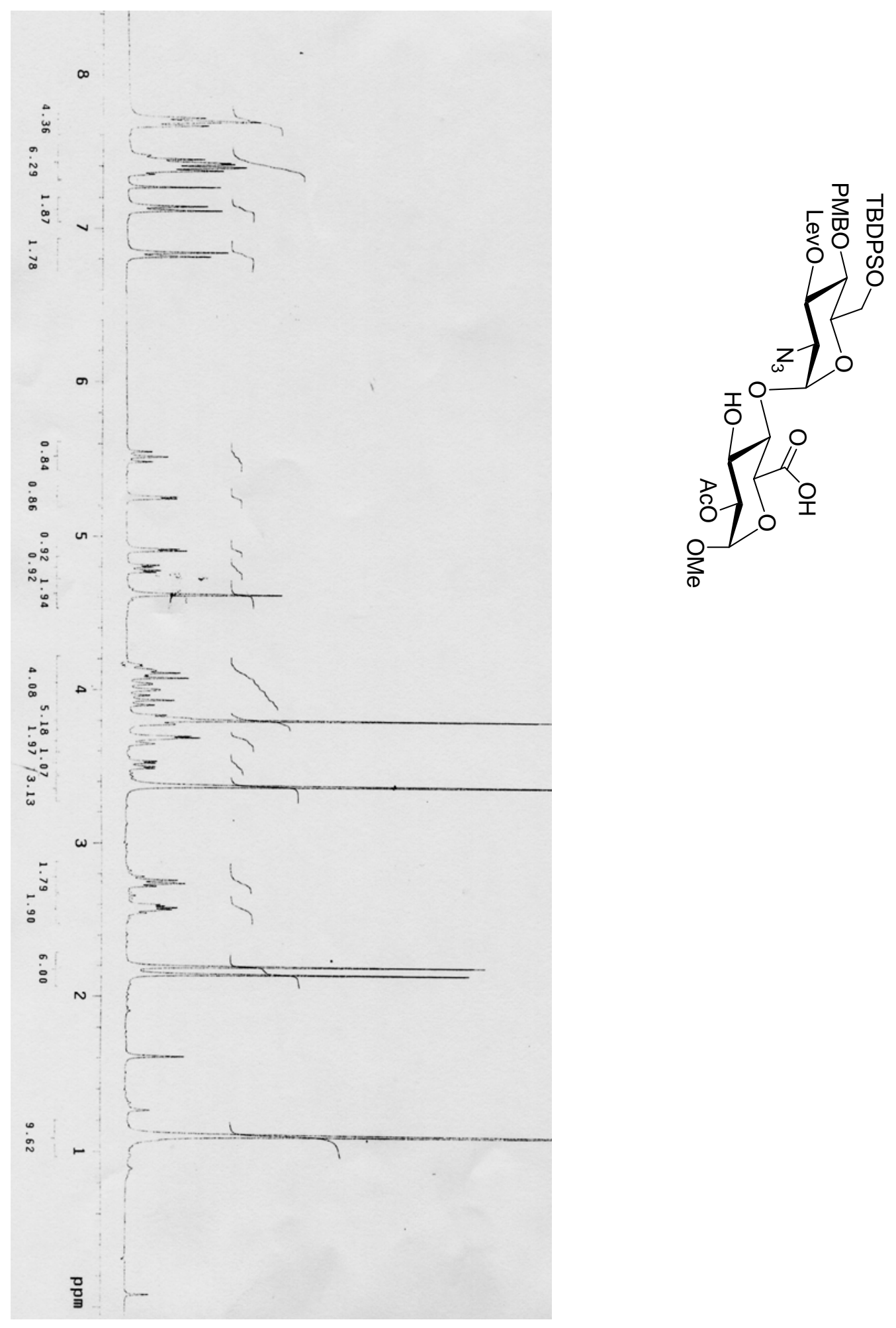

${ }^{1} \mathrm{H}$ NMR (300 MHz, $\mathrm{CDCl}_{3}$ ) Disaccharide Acid 7 
Fan, R. H.; Achkar, J.; Hernández-Torres, J. M.; Wei, A.

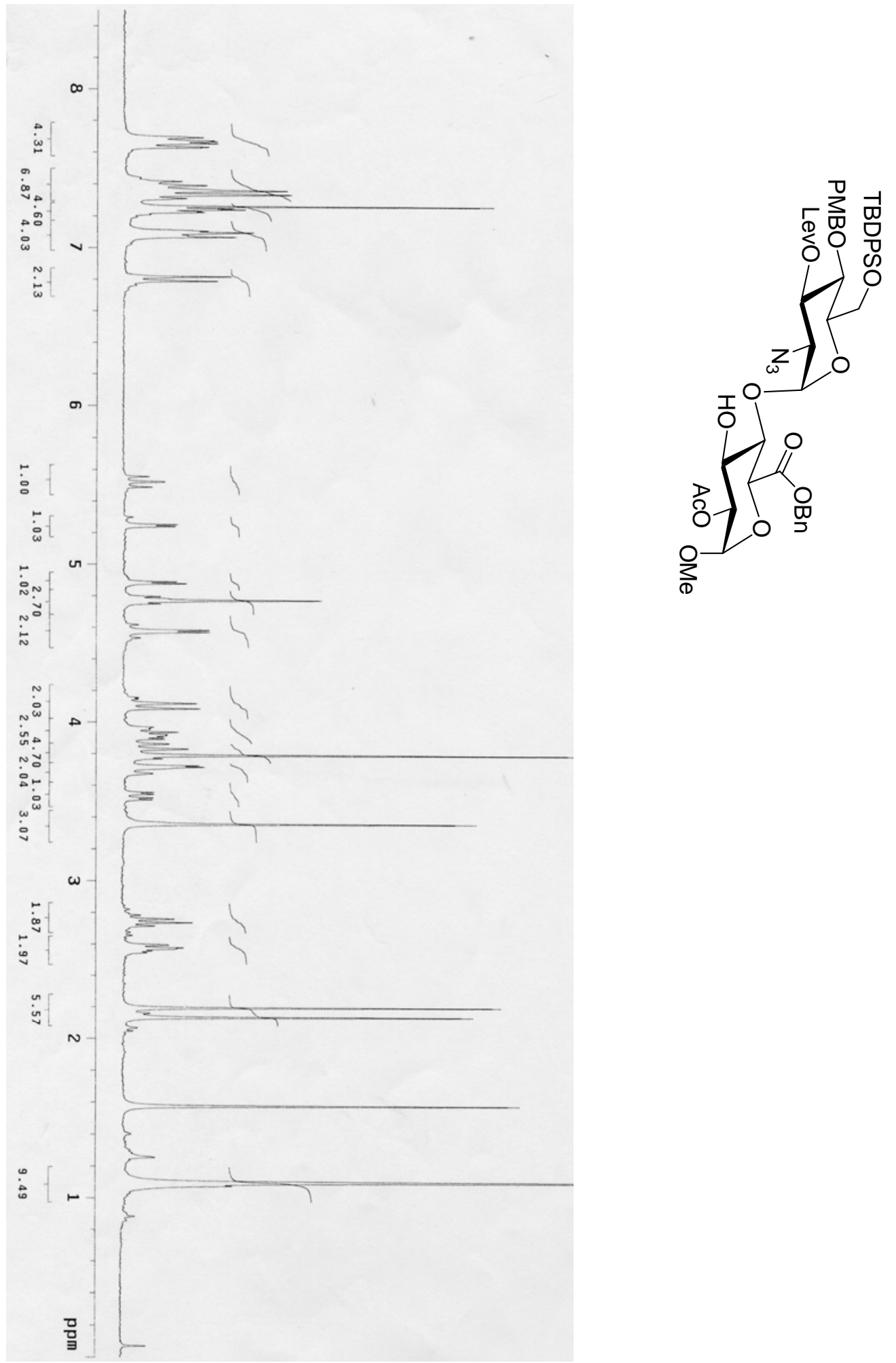

${ }^{1} \mathrm{H}$ NMR (300 MHz, $\mathrm{CDCl}_{3}$ ) Disaccharide Benzyl Ester 8 
Fan, R. H.; Achkar, J.; Hernández-Torres, J. M.; Wei, A.
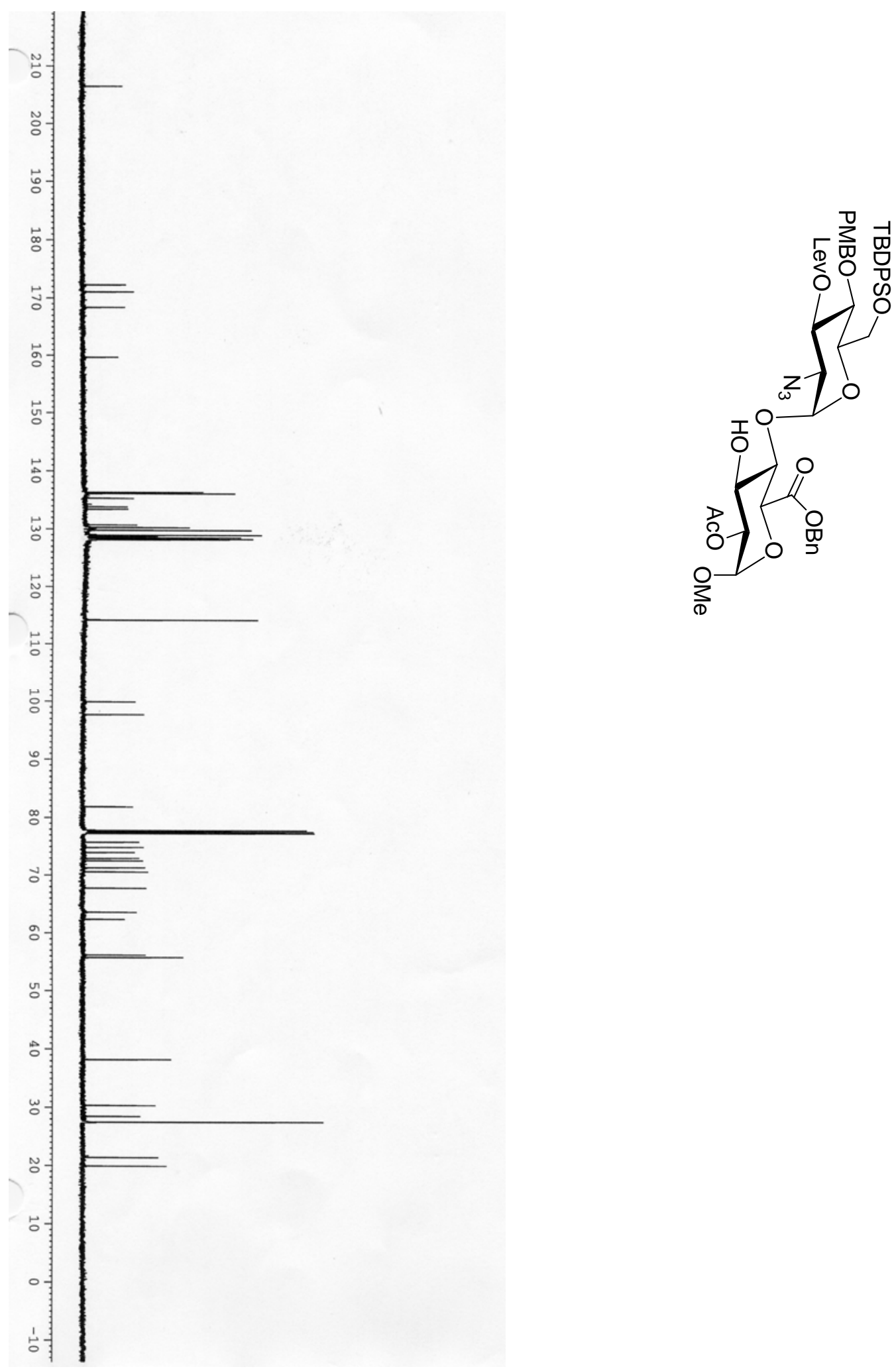

${ }^{13} \mathrm{C}$ NMR $\left(500 \mathrm{MHz}, \mathrm{CDCl}_{3}\right)$ Disaccharide Benzyl Ester 8 
Fan, R. H.; Achkar, J.; Hernández-Torres, J. M.; Wei, A.

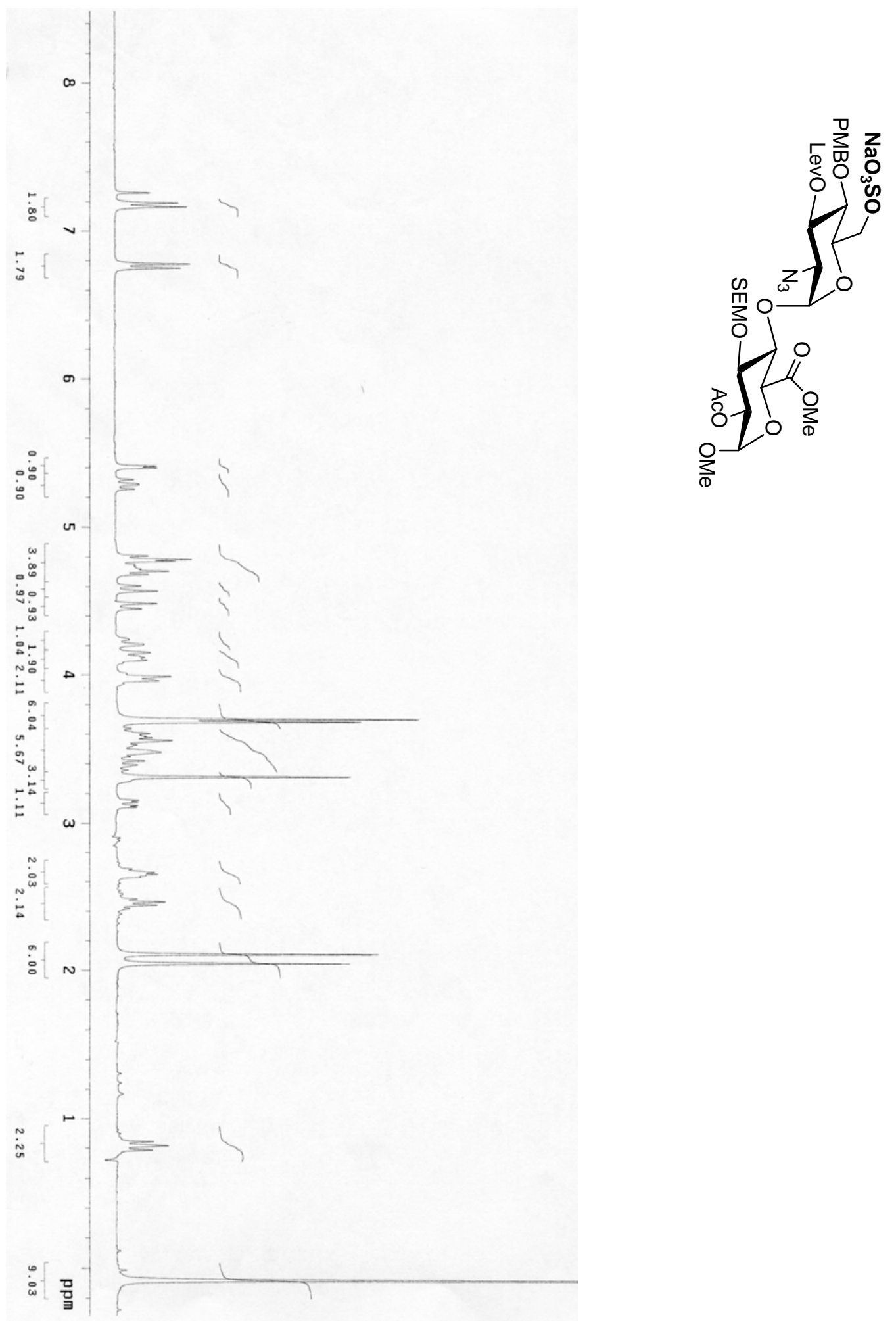

${ }^{1} \mathrm{H}$ NMR (300 MHz, 25\% $\mathrm{CD}_{3} \mathrm{OD}$ in $\mathrm{CDCl}_{3}$ ) Disaccharide 6'-O-Sulfate 9 
Fan, R. H.; Achkar, J.; Hernández-Torres, J. M.; Wei, A.
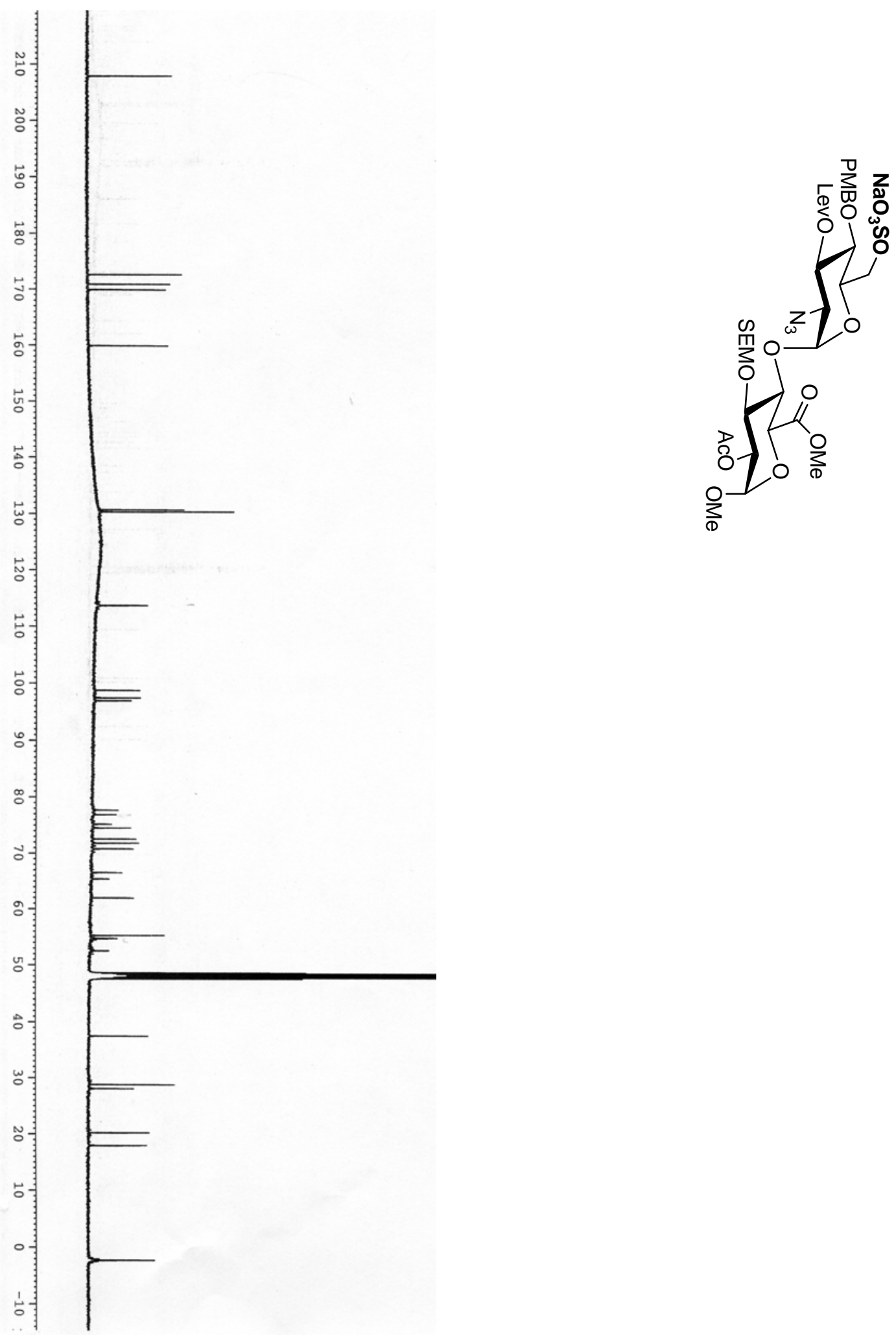

${ }^{13} \mathrm{C}$ NMR (500 MHz, $\mathrm{CD}_{3} \mathrm{OD}$ ) Disaccharide 6'-O-Sulfate 9 
Fan, R. H.; Achkar, J.; Hernández-Torres, J. M.; Wei, A.
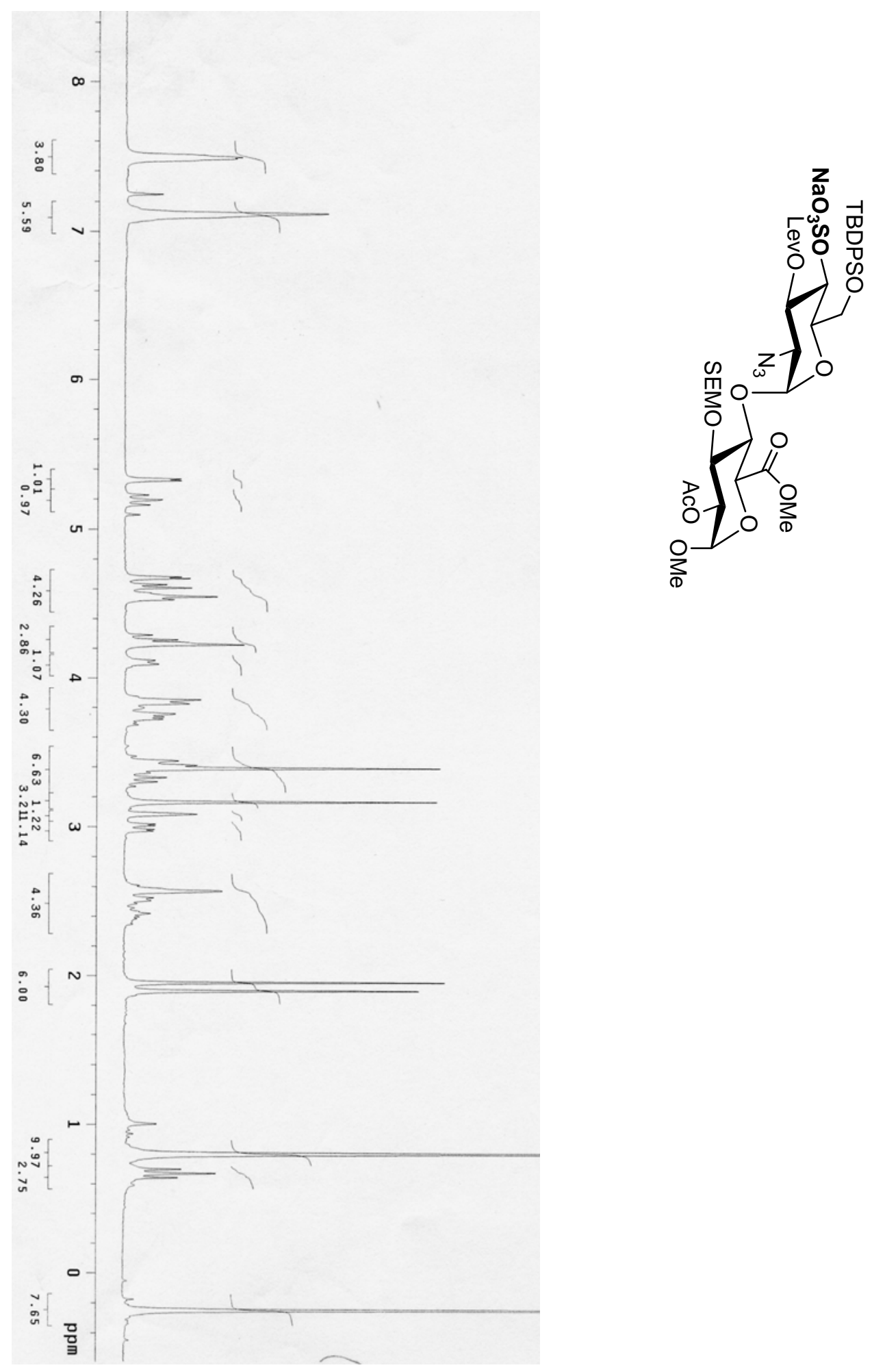

${ }^{1} \mathrm{H}$ NMR (300 MHz, 25\% $\mathrm{CD}_{3} \mathrm{OD}$ in $\mathrm{CDCl}_{3}$ ) Disaccharide 4'-O-Sulfate 10 
Fan, R. H.; Achkar, J.; Hernández-Torres, J. M.; Wei, A.

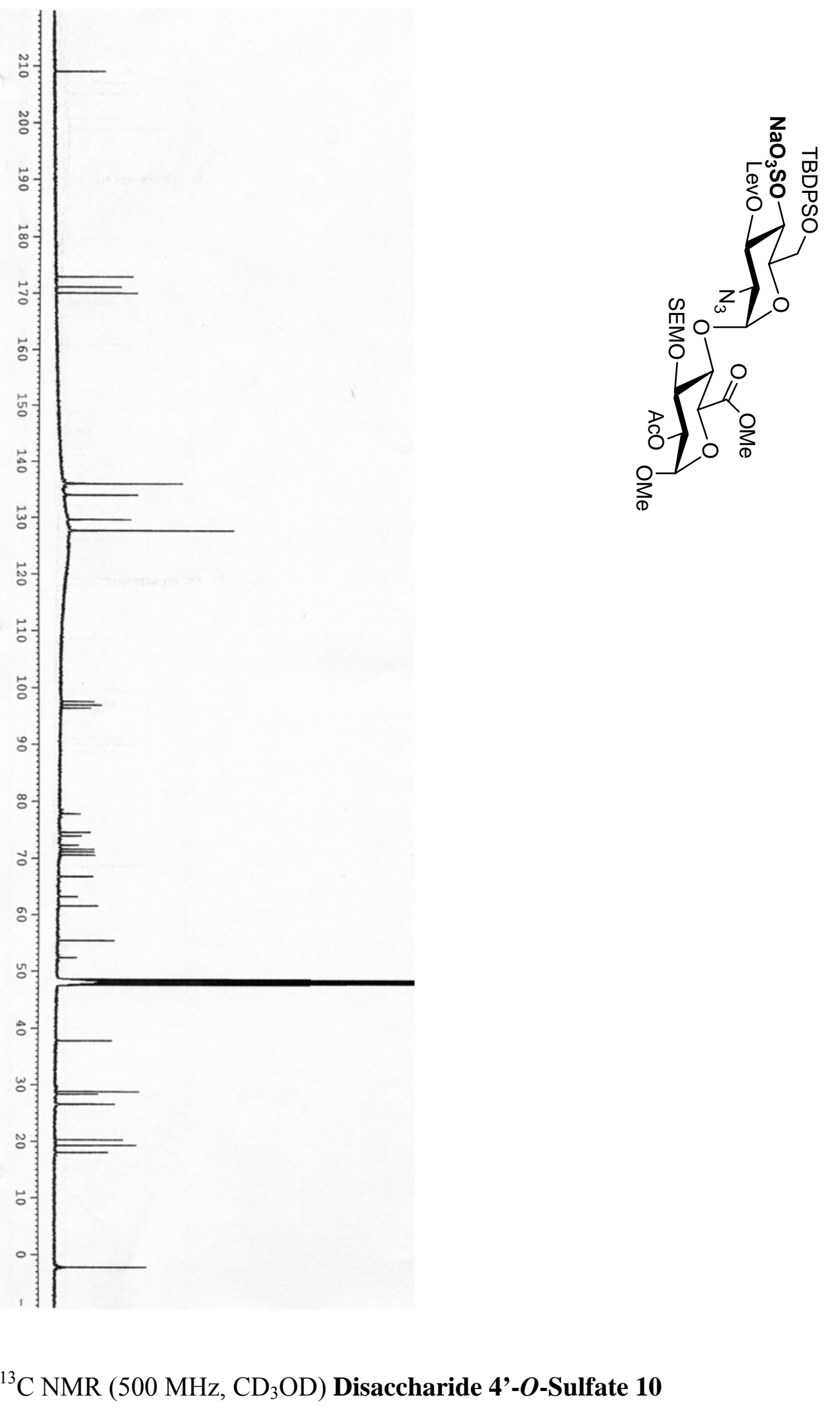


Fan, R. H.; Achkar, J.; Hernández-Torres, J. M.; Wei, A.
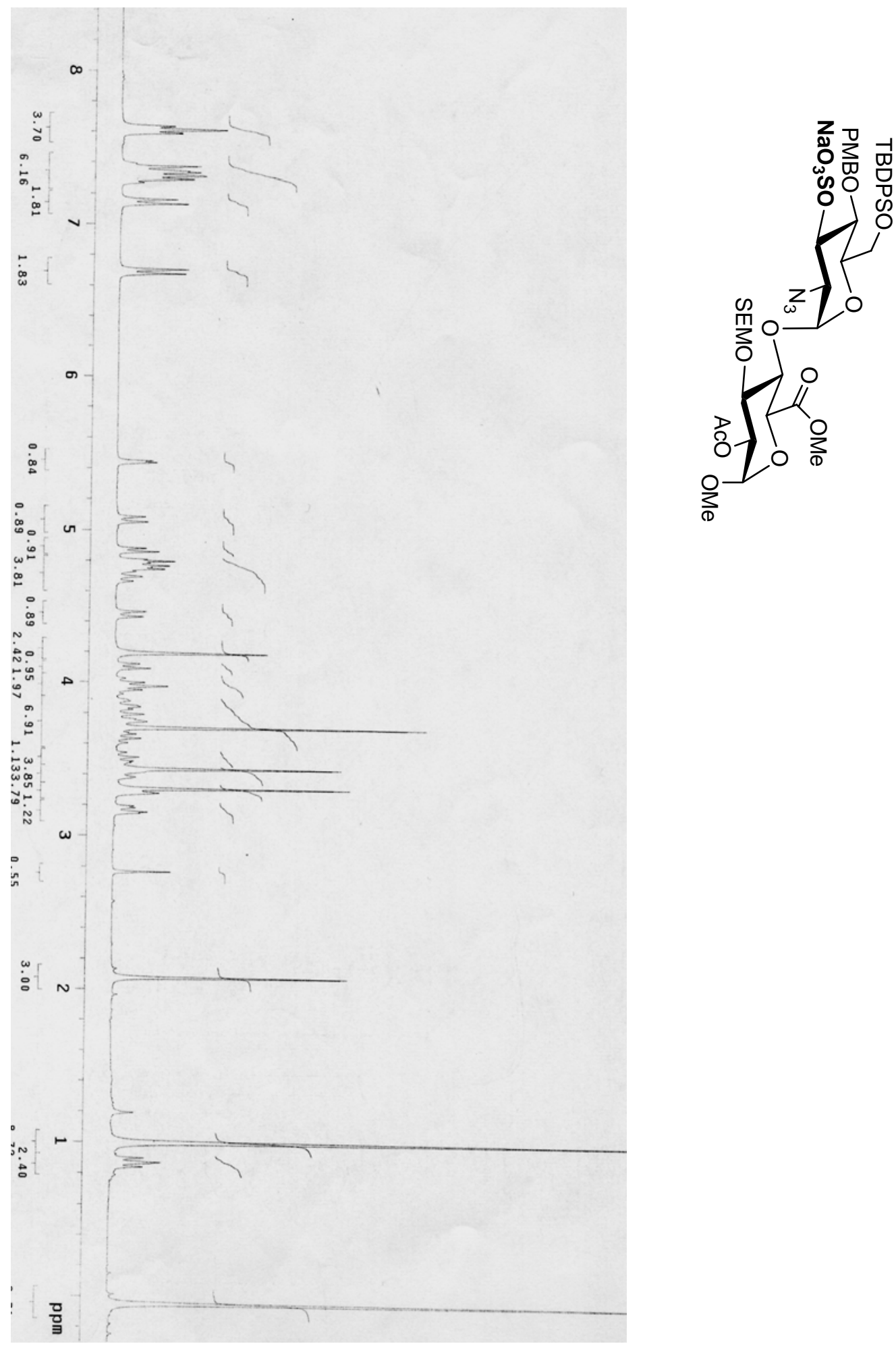

${ }^{1} \mathrm{H}$ NMR (300 MHz, 25\% $\mathrm{CD}_{3} \mathrm{OD}$ in $\mathrm{CDCl}_{3}$ ) Disaccharide 3'-O-Sulfate 11 
Fan, R. H.; Achkar, J.; Hernández-Torres, J. M.; Wei, A.
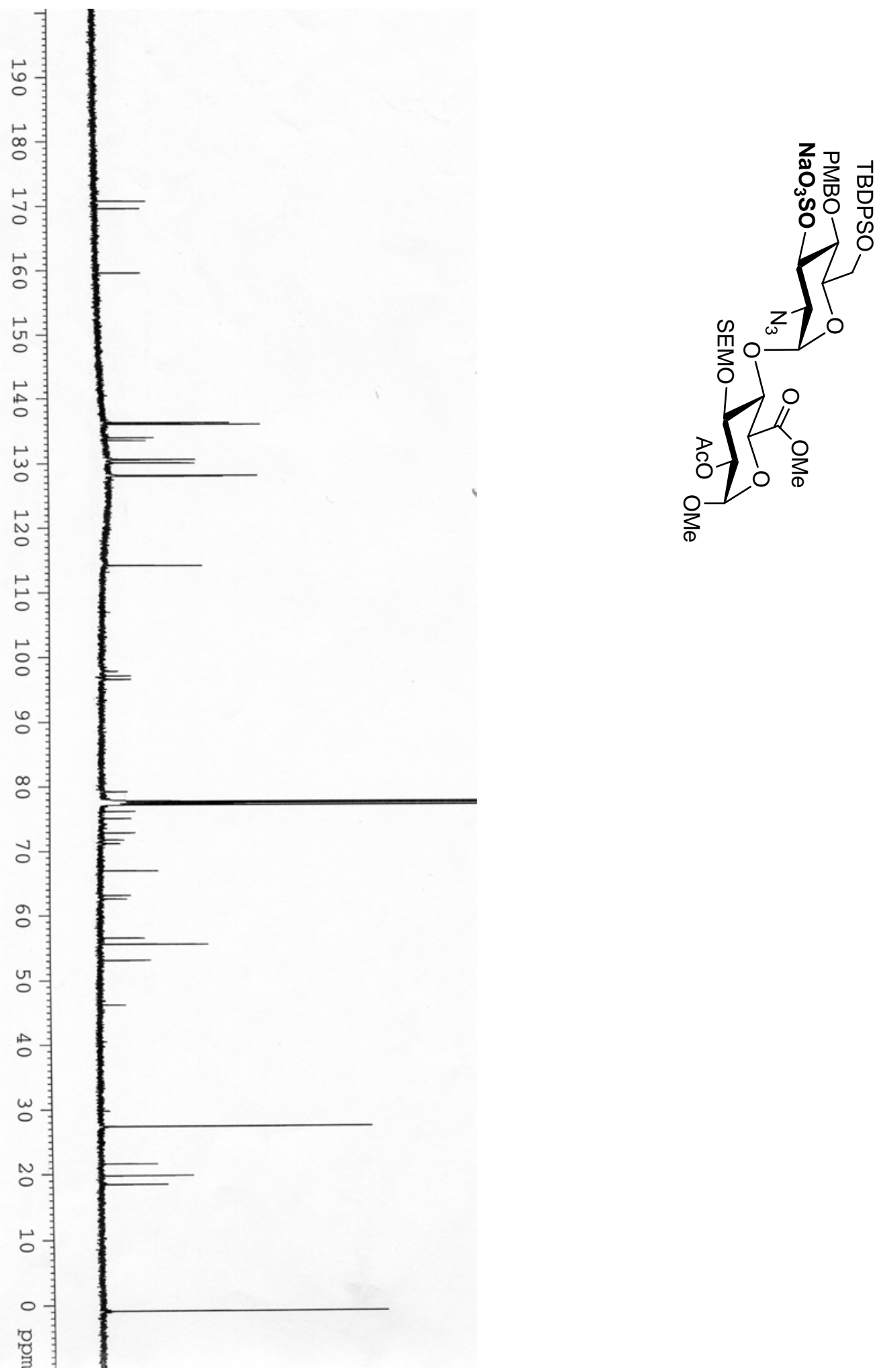

${ }^{13} \mathrm{C}$ NMR (500 MHz, $\mathrm{CDCl}_{3}$ ) Disaccharide 3'-O-Sulfate 11 
Fan, R. H.; Achkar, J.; Hernández-Torres, J. M.; Wei, A.

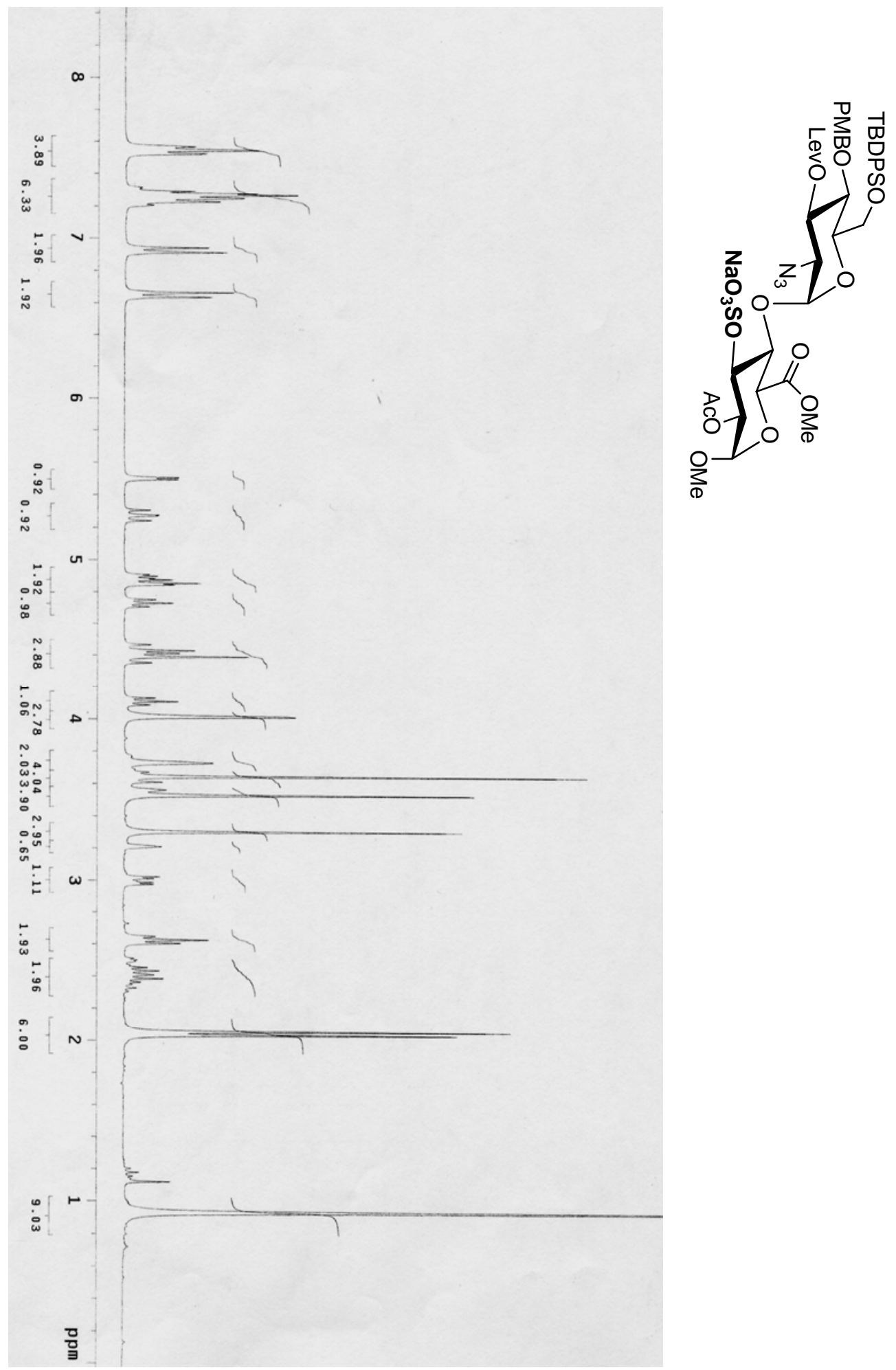

${ }^{1} \mathrm{H}$ NMR (300 MHz, 25\% $\mathrm{CD}_{3} \mathrm{OD}$ in $\mathrm{CDCl}_{3}$ ) Disaccharide 3-O-Sulfate 12 
Fan, R. H.; Achkar, J.; Hernández-Torres, J. M.; Wei, A.
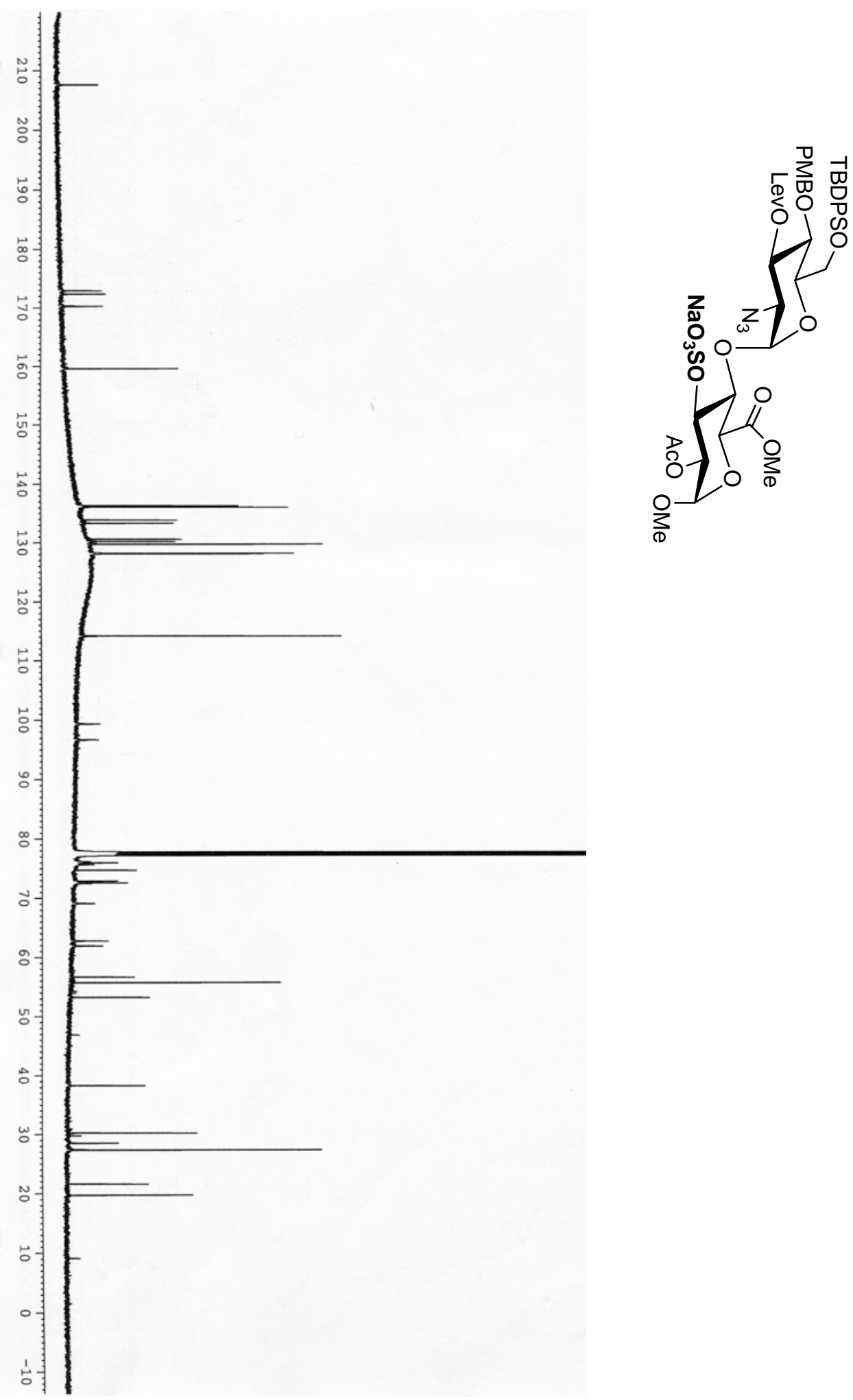

${ }^{13} \mathrm{C}$ NMR (500 MHz, $\mathrm{CDCl}_{3}$ ) Disaccharide 3-O-Sulfate 12 
Fan, R. H.; Achkar, J.; Hernández-Torres, J. M.; Wei, A.
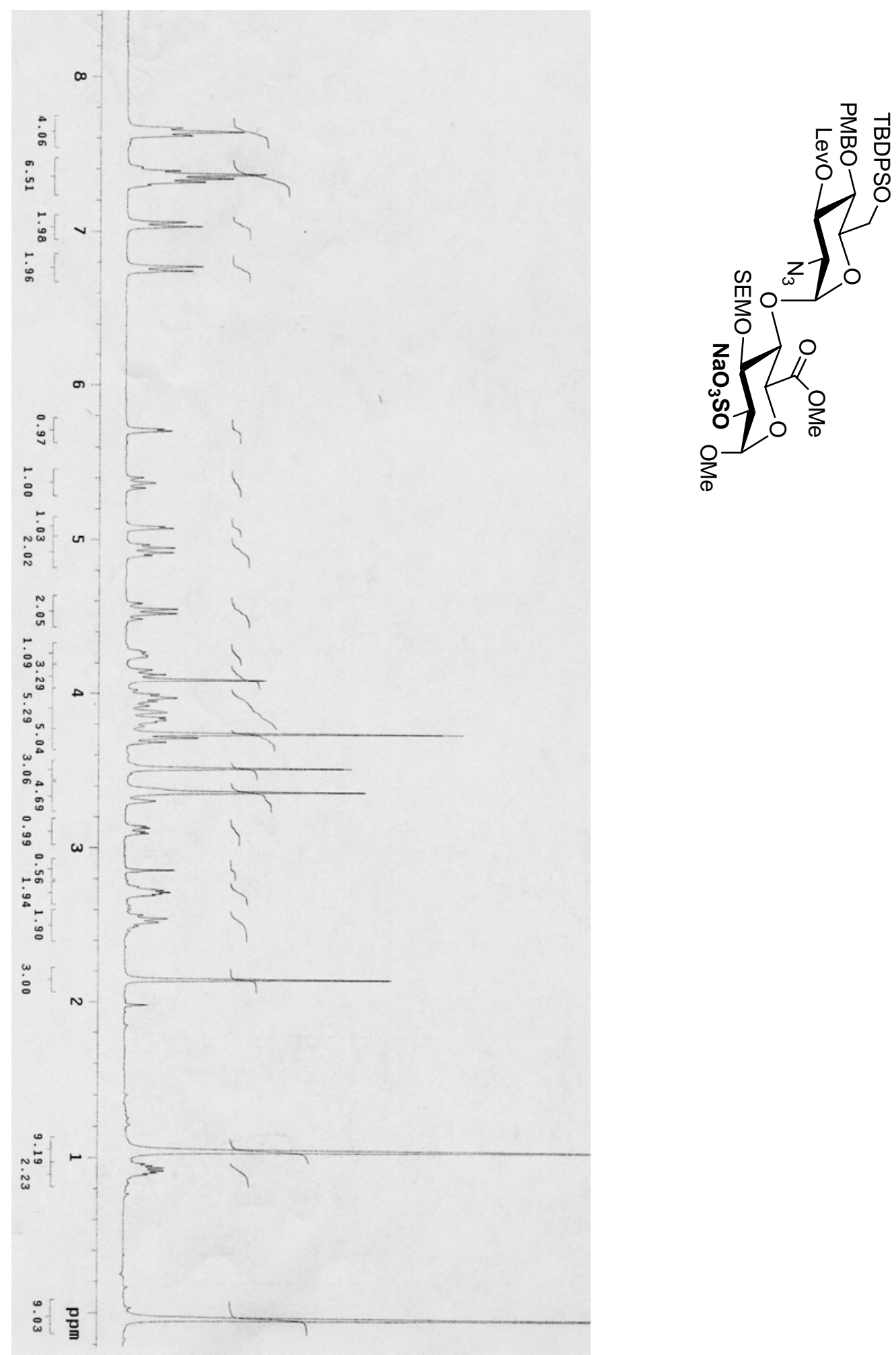

${ }^{1} \mathrm{H}$ NMR (300 MHz, 25\% $\mathrm{CD}_{3} \mathrm{OD}$ in $\mathrm{CDCl}_{3}$ ) Disaccharide 2-O-Sulfate 13 
Fan, R. H.; Achkar, J.; Hernández-Torres, J. M.; Wei, A.

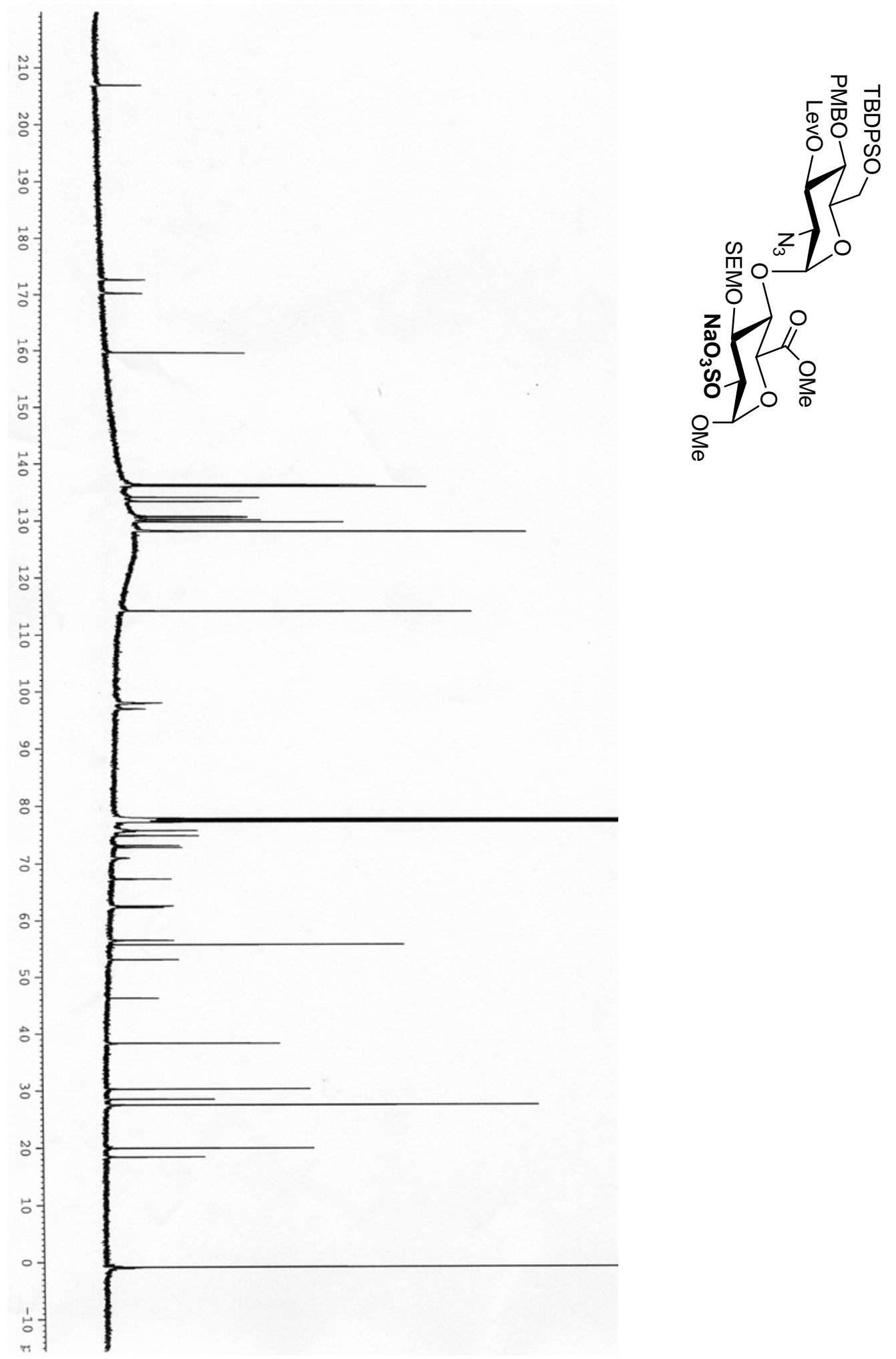

${ }^{13} \mathrm{C}$ NMR (500 MHz, $\mathrm{CDCl}_{3}$ ) Disaccharide 2-O-Sulfate 13 
Fan, R. H.; Achkar, J.; Hernández-Torres, J. M.; Wei, A.

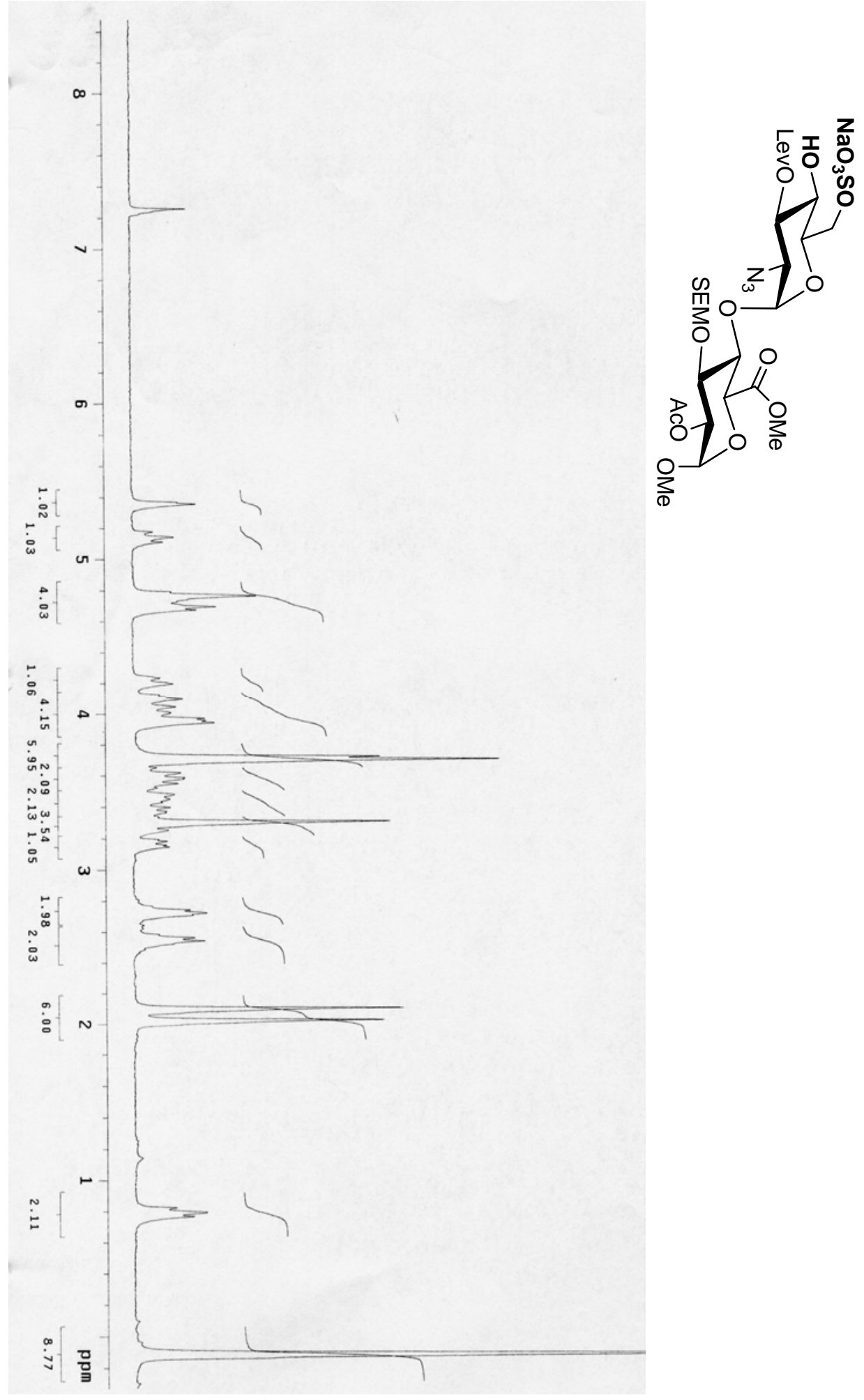

${ }^{1} \mathrm{H}$ NMR (300 MHz, 25\% $\mathrm{CD}_{3} \mathrm{OD}$ in $\mathrm{CDCl}_{3}$ ) Disaccharide 6'-O-Sulfate-4'-OH 14 
Fan, R. H.; Achkar, J.; Hernández-Torres, J. M.; Wei, A.
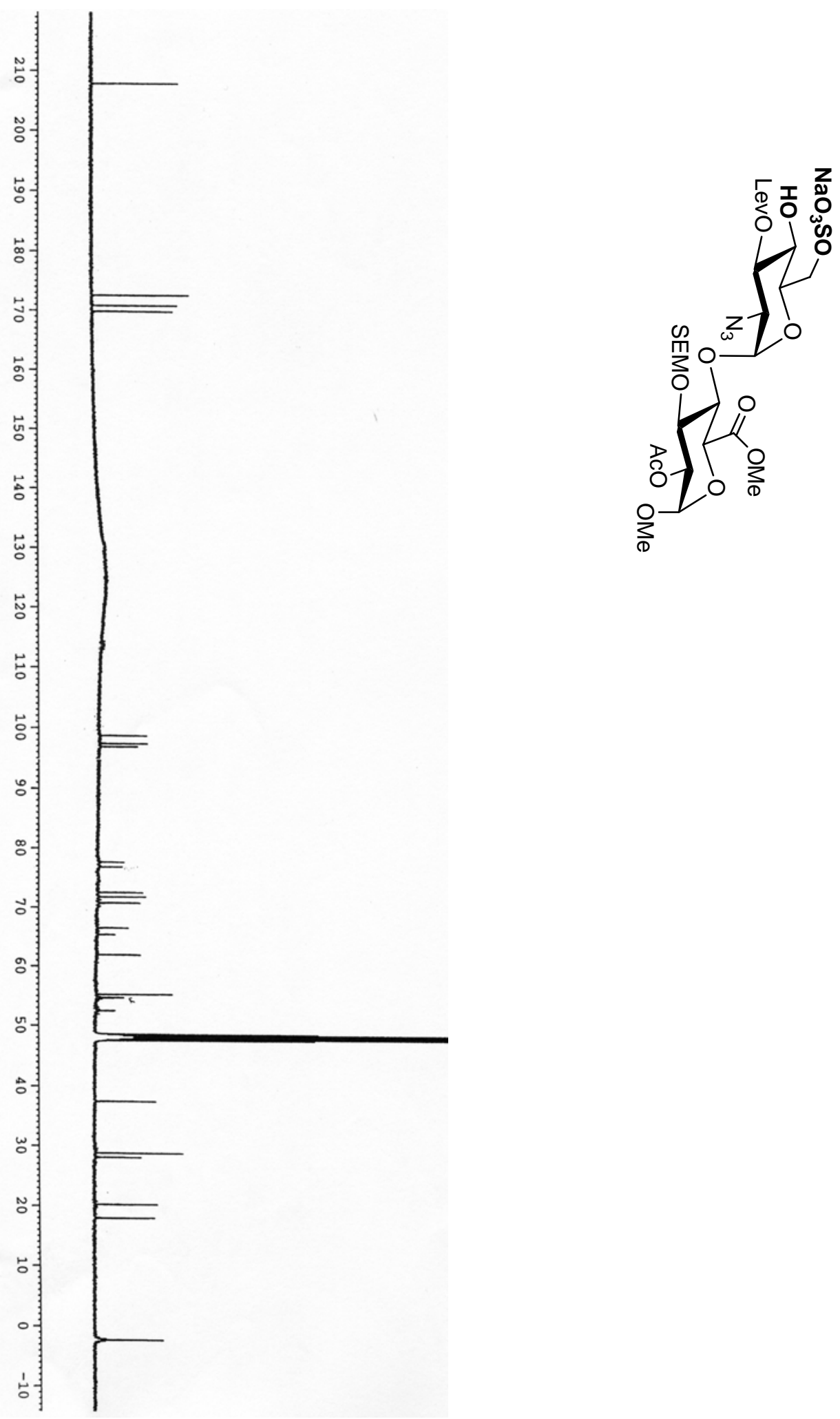

${ }^{13} \mathrm{C}$ NMR (500 MHz, CD $\mathrm{CD}_{3} \mathrm{OD}$ ) Disaccharide 6'-O-Sulfate-4'-OH 14 
Fan, R. H.; Achkar, J.; Hernández-Torres, J. M.; Wei, A.
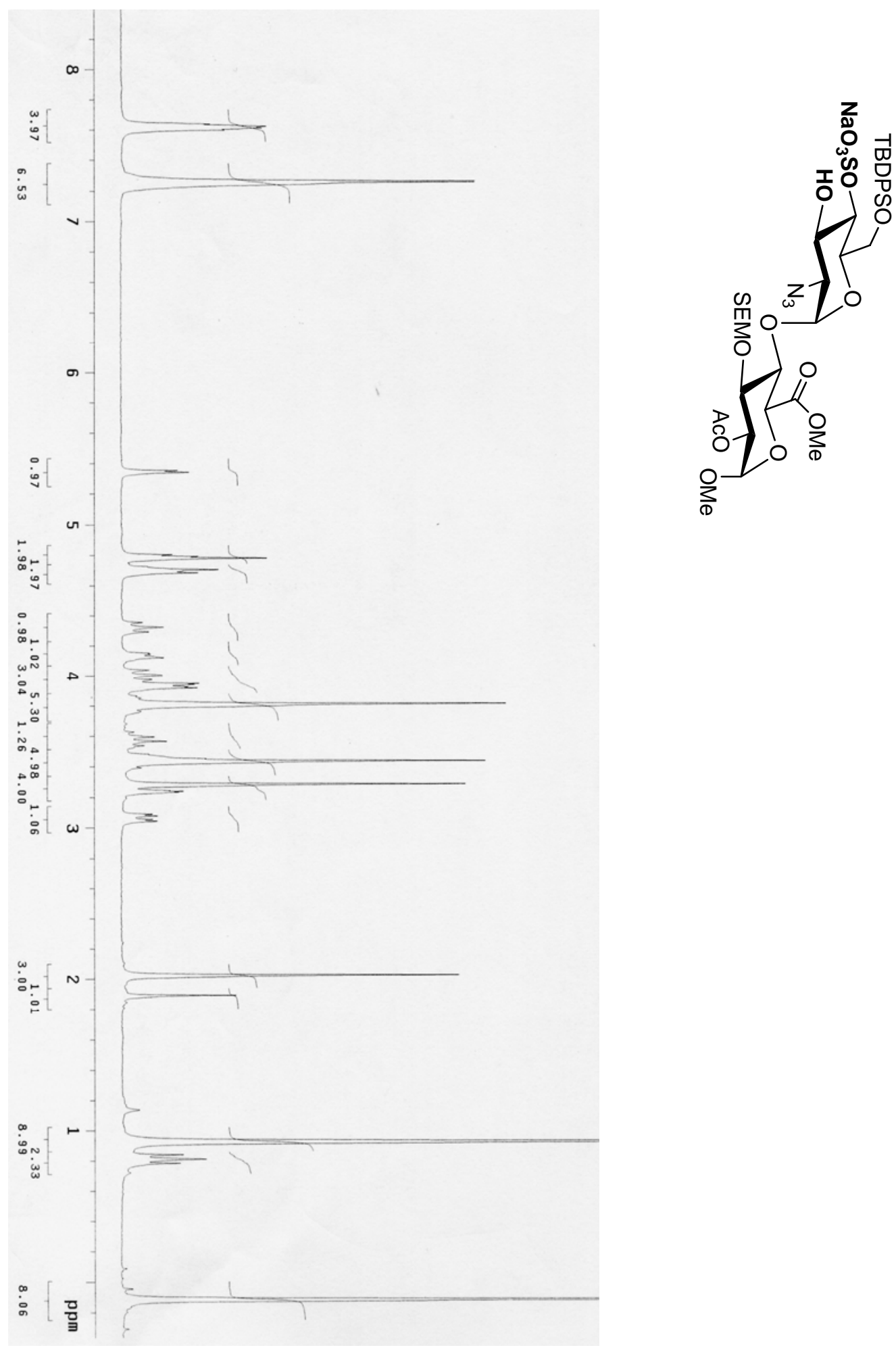

${ }^{1} \mathrm{H}$ NMR (300 MHz, 25\% $\mathrm{CD}_{3} \mathrm{OD}$ in $\mathrm{CDCl}_{3}$ ) Disaccharide 4'-O-Sulfate-3'-OH 15 
Fan, R. H.; Achkar, J.; Hernández-Torres, J. M.; Wei, A.
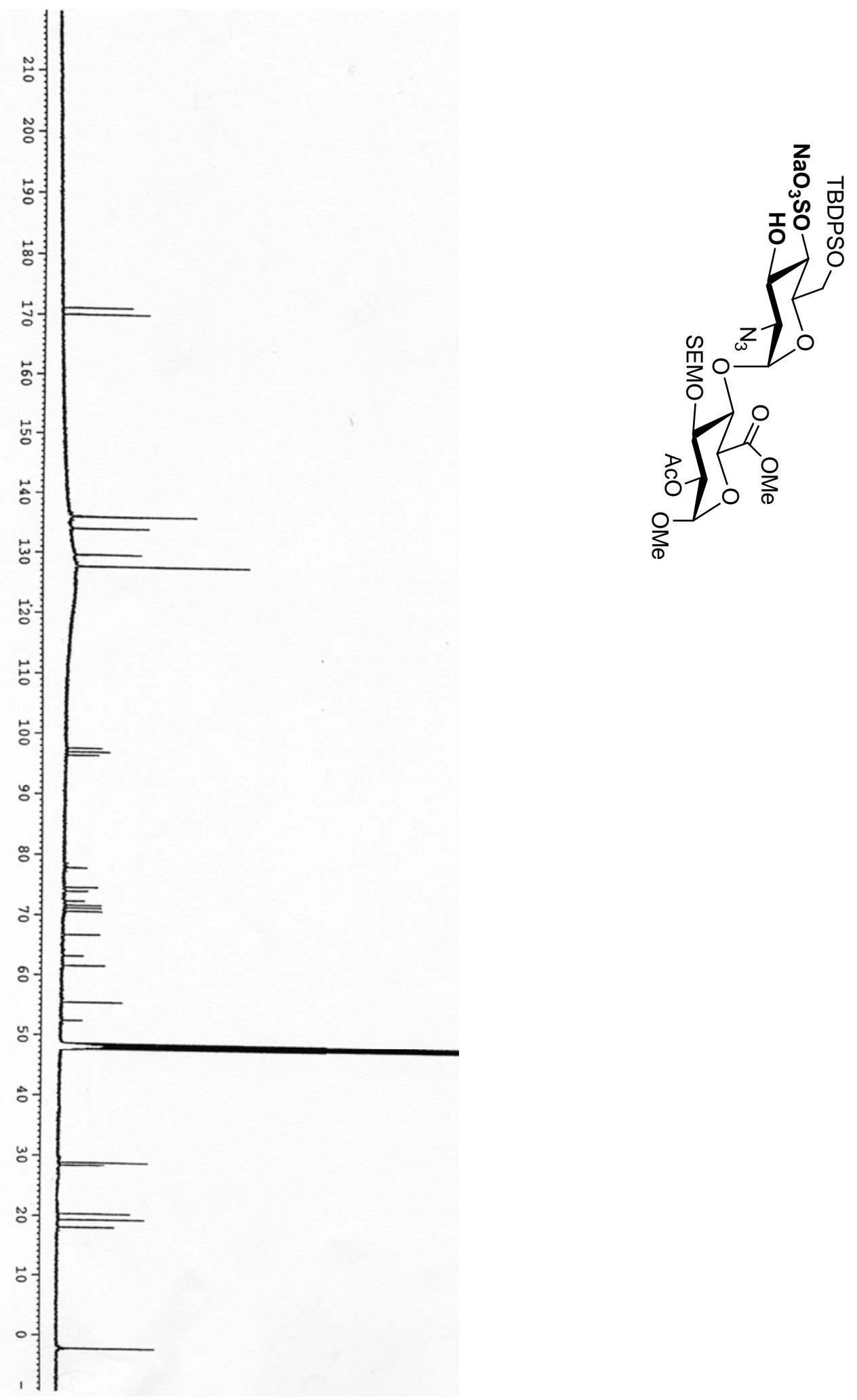

${ }^{13} \mathrm{C}$ NMR (500 MHz, CD $\mathrm{OD}$ ) Disaccharide 4'-O-Sulfate-3’-OH 15 
Fan, R. H.; Achkar, J.; Hernández-Torres, J. M.; Wei, A.

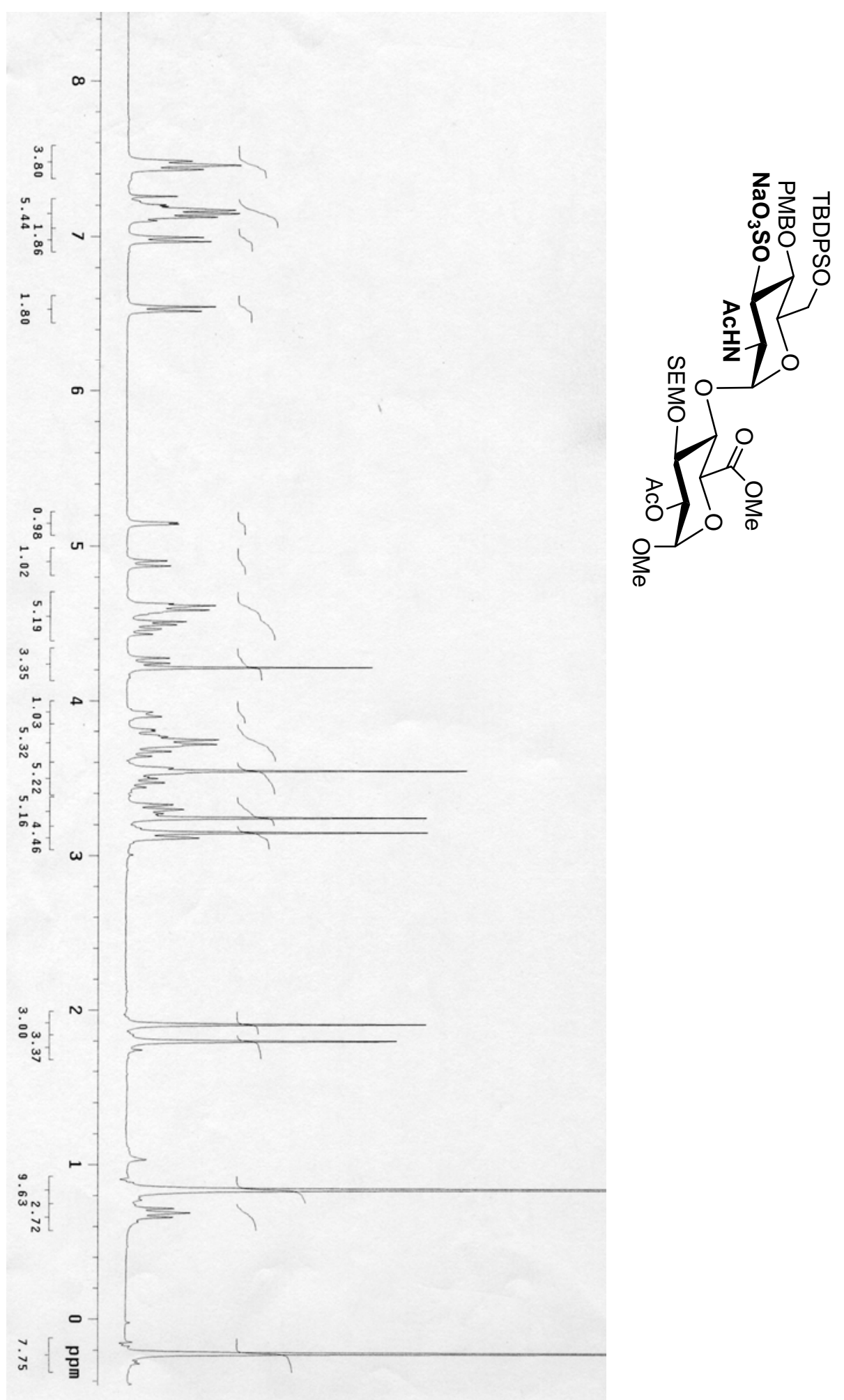

${ }^{1} \mathrm{H}$ NMR (300 MHz, 25\% $\mathrm{CD}_{3} \mathrm{OD}$ in $\mathrm{CDCl}_{3}$ ) Disaccharide 3'-O-Sulfate-2'-NHAc 16 


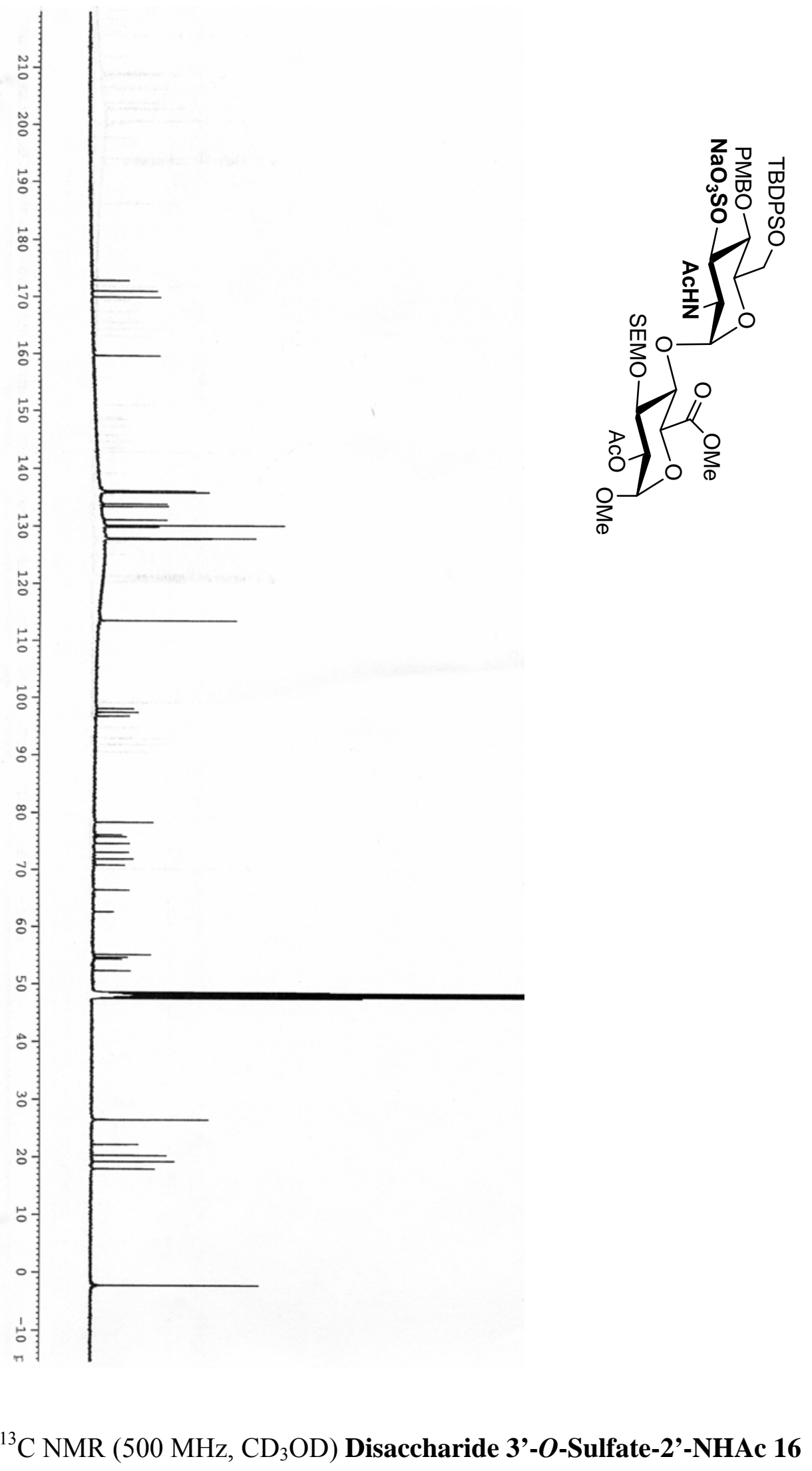


Fan, R. H.; Achkar, J.; Hernández-Torres, J. M.; Wei, A.

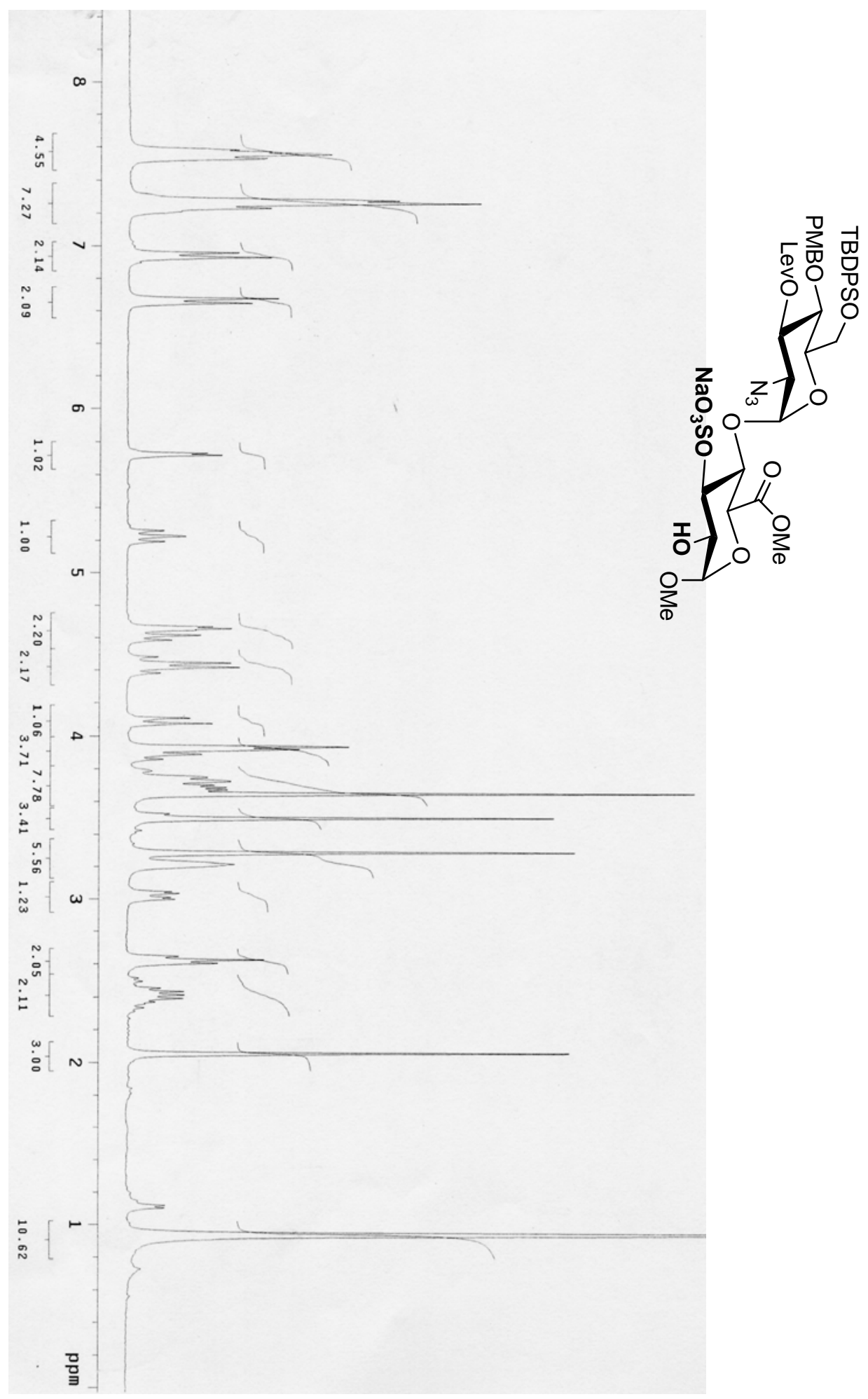

${ }^{1} \mathrm{H}$ NMR (300 MHz, 25\% $\mathrm{CD}_{3} \mathrm{OD}$ in $\mathrm{CDCl}_{3}$ ) Disaccharide 3-O-Sulfate-2-OH 17 
Fan, R. H.; Achkar, J.; Hernández-Torres, J. M.; Wei, A.
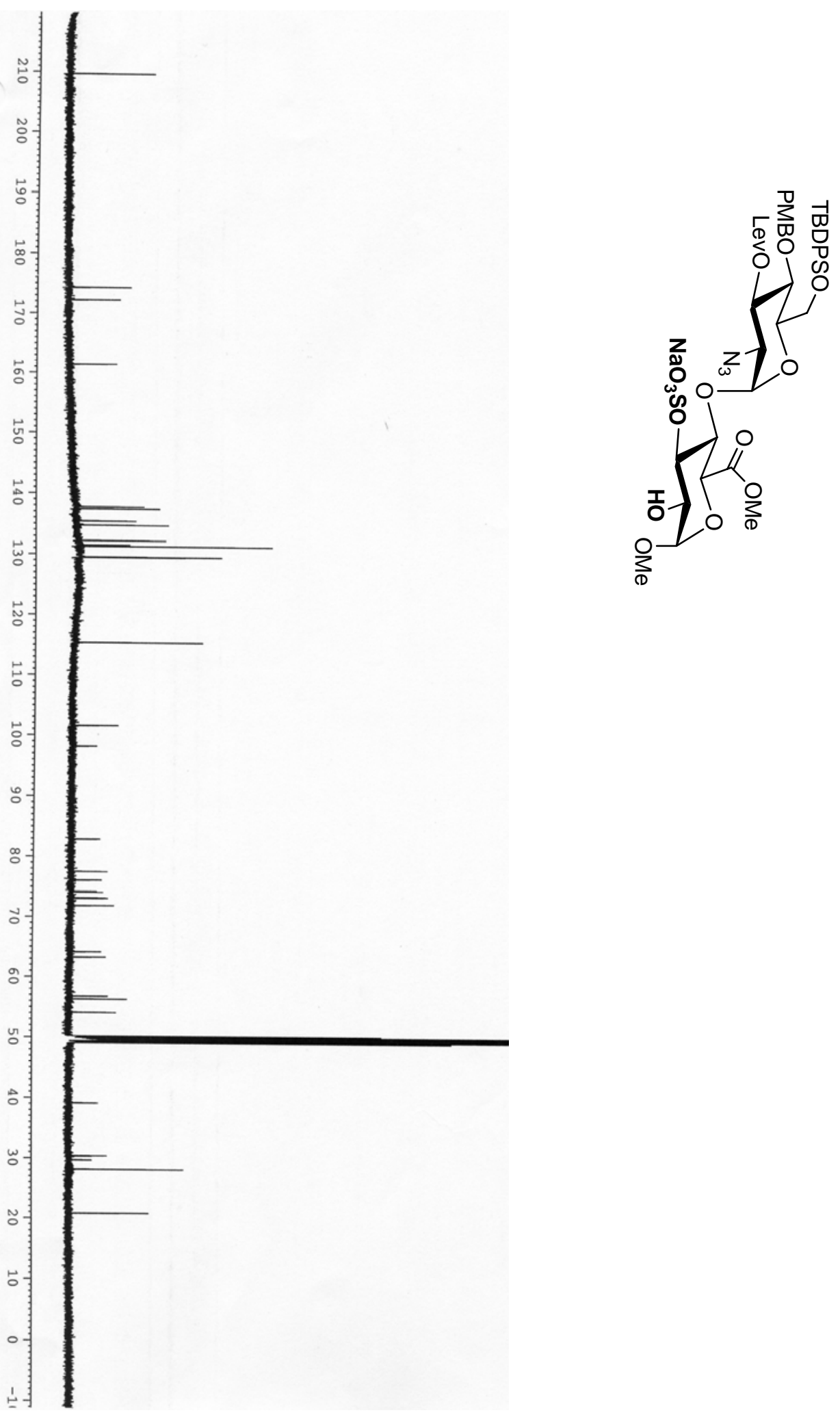

${ }^{13} \mathrm{C}$ NMR (500 MHz, CD $\mathrm{OD}$ ) Disaccharide 3-O-Sulfate-2-OH 17 
Fan, R. H.; Achkar, J.; Hernández-Torres, J. M.; Wei, A.

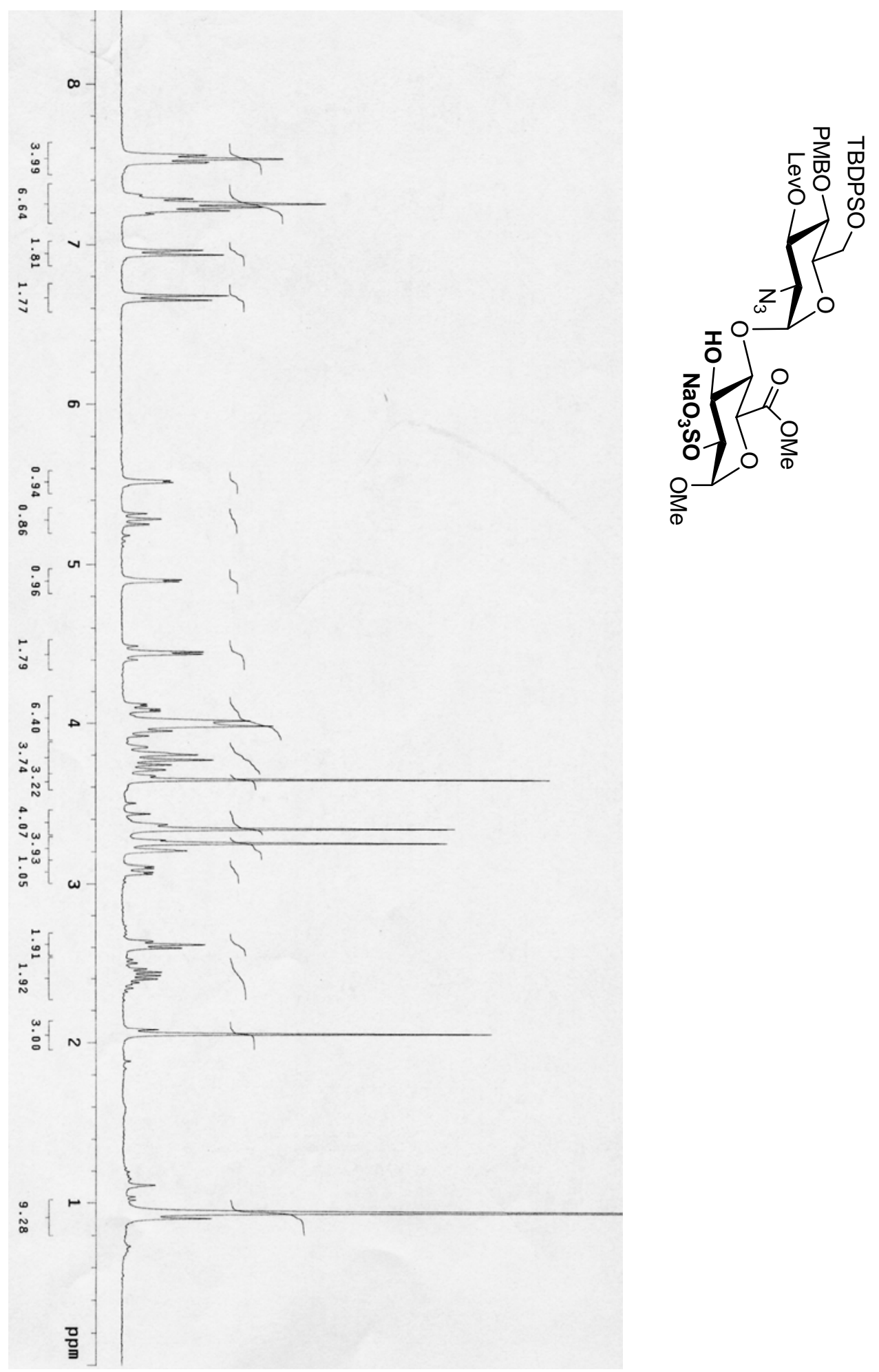

${ }^{1} \mathrm{H}$ NMR (300 MHz, 25\% $\mathrm{CD}_{3} \mathrm{OD}$ in $\mathrm{CDCl}_{3}$ ) Disaccharide 2-O-Sulfate-3-OH 18 
Fan, R. H.; Achkar, J.; Hernández-Torres, J. M.; Wei, A.
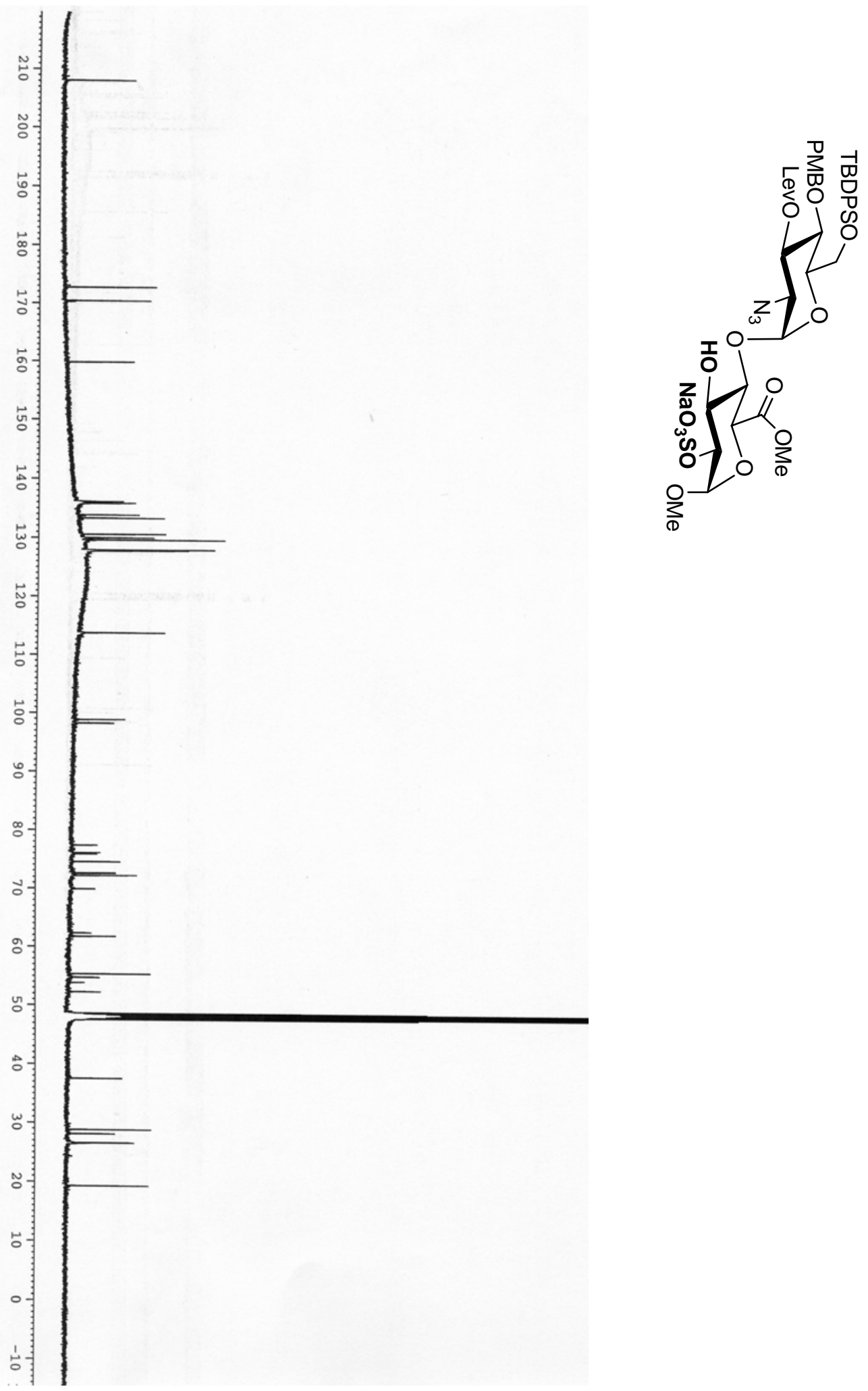

${ }^{13} \mathrm{C}$ NMR (500 MHz, CD $\mathrm{OD}$ ) Disaccharide 2-O-Sulfate-3-OH 18 
Fan, R. H.; Achkar, J.; Hernández-Torres, J. M.; Wei, A.

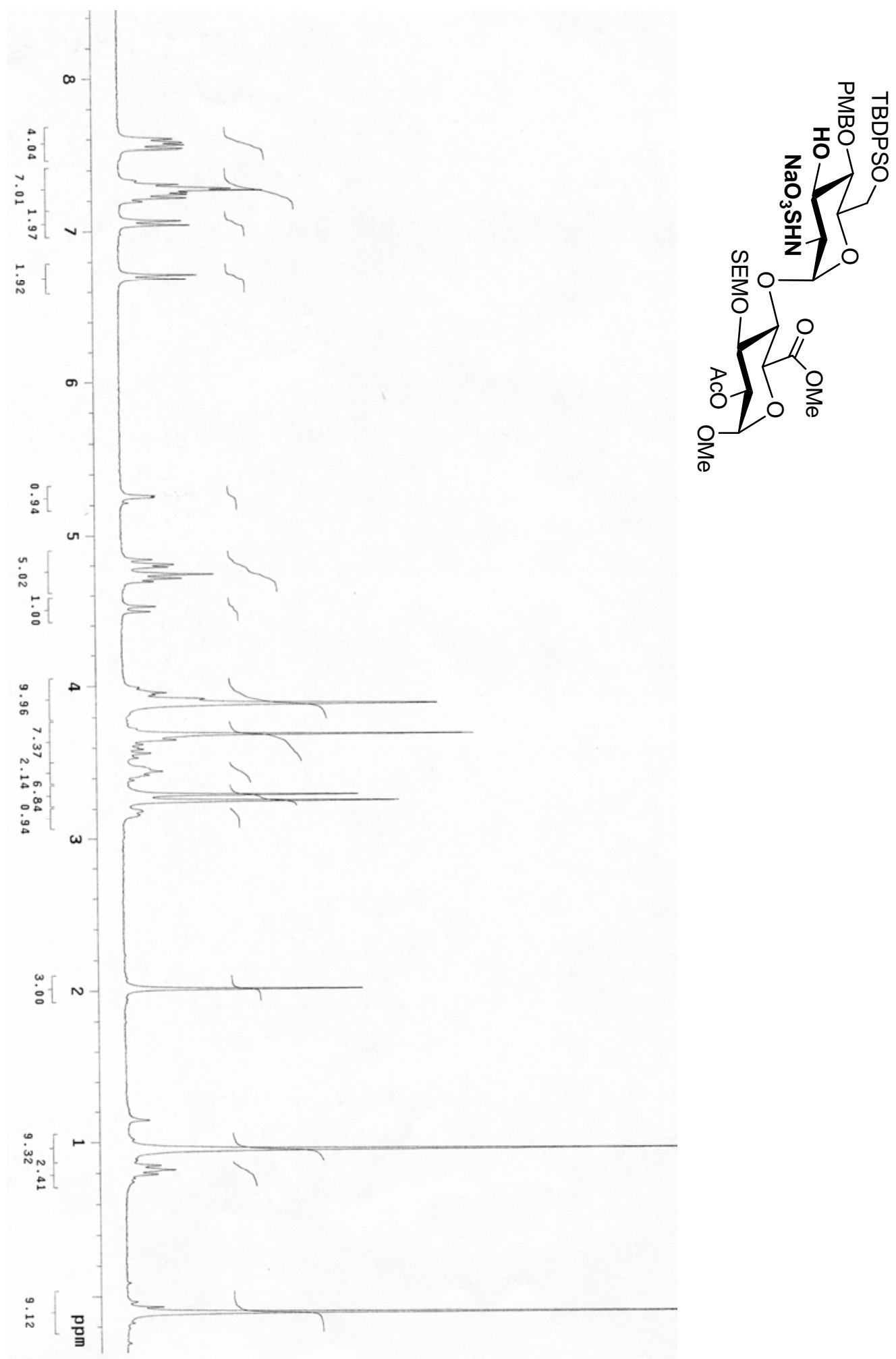

${ }^{1} \mathrm{H}$ NMR (300 MHz, 25\% $\mathrm{CD}_{3} \mathrm{OD}$ in $\mathrm{CDCl}_{3}$ ) Disaccharide 2'-N-Sulfate-3'-OH 19 
Fan, R. H.; Achkar, J.; Hernández-Torres, J. M.; Wei, A.
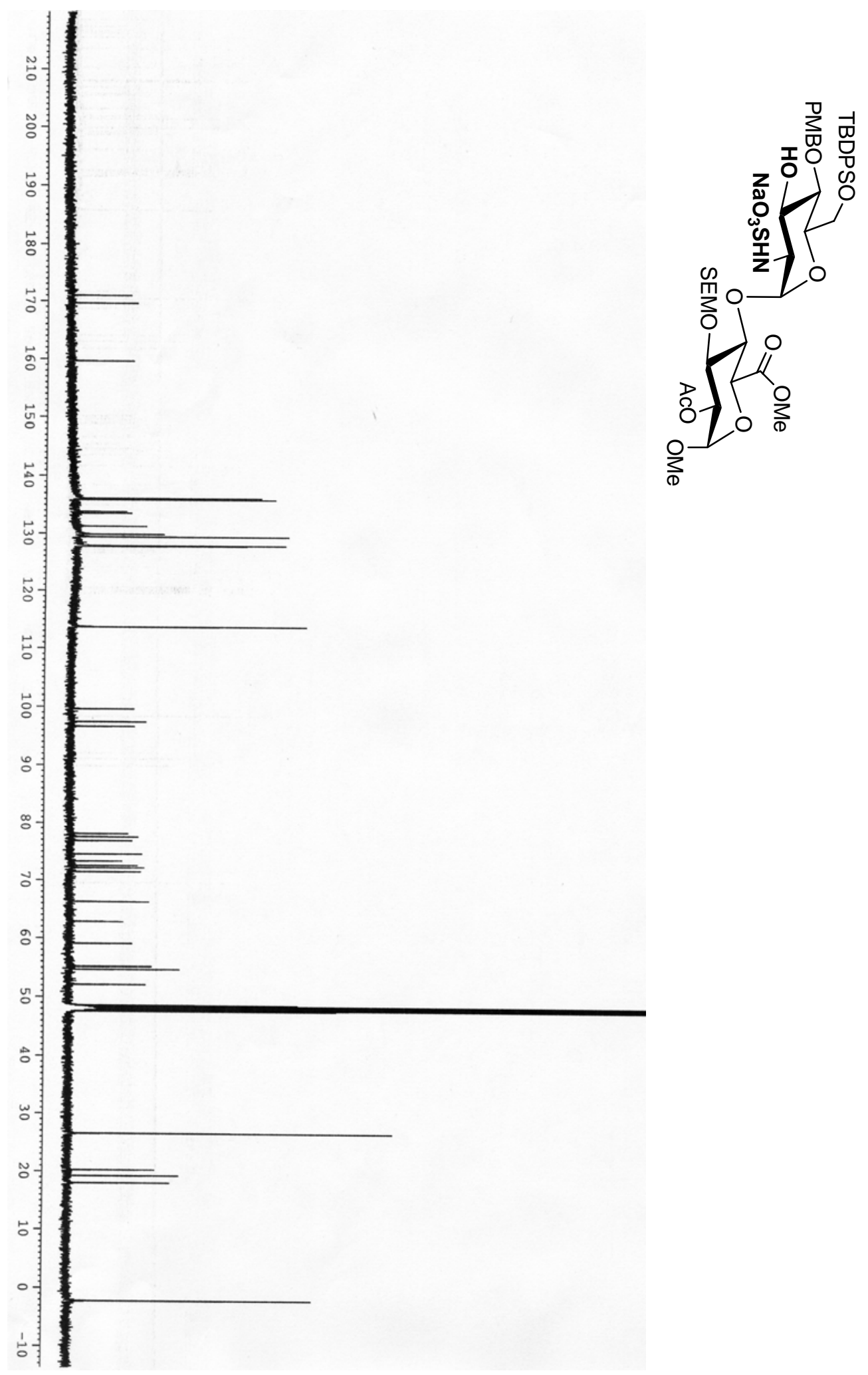

${ }^{13} \mathrm{C}$ NMR (500 MHz, CD 3 OD) Disaccharide 2'-N-Sulfate-3'-OH 19 